\title{
Chemoselective Ketone Synthesis by the Addition of Organometallics to $N$-Acylazetidines
}

\author{
Chengwei Liu, $^{\dagger}$ Marcel Achtenhagen ${ }^{\dagger}$ and Michal Szostak ${ }^{*}{ }^{\dagger}$ \\ 'Department of Chemistry, Rutgers University, 73 Warren Street, Newark, New Jersey 07102, \\ United States \\ michal.szostak@rutgers.edu
}

\section{Supplementary Information}

Table of Contents $\quad 1$

List of Known Compounds/General Methods 2

Experimental Procedures and Characterization Data 3

- General Procedures 3

- Characterization Data of Starting Materials 5

- Characterization Data of Ketone Products 8

- Mechanistic Studies 21

- A) Competition Experiments $\quad 21$

○ B) Selectivity Studies $\quad 22$

$\circ$ C) Effect of Lewis Acid Additives 23

References $\quad 24$

${ }^{1} \mathrm{H}$ and ${ }^{13} \mathrm{C}$ NMR Spectra $\quad 26$

Corresponding Author:

Prof. Dr. M. Szostak

Department of Chemistry, Rutgers University

73 Warren Street, Newark, NJ 07102, United States

E-mail: michal.szostak@rutgers.edu 


\section{List of Known Compounds/General Methods}

All compounds reported in the manuscript have been previously described in the literature or are commercially available unless stated otherwise. ${ }^{1-21}$ Amides were prepared by standard methods. ${ }^{1-}$ ${ }^{7}$ All experiments involving organometallic reagents were performed using standard Schlenk techniques under argon or nitrogen atmosphere unless stated otherwise. All organometallic reagents have been titrated prior to use. ${ }^{22}$ All solvents were purchased at the highest commercial grade and used as received or after purification by passing through activated alumina columns or distillation from sodium/benzophenone under nitrogen. All solvents were deoxygenated prior to use. All other chemicals were purchased at the highest commercial grade and used as received. Reaction glassware was oven-dried at $140{ }^{\circ} \mathrm{C}$ for at least $24 \mathrm{~h}$ or flame-dried prior to use, allowed to cool under vacuum and purged with argon (three cycles). All products were identified using ${ }^{1} \mathrm{H}$ NMR analysis and comparison with authentic samples. GC and/or GC/MS analysis was used for volatile products. All yields refer to yields determined by ${ }^{1} \mathrm{H}$ NMR and/or GC or GC/MS using an internal standard (optimization) and isolated yields (preparative runs) unless stated otherwise. Yields refer to isolated compounds, estimated to be $>95 \%$ pure as determined by ${ }^{1} \mathrm{H}$ NMR and/or GC. ${ }^{1} \mathrm{H}$ NMR and ${ }^{13} \mathrm{C}$ NMR spectra were recorded in $\mathrm{CDCl}_{3}$ on Bruker spectrometers at $500\left({ }^{1} \mathrm{H} \mathrm{NMR}\right)$ and $125 \mathrm{MHz}\left({ }^{13} \mathrm{C}\right.$ NMR). All shifts are reported in parts per million (ppm) relative to residual $\mathrm{CHCl}_{3}$ peak (7.28 and $77.2 \mathrm{ppm},{ }^{1} \mathrm{H}$ NMR and ${ }^{13} \mathrm{C} \mathrm{NMR}$, respectively). All coupling constants (J) are reported in hertz (Hz). Abbreviations are: s, singlet; d, doublet; t, triplet; q, quartet; brs, broad singlet. GC-MS chromatography was performed using Agilent HP6890 GC System and Agilent 5973A inert XL EI/CI MSD using helium as the carrier gas at a flow rate of $1 \mathrm{~mL} / \mathrm{min}$ and an initial oven temperature of $50^{\circ} \mathrm{C}$. The injector temperature was $250{ }^{\circ} \mathrm{C}$. The detector temperature was $250^{\circ} \mathrm{C}$. For runs with the initial oven temperature of $50{ }^{\circ} \mathrm{C}$, temperature was increased with a $10{ }^{\circ} \mathrm{C} / \mathrm{min}$ ramp after $50{ }^{\circ} \mathrm{C}$ hold for $3 \mathrm{~min}$ to a final temperature of $220^{\circ} \mathrm{C}$, then hold at $220^{\circ} \mathrm{C}$ for $15 \mathrm{~min}$ (splitless mode of injection, total run time of $22.00 \mathrm{~min}$ ). High-resolution mass spectra (HRMS) were measured on a 7T Bruker Daltonics FT-MS instrument. All flash chromatography was performed using silica gel, $60 \AA$, 300 mesh. TLC analysis was carried out on glass plates coated with silica gel 60 F254, $0.2 \mathrm{~mm}$ thickness. The plates were visualized using a $254 \mathrm{~nm}$ ultraviolet lamp or aqueous potassium permanganate

solutions. ${ }^{1} \mathrm{H}$ NMR, ${ }^{13} \mathrm{C}$ NMR and MS data are given for all compounds in the Supporting 
Experimental for characterization purposes. ${ }^{1} \mathrm{H}$ NMR, ${ }^{13} \mathrm{C}$ NMR, MS and HRMS data are reported for all new compounds.

\section{Experimental Procedures and Characterization Data}

General Procedure for Amide Synthesis. A round-bottomed flask (25 mL) equipped with a stir bar was charged with potassium carbonate (typically, $5.34 \mathrm{mmol}, 1.2$ equiv), $\mathrm{Et}_{2} \mathrm{O}(3.0 \mathrm{~mL}), \mathrm{H}_{2} \mathrm{O}$ (3.0 mL), and azetidine hydrochloride (typically, $5.34 \mathrm{mmol}, 1.2$ equiv), cooled to $0{ }^{\circ} \mathrm{C}$, and acid chloride (typically, $4.45 \mathrm{mmol}, 1.0$ equiv) was added dropwise with vigorous stirring. The reaction mixture was stirred at $0{ }^{\circ} \mathrm{C}$ for $15 \mathrm{~min}$. After the indicated time, the reaction mixture was extracted with $\mathrm{Et}_{2} \mathrm{O}(2 \times 30 \mathrm{~mL})$, organic layers were combined, washed with $\mathrm{NaOH}(1.0 \mathrm{~N}$, $2 \times 10 \mathrm{~mL}), \mathrm{HCl}(1.0 \mathrm{~N}, 2 \times 10 \mathrm{~mL})$, dried, filtered and concentrated. Unless stated otherwise, the crude product was purified by recrystallization $\left(\mathrm{Et}_{2} \mathrm{O}\right)$ or chromatography on silica gel (EtOAc/hexanes) to give analytically pure product.

General Procedure for Ketone Synthesis from Azetidinyl Amides. An oven-dried vial equipped with a stir bar was charged with an $N$-acylazetidine substrate (typically, $0.50 \mathrm{mmol}, 1.0$ equiv), placed under a positive pressure of nitrogen, and subjected to three evacuation/backfilling cycles. Tetrahydrofuran (typically, $5.0 \mathrm{~mL}, 0.10 \mathrm{M}$ ) was added, the reaction mixture was cooled to $0{ }^{\circ} \mathrm{C}$ or $-78{ }^{\circ} \mathrm{C}$, followed by dropwise addition of an organometallic reagent (typically, 3.0 equiv) with vigorous stirring. After the indicated time, the reaction was quenched by slow addition of $\mathrm{HCl}(1.0 \mathrm{~N}, 5 \mathrm{~mL})$, extracted with $\mathrm{Et}_{2} \mathrm{O}(2 \times 30 \mathrm{~mL})$, organic layers were combined, dried, filtered and concentrated. The sample was analyzed by ${ }^{1} \mathrm{H} \mathrm{NMR}\left(\mathrm{CDCl}_{3}, 500 \mathrm{MHz}\right)$ and GC-MS to obtain selectivity, conversion and yield using internal standard and comparison with authentic samples. Purification by chromatography on silica gel afforded the title product.

Note: For reactions that were run at $-78{ }^{\circ} \mathrm{C}$, the reaction was quenched with $\mathrm{HCl}(1.0 \mathrm{~N}, 5 \mathrm{~mL})$ at $-78{ }^{\circ} \mathrm{C}$ and then allowed to reach room temperature. All other reactions were quenched with $\mathrm{HCl}$ $(1.0 \mathrm{~N}, 5 \mathrm{~mL})$ at room temperature. For the addition of the reactive organometallic reagents, the rate of addition was about 1 drop every 3 seconds ( $\mathrm{MeLi}, n-\mathrm{BuLi}, t$-BuLi). For the less reactive organometallic reagents, the rate of addition was about 1 drop every second. 
Representative Procedure. An oven-dried vial equipped with a stir bar was charged with azetidin-1-yl(phenyl)methanone $(0.080 \mathrm{~g}, 0.50 \mathrm{mmol}, 1.0$ equiv), placed under a positive pressure of nitrogen, and subjected to three evacuation/backfilling cycles. Tetrahydrofuran (5.0 $\mathrm{mL}, 0.10 \mathrm{M})$ was added, the reaction mixture was cooled to $-78{ }^{\circ} \mathrm{C}$, phenyllithium $(1.90 \mathrm{M}$ solution in $\mathrm{Bu}_{2} \mathrm{O}, 0.79 \mathrm{~mL}, 1.50 \mathrm{mmol}, 3.0$ equiv) was added dropwise with vigorous stirring at $78{ }^{\circ} \mathrm{C}$, and the reaction was stirred at $-78{ }^{\circ} \mathrm{C}$ for $15 \mathrm{~min}$. After the indicated time, the reaction mixture was quenched by slow addition of $\mathrm{HCl}(1.0 \mathrm{~N}, 5 \mathrm{~mL})$, extracted with $\mathrm{Et}_{2} \mathrm{O}(2 \times 30 \mathrm{~mL})$, organic layers were combined, dried, filtered and concentrated. The sample was analyzed by ${ }^{1} \mathrm{H}$ $\mathrm{NMR}\left(\mathrm{CDCl}_{3}, 500 \mathrm{MHz}\right)$ and GC-MS to obtain selectivity, conversion and yield using internal standard. Purification by chromatography on silica gel (EtOAc/hexanes) afforded the title product $(76.6 \mathrm{mg})$. Yield $84 \%$. Characterization data are included in the section below. 


\section{Characterization Data for Starting Materials}

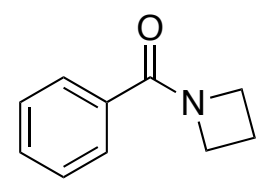

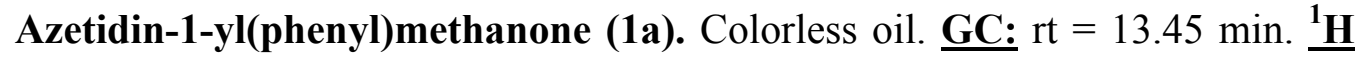
NMR (500 MHz, CDCl $\left.{ }_{3}\right) \delta 7.45-7.44(\mathrm{~d}, J=7.1 \mathrm{~Hz}, 2 \mathrm{H}), 7.26-7.23$ (t, $J=7.3$ $\mathrm{Hz}, 1 \mathrm{H}), 7.21-7.18(\mathrm{t}, J=7.7 \mathrm{~Hz}, 2 \mathrm{H}), 4.07-4.04$ (t, $J=7.5 \mathrm{~Hz}, 2 \mathrm{H}), 4.01-3.97$

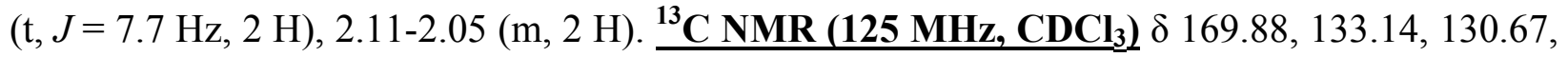
128.15, 127.63, 53.24, 48.72, 15.88. $\underline{\text { MS }}=161.1$ (EI). Spectroscopic data matched literature values. ${ }^{1}$

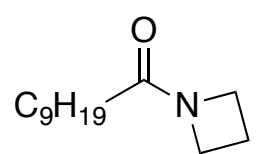

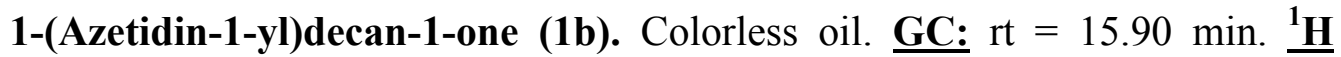
NMR (500 MHz, $\left.\mathbf{C D C l}_{3}\right) \delta 4.12-4.10(\mathrm{~d}, J=6.4 \mathrm{~Hz}, 2 \mathrm{H}), 4.00-3.99(\mathrm{~d}, J=$ $6.7 \mathrm{~Hz}, 2 \mathrm{H}), 2.25-2.23(\mathrm{~d}, J=6.8 \mathrm{~Hz}, 2 \mathrm{H}), 2.04-2.02(\mathrm{~d}, J=6.0 \mathrm{~Hz}, 2 \mathrm{H})$, $\left.1.58(\mathrm{~s}, 2 \mathrm{H}), 1.25(\mathrm{~s}, 12 \mathrm{H}), 0.87-0.86(\mathrm{~d}, J=6.7 \mathrm{~Hz}, 3 \mathrm{H}) . \underline{{ }^{13} \mathbf{C} \text { NMR (125 MHz, CDCl }} 3\right) \delta$ $173.37,50.07,47.69,31.85,31.23,29.44,29.44,29.39,29.26,24.92,22.64,15.00,14.08$. MS $=$ 211.2 (EI). Spectroscopic data matched literature values. ${ }^{2}$

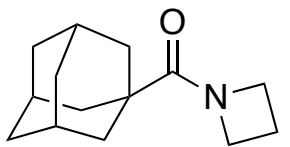

(Adamantan-1-yl)(azetidin-1-yl)methanone (1c). White solid. GC: $\mathrm{rt}=$ 17.82 min. ${ }^{1} \mathbf{H}$ NMR (500 MHz, $\left.\mathbf{C D C l}_{3}\right) \delta 4.43(\mathrm{~s}, 2 \mathrm{H}), 4.02$ (s, $\left.2 \mathrm{H}\right), 2.28-$ $2.22(\mathrm{~m}, 2 \mathrm{H}), 2.02(\mathrm{~s}, 3 \mathrm{H}), 1.92(\mathrm{~s}, 6 \mathrm{H}), 1.75-1.69(\mathrm{t}, J=15.8 \mathrm{~Hz}, 6 \mathrm{H}) . \underline{{ }^{13} \mathrm{C}}$ $\underline{\left.\text { NMR (125 MHz, } \mathbf{C D C l}_{3}\right)} \delta 177.00,53.34,49.23,41.12,38.40,36.62,28.17,16.23 . \underline{\mathbf{M S}}=219.2$ (EI). Spectroscopic data matched literature values. ${ }^{3}$<smiles>COc1ccc(C(=O)N2CCC2)cc1</smiles>

Azetidin-1-yl(4-methoxyphenyl)methanone (1d). Colorless oil. $\underline{\text { GC: }} \mathrm{rt}=$ $16.58 \mathrm{~min} .{ }^{1} \mathbf{H}$ NMR (500 MHz, $\left.\mathbf{C D C l}_{3}\right) \delta 7.63-7.61(\mathrm{~d}, J=8.7 \mathrm{~Hz}, 2 \mathrm{H})$, 6.91-6.89 (d, $J=8.7 \mathrm{~Hz}, 2 \mathrm{H}), 4.32-4.22(\mathrm{~d}, J=54.3 \mathrm{~Hz}, 4 \mathrm{H}), 3.84$ (s, $3 \mathrm{H})$,

2.36-2.30 (m, $2 \mathrm{H}) .{ }^{13} \mathbf{C}$ NMR (125 MHz, CDCl $\left.\underline{3}_{2}\right) \delta 169.88,161.58,129.77,125.60,113.52$, 55.33, 53.59, 48.97, 16.11. $\underline{\mathbf{M S}}=191.1(\mathrm{EI})$. Spectroscopic data matched literature values. ${ }^{1}$<smiles>Cc1ccc(C(=O)N2CCC2)cc1</smiles>

Azetidin-1-yl(p-tolyl)methanone (1e). Light yellow solid. $\underline{\text { GC: }} \mathrm{rt}=14.78$ min. ${ }^{1} \mathbf{H}$ NMR (500 MHz, $\left.\mathbf{C D C l}_{3}\right) \delta 7.56-7.54(\mathrm{~d}, J=8.1 \mathrm{~Hz}, 2 \mathrm{H}), 7.23-$ $7.21(\mathrm{~d}, J=7.9 \mathrm{~Hz}, 2 \mathrm{H}), 4.33-4.31(\mathrm{t}, J=6.6 \mathrm{~Hz}, 2 \mathrm{H}), 4.25-4.22(\mathrm{t}, J=7.0$ 
Hz, $2 \mathrm{H}), 2.40$ (s, $3 \mathrm{H}), 2.38-2.32$ (m, 2 H). ${ }^{13} \mathbf{C}$ NMR (125 MHz, $\left.\mathbf{C D C l}_{3}\right) \delta 170.30,141.13$, 130.45, 128.95, 127.90, 53.44, 48.87, 21.47, 16.08. $\underline{\mathbf{M S}}=175.1$ (EI). Spectroscopic data matched literature values. ${ }^{1}$<smiles>O=C(c1ccc(C(F)(F)F)cc1)N1CCC1</smiles>

Azetidin-1-yl(4-(trifluoromethyl)phenyl)methanone (1f). New compound. White solid. Mp: $91-93{ }^{\circ} \mathrm{C}$. GC: $\mathrm{rt}=12.98 \mathrm{~min} . \underline{{ }^{1} \mathbf{H} \text { NMR (500 } \mathbf{~ M H z}}$ $\underline{\mathbf{C D C l}_{3}} 2 \delta$ 7.76-7.75 (d, $\left.J=8.1 \mathrm{~Hz}, 2 \mathrm{H}\right), 7.70-7.68(\mathrm{~d}, J=8.2 \mathrm{~Hz}, 2 \mathrm{H})$, 4.33-4.30 (t, $J=7.6 \mathrm{~Hz}, 2 \mathrm{H}), 4.28-4.25(\mathrm{t}, J=7.8 \mathrm{~Hz}, 2 \mathrm{H}), 2.42-2.36(\mathrm{~m}, 2 \mathrm{H}) . \underline{{ }^{13} \mathbf{C} \text { NMR (125 }}$ $\underline{\left.\mathbf{M H z}, \mathbf{C D C l}_{3}\right)} \delta 168.77,136.68,132.57\left(\mathrm{q}, J^{2}=32.3 \mathrm{~Hz}\right), 128.20,125.39\left(\mathrm{q}, J^{3}=3.7 \mathrm{~Hz}\right)$,

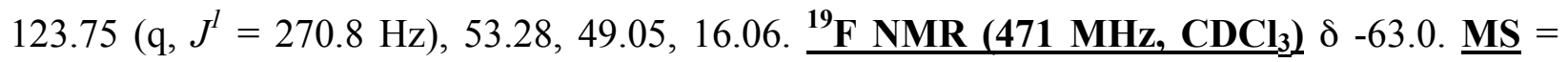
229.1 (EI). $\underline{\text { HRMS }}$ calcd for $\mathrm{C}_{22} \mathrm{H}_{19} \mathrm{O}_{2} \mathrm{~N}_{2} \mathrm{~F}_{6}\left(2 \mathrm{M}^{+}-\mathrm{H}\right) 457.1345$, found 457.1325 .<smiles>O=C(c1ccc(F)cc1)N1CCC1</smiles>

Azetidin-1-yl(4-fluorophenyl)methanone (1g). White solid. $\underline{\text { GC: }} \mathbf{r t}=13.16$ $\min .{ }^{1}$ H NMR (500 MHz, $\left.\mathbf{C D C l}_{3}\right) \delta$ 7.68-7.65 (m, $\left.2 \mathrm{H}\right), 7.12-7.08$ (t, $J=8.6$ Hz, $2 \mathrm{H}), 4.32-4.24$ (d, $J=39.8 \mathrm{~Hz}, 4 \mathrm{H}), 2.40-2.34$ (m, $2 \mathrm{H}) . \underline{{ }^{13} \mathbf{C} \text { NMR (125 }}$ $\left.\underline{\text { MHz }, \mathbf{C D C l}_{3}}\right) \delta 169.13,164.16\left(\mathrm{~d}, J^{l}=249.6 \mathrm{~Hz}\right), 130.16\left(\mathrm{~d}, J^{3}=8.7 \mathrm{~Hz}\right), 129.42\left(\mathrm{~d}, J^{4}=3.2\right.$ $\left.\mathrm{Hz}), 115.37\left(\mathrm{~d}, J^{2}=21.6 \mathrm{~Hz}\right), 53.47,48.97,16.04 . \underline{{ }^{19} \mathbf{F ~ N M R}(471 \mathbf{M H z}, \mathbf{C D C l}} 3\right) \delta-108.8 . \underline{\mathbf{M S}}=$ 179.1 (EI). Spectroscopic data matched literature values. ${ }^{4}$<smiles>O=C(c1ccc(Cl)cc1)N1CCC1</smiles>

Azetidin-1-yl(4-chlorophenyl)methanone (1h). White solid. GC: $\mathrm{rt}=$ 15.46 min. ${ }^{1} \mathbf{H}$ NMR (500 MHz, $\left.\mathbf{C D C l}_{3}\right) \delta 87.61-7.59(\mathrm{~d}, J=8.5 \mathrm{~Hz}, 2 \mathrm{H})$, 7.41-7.39 (d, $J=8.5 \mathrm{~Hz}, 2 \mathrm{H}), 4.33-4.30$ (t, $J=6.9 \mathrm{~Hz}, 2 \mathrm{H}), 4.26-4.23$ (t, $J$ $=7.1 \mathrm{~Hz}, 2 \mathrm{H}), 2.40-2.34(\mathrm{~m}, 2 \mathrm{H}) .{ }^{13} \mathbf{C}$ NMR (125 MHz, $\left.\mathbf{C D C l}_{3}\right) \delta 169.07,136.98,131.67$, 129.30, 128.61, 53.42, 49.00, 16.07. $\underline{\mathbf{M S}}=195.1$ (EI). Spectroscopic data matched literature

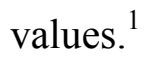<smiles>O=C(c1ccc(Br)cc1)N1CCC1</smiles>
Azetidin-1-yl(4-bromophenyl)methanone (1i). White solid. GC: $\mathrm{rt}=$ 16.55 min. ${ }^{1} \mathbf{H}$ NMR (500 MHz, $\left.\mathbf{C D C l}_{3}\right) \delta$ 7.57-7.55 (d, $\left.J=8.4 \mathrm{~Hz}, 2 \mathrm{H}\right)$, 7.53-7.51 (d, $J=8.3 \mathrm{~Hz}, 2 \mathrm{H}), 4.31-4.23(\mathrm{~d}, J=35.9 \mathrm{~Hz}, 4 \mathrm{H}), 2.40-2.34$ (m, 2 H). ${ }^{13} \mathbf{C}$ NMR (125 MHz, $\left.\mathbf{C D C l}_{3}\right) \delta 169.16,132.13,131.59,129.49,125.42,53.41,49.01$, 16.07. $\underline{\mathrm{MS}}=239.0$ (EI). Spectroscopic data matched literature values. ${ }^{5}$ 
<smiles>COc1ccc(/C=C/C(=O)N2CCC2)cc1</smiles>
(E)-1-(Azetidin-1-yl)-3-(4-methoxyphenyl)prop-2-en-1-one (1j). New compound. White solid. Mp: $138-139^{\circ} \mathrm{C}$. GC: $\mathrm{rt}=20.06 \mathrm{~min}$. $\underline{\left.{ }^{1} \text { H NMR (500 MHz, } \mathbf{C D C l}_{3}\right)} \delta$ 7.63-7.60 (d, $\left.J=15.6 \mathrm{~Hz}, 1 \mathrm{H}\right)$, 7.50-7.48 (d, $J=8.5 \mathrm{~Hz}, 2 \mathrm{H}), 6.92-6.90(\mathrm{~d}, J=8.5 \mathrm{~Hz}, 2 \mathrm{H}), 6.36-6.33(\mathrm{~d}, J=15.6 \mathrm{~Hz}, 1 \mathrm{H})$, 4.33-4.30 (t, $J=7.4 \mathrm{~Hz}, 2 \mathrm{H}), 4.17-4.14$ (t, $J=7.6 \mathrm{~Hz}, 2 \mathrm{H}), 3.85$ (s, $3 \mathrm{H}), 2.38-2.32$ (m, $2 \mathrm{H})$. ${ }^{13} \mathbf{C}$ NMR (125 MHz, $\left.\mathbf{C D C l}_{3}\right) \delta 166.38,160.89,140.87,129.44,127.81,114.20,113.19,55.36$, 50.02, 48.03, 15.36. $\underline{\text { MS }}=217.1(\mathrm{EI})$. $\underline{\text { HRMS }}$ calcd for $\mathrm{C}_{13} \mathrm{H}_{16} \mathrm{O}_{2} \mathrm{~N}\left(\mathrm{M}^{+}+\mathrm{H}\right) 218.1176$, found 218.1175 .<smiles>CON(C)C(=O)c1ccccc1</smiles>

$N$-Methoxy- $N$-methylbenzamide (1k). Colorless oil. $\underline{\text { GC: }} \mathrm{rt}=10.34 \mathrm{~min} . \underline{\mathbf{H}}$ $\underline{\left.\text { NMR (500 MHz, } \mathbf{C D C l}_{3}\right)} \delta$ 7.70-7.68 (d, $\left.J=7.7 \mathrm{~Hz}, 2 \mathrm{H}\right), 7.49-7.46(\mathrm{t}, J=$ $6.5 \mathrm{~Hz}, 1 \mathrm{H}), 7.43-7.40(\mathrm{t}, J=7.0 \mathrm{~Hz}, 2 \mathrm{H}), 3.57(\mathrm{~s}, 3 \mathrm{H}), 3.38(\mathrm{~s}, 3 \mathrm{H}) . \underline{\mathbf{M S}}=$ 165.1 (EI). ${ }^{13} \mathbf{C}$ NMR (125 MHz, $\left.\mathbf{C D C l}_{3}\right) \delta 169.96,134.14,130.55,128.14,128.01,61.04$, 33.77. $\underline{\text { MS }}=165.1(\mathrm{EI})$. Spectroscopic data matched literature values. ${ }^{6}$

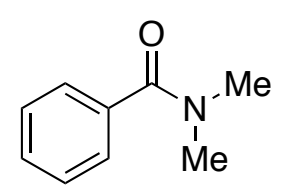

$\boldsymbol{N}, \mathbf{N}$-Dimethylbenzamide (11). Colorless oil. GC: $\mathrm{rt}=10.50 \mathrm{~min} . \underline{{ }^{1} \mathbf{H} \text { NMR }}$ $\underline{\left.\mathbf{5 0 0} \mathbf{M H z}, \mathbf{C D C l}_{3}\right)} \delta 7.42(\mathrm{~m}, 5 \mathrm{H}), 3.14(\mathrm{~s}, 3 \mathrm{H}), 3.00(\mathrm{~s}, 3 \mathrm{H}) . \underline{{ }^{13} \mathbf{C ~ N M R}}$ $\underline{\left(125 \mathbf{M H z}_{2} \mathbf{C D C l}_{3}\right)} \delta 171.64,136.36,129.50,128.34,127.05,39.58,35.35$. $\underline{\mathbf{M S}}=149.1(\mathrm{EI})$. Spectroscopic data matched literature values. ${ }^{7}$<smiles>CC1CN1C(=O)c1ccccc1</smiles>

(2-Methylaziridin-1-yl)(phenyl)methanone (1m). Colorless oil. GC: $\mathrm{rt}=$ $10.32 \min . \underline{\left.{ }^{1} \mathbf{H} \text { NMR (500 MHz, } \mathbf{C D C l}_{3}\right)} \delta 8.05-8.04(\mathrm{~d}, J=7.7 \mathrm{~Hz}, 2 \mathrm{H})$, 7.58-7.55 (t, $J=6.9 \mathrm{~Hz}, 1 \mathrm{H}), 7.49-7.46(\mathrm{t}, J=7.3 \mathrm{~Hz}, 2 \mathrm{H}), 2.62-2.56(\mathrm{~m}$, $2 \mathrm{H}), 2.17-2.16(\mathrm{~d}, J=3.1 \mathrm{~Hz}, 1 \mathrm{H}), 1.42-1.41(\mathrm{~d}, J=5.3 \mathrm{~Hz}, 3 \mathrm{H}) .{ }^{{ }^{13} \mathbf{C} \text { NMR }(\mathbf{1 2 5} \mathbf{M H z}}$ $\underline{\mathbf{C D C l}_{3}} \underline{\underline{ }} \delta 179.28,133.54,132.62,129.04,128.40,34.61,32.14,17.79 . \underline{\mathrm{MS}}=161.1$ (EI). Spectroscopic data matched literature values. ${ }^{6}$ 


\section{Synthesis of Ketones by Addition of Organometallic Reagents to $\mathrm{N}$-Acylazetidines: Variation of Organometallic Reagents and $\mathrm{N}$-Acylazetidines}

Acetophenone (Scheme 1, Entry 1)

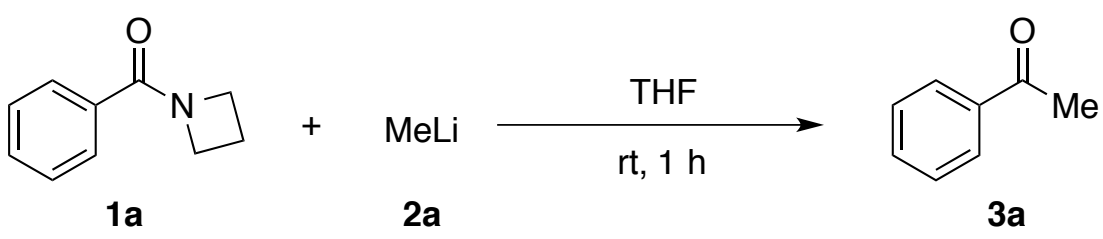

According to the general procedure, the reaction of azetidin-1-yl(phenyl)methanone $(0.50 \mathrm{mmol}$, $0.080 \mathrm{~g}, 1.00$ equiv), methyllithium (1.08 $\mathrm{M}$ solution in $\mathrm{Et}_{2} \mathrm{O}, 1.40 \mathrm{~mL}, 1.50 \mathrm{mmol}, 3.0$ equiv) in THF $(5.0 \mathrm{~mL})$ for $1 \mathrm{~h}$ from $0{ }^{\circ} \mathrm{C}$ to room temperature, afforded after work-up with $\mathrm{HCl}(1.0 \mathrm{~N}, 5$ $\mathrm{mL})$ and chromatography the title compound in $83 \%$ yield $(49.9 \mathrm{mg})$. Colorless oil. $\mathbf{G C}$ : $\mathrm{rt}=$ 8.08 min. ${ }^{1}$ H NMR (500 MHz, CDCl $\left.{ }_{3}\right) \delta 7.99-7.98(\mathrm{~d}, J=7.2 \mathrm{~Hz}, 2 \mathrm{H}), 7.60-7.58(\mathrm{t}, J=7.5 \mathrm{~Hz}$, $1 \mathrm{H}), 7.50-7.47(\mathrm{t}, J=7.9 \mathrm{~Hz}, 2 \mathrm{H}), 2.63(\mathrm{~s}, 3 \mathrm{H}) .{ }^{13} \mathbf{C} \mathbf{N M R}\left(\mathbf{1 2 5} \mathbf{M H z}, \mathbf{C D C l}_{3}\right) \delta 198.15$, $137.14,133.05,128.57,128.31,26.63 . \underline{\mathbf{M S}}=120.1(\mathrm{EI})$. Spectroscopic data matched literature values. ${ }^{8}$

1-Phenylpentan-1-one (Scheme 1, Entry 2)<smiles>CCCCCCCCCCCCCCCCCCCC(=O)c1ccccc1</smiles>

According to the general procedure, the reaction of azetidin-1-yl(phenyl)methanone $(0.50 \mathrm{mmol}$, $0.080 \mathrm{~g}, 1.00$ equiv), $n$-butyllithium (1.36 M solution in hexanes, $0.55 \mathrm{~mL}, 0.75 \mathrm{mmol}, 1.5$ equiv) in $\mathrm{THF}(5.0 \mathrm{~mL})$ for $15 \mathrm{~min}$ at $-78^{\circ} \mathrm{C}$, afforded after work-up with $\mathrm{HCl}(1.0 \mathrm{~N}, 5 \mathrm{~mL})$ and chromatography the title compound in $82 \%$ yield $(66.6 \mathrm{mg})$. Yellow oil. GC: $\mathrm{rt}=11.19 \mathrm{~min} .{ }^{1} \underline{\mathbf{H}}$ $\underline{\left.\text { NMR (500 MHz, } \mathbf{C D C l}_{3}\right)} \delta$ 7.99-7.97 (d, $\left.J=7.2 \mathrm{~Hz}, 2 \mathrm{H}\right), 7.58-7.55$ (t, $\left.J=7.5 \mathrm{~Hz}, 1 \mathrm{H}\right), 7.48$ 7.45 (t, $J=7.9 \mathrm{~Hz}, 2 \mathrm{H}), 3.00-2.97$ (t, $J=7.5 \mathrm{~Hz}, 2 \mathrm{H}), 1.77-1.71$ (m, $2 \mathrm{H}), 1.45-1.40$ (m, $2 \mathrm{H})$, 0.99-0.96 (t, $J=7.4 \mathrm{~Hz}, 3 \mathrm{H}) .{ }^{13} \mathbf{C}$ NMR (125 MHz, CDCl 3 ) $\delta 200.54,137.11,132.86,128.55$, 128.06, 38.33, 26.49, 22.50, 13.96. $\underline{\mathrm{MS}}=162.1$ (EI). Spectroscopic data matched literature values. $^{8}$ 
2,2-Dimethyl-1-phenylpropan-1-one (Scheme 1, Entry 3)<smiles>CC(C)(C)C(=O)c1ccccc1</smiles>

According to the general procedure, the reaction of azetidin-1-yl(phenyl)methanone $(0.50 \mathrm{mmol}$, $0.080 \mathrm{~g}, 1.00$ equiv), tert-butyllithium $(0.78 \mathrm{M}$ solution in pentane, $1.92 \mathrm{~mL}, 1.50 \mathrm{mmol}, 3.0$ equiv) in THF $(5.0 \mathrm{~mL})$ for $1 \mathrm{~h}$ from $0{ }^{\circ} \mathrm{C}$ to room temperature, afforded after work-up with $\mathrm{HCl}$ $(1.0 \mathrm{~N}, 5 \mathrm{~mL})$ and chromatography the title compound in $83 \%$ yield $(67.4 \mathrm{mg})$. Yellow oil. GC: $\mathrm{rt}=8.64 \mathrm{~min} . \underline{{ }^{1} \mathbf{H} \text { NMR }\left(\mathbf{5 0 0} \mathbf{~ M H z}, \mathbf{C D C l}_{3}\right.} \delta \delta 7.72-7.70(\mathrm{~d}, J=7.2 \mathrm{~Hz}, 2 \mathrm{H}), 7.49-7.47(\mathrm{t}, J=7.5$ $\mathrm{Hz}, 1 \mathrm{H}), 7.43-7.41$ (t, $J=7.6 \mathrm{~Hz}, 2 \mathrm{H}), 1.38$ (s, 9 H) ${ }^{13} \mathbf{C}$ NMR (125 MHz, $\mathbf{C D C l}_{3}$ ) $\delta 209.27$, 138.61, 130.79, 128.04, 127.82, 44.20, 28.02. $\underline{\mathbf{M S}}=162.1$ (EI). Spectroscopic data matched literature values. ${ }^{9}$

Benzophenone (Scheme 1, Entry 4)

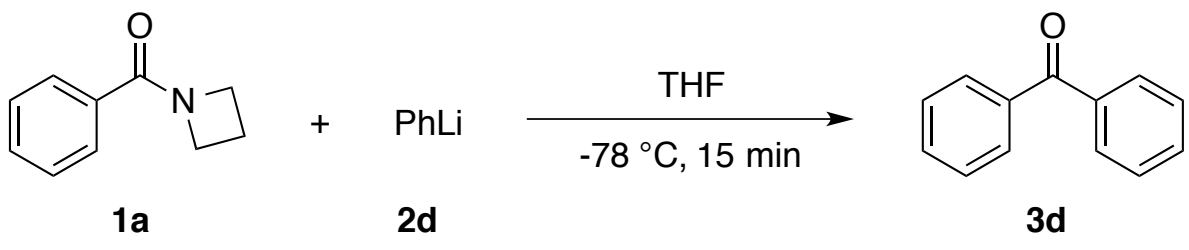

According to the general procedure, the reaction of azetidin-1-yl(phenyl)methanone $(0.50 \mathrm{mmol}$, $0.080 \mathrm{~g}, 1.00$ equiv), phenyllithium ( $1.90 \mathrm{M}$ solution in $\mathrm{Bu}_{2} \mathrm{O}, 0.79 \mathrm{~mL}, 1.50 \mathrm{mmol}, 3.0$ equiv) in THF $(5.0 \mathrm{~mL})$ for $15 \mathrm{~min}$ at $-78{ }^{\circ} \mathrm{C}$, afforded after work-up with $\mathrm{HCl}(1.0 \mathrm{~N}, 5 \mathrm{~mL})$ and chromatography the title compound in $84 \%$ yield $(76.6 \mathrm{mg})$. Light yellow oil. $\underline{\mathbf{G C}}$ : $\mathrm{rt}=13.75$ $\min . \underline{{ }^{1} \text { H NMR }\left(\mathbf{5 0 0 ~} \mathbf{~ M H z}, \mathbf{C D C l}_{3}\right)} \delta$ 7.84-7.83 (d, $\left.J=7.1 \mathrm{~Hz}, 4 \mathrm{H}\right), 7.63-7.60(\mathrm{t}, J=7.5 \mathrm{~Hz}, 2$ H), 7.52-7.49 (t, $J=7.8 \mathrm{~Hz}, 4 \mathrm{H}) .{ }^{13} \mathbf{C}$ NMR (125 MHz, CDCl 3 ) $\delta 196.77,137.62,132.44$, $130.08,128.30 . \underline{\mathbf{M S}}=182.1(\mathrm{EI})$. Spectroscopic data matched literature values. ${ }^{10}$

\section{1,3-Diphenylprop-2-yn-1-one (Scheme 1, Entry 5)}<smiles>O=C(c1ccccc1)N1CCC1</smiles>

$1 a$<smiles>[CH]C#Cc1ccccc1</smiles>

$2 e$

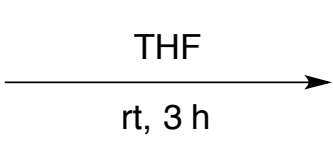

SI-9<smiles>O=C(C#Cc1ccccc1)c1ccccc1</smiles>

$3 e$ 
According to the general procedure, the reaction of azetidin-1-yl(phenyl)methanone $(0.50 \mathrm{mmol}$, $0.080 \mathrm{~g}, 1.00$ equiv), (phenylethynyl)lithium (1.06 M solution in THF, $1.41 \mathrm{~mL}, 1.50 \mathrm{mmol}, 3.0$ equiv) in THF $(5.0 \mathrm{~mL})$ for $3 \mathrm{~h}$ from $0{ }^{\circ} \mathrm{C}$ to room temperature, afforded after work-up with $\mathrm{HCl}$ $(1.0 \mathrm{~N}, 5 \mathrm{~mL})$ and chromatography the title compound in $81 \%$ yield $(83.6 \mathrm{mg})$. Yellow oil. GC: $\mathrm{rt}=17.15$ min. ${ }^{1} \mathbf{H}$ NMR (500 MHz, $\left.\mathbf{C D C l}_{3}\right) \delta 8.27-8.25(\mathrm{~d}, J=7.9 \mathrm{~Hz}, 2 \mathrm{H}), 7.73-7.71(\mathrm{~d}, J=$ $7.8 \mathrm{~Hz}, 2 \mathrm{H}), 7.68-7.65(\mathrm{t}, J=7.5 \mathrm{~Hz}, 1 \mathrm{H}), 7.57-7.53$ (t, $J=7.3 \mathrm{~Hz}, 2 \mathrm{H}), 7.52-7.50$ (d, $J=6.9$

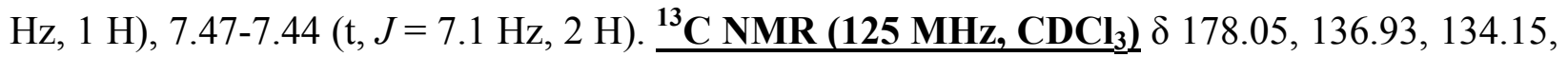
133.10, 130.83, 129.61, 128.72, 128.66, 120.17, 93.13, 86.92. $\underline{\mathbf{M S}}=206.1$ (EI). Spectroscopic data matched literature values. ${ }^{11}$

Phenyl(thiophen-2-yl)methanone (Scheme 1, Entry 6)

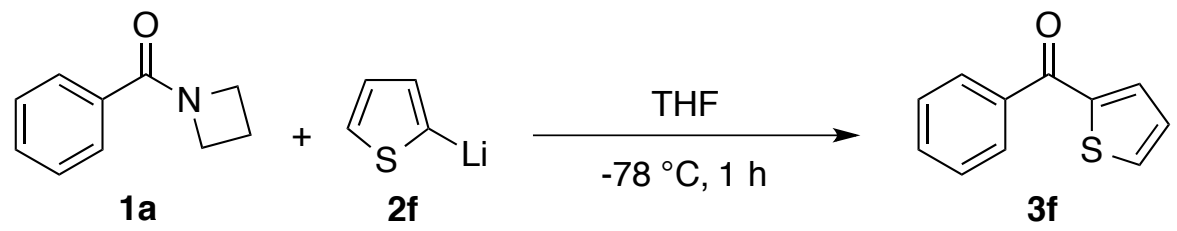

According to the general procedure, the reaction of 2-thienyllithium (prepared from thiophene (1.25 mmol, $0.105 \mathrm{~g}, 2.50$ equiv), $n$-butyllithium (1.36 M solution in hexanes, $0.74 \mathrm{~mL}, 1.00$ mmol, 2.0 equiv) in THF $(2.0 \mathrm{~mL})$ for $1 \mathrm{~h}$ at $\left.-78{ }^{\circ} \mathrm{C}\right)$, azetidin-1-yl(phenyl)methanone $(0.50$ mmol, $0.080 \mathrm{~g}, 1.00 \mathrm{equiv})$ and THF $(3.0 \mathrm{~mL})$ for $1 \mathrm{~h}$ at $-78^{\circ} \mathrm{C}$, afforded after work-up with $\mathrm{HCl}$ $(1.0 \mathrm{~N}, 5 \mathrm{~mL})$ and chromatography the title compound in $86 \%$ yield $(81.0 \mathrm{mg})$. Colorless oil. $\underline{\text { GC: }} \mathrm{rt}=14.34 \mathrm{~min} . \underline{\left.{ }^{1} \mathbf{H} \text { NMR (500 } \mathbf{~ M H z}, \mathbf{C D C l}_{3}\right)} \delta$ 7.90-7.89 (d, $\left.J=7.0 \mathrm{~Hz}, 2 \mathrm{H}\right), 7.75-7.74$ (m, $1 \mathrm{H}), 7.68-7.67(\mathrm{~m}, 1 \mathrm{H}), 7.63-7.60(\mathrm{t}, J=7.4 \mathrm{~Hz}, 1 \mathrm{H}), 7.54-7.51(\mathrm{t}, J=7.8 \mathrm{~Hz}, 2 \mathrm{H}), 7.20-7.18$ $(\mathrm{t}, J=3.9 \mathrm{~Hz}, 1 \mathrm{H}) .{ }^{13} \mathbf{C}$ NMR (125 MHz, $\left.\mathbf{C D C l}_{3}\right) \delta 188.24,143.65,138.16,134.86,134.22$, 132.28, 129.18, 128.43, 127.97. $\underline{\text { MS }}=188.0(\mathrm{EI})$. Spectroscopic data matched literature values. ${ }^{10}$

Furan-2-yl(phenyl)methanone (Scheme 1, Entry 7)

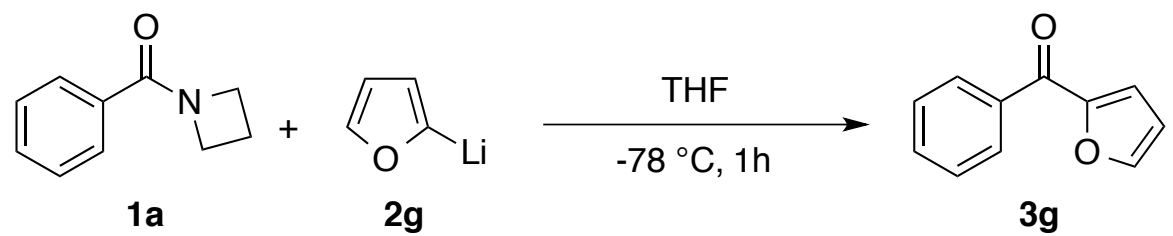

According to the general procedure, the reaction of 2-furyllithium (prepared from furan (1.25 mmol, $0.085 \mathrm{~g}, 2.50$ equiv), $n$-butyllithium (1.36 M solution in hexanes $0.74 \mathrm{~mL}, 1.00 \mathrm{mmol}, 2.0$ 
equiv) in THF $(2.0 \mathrm{~mL})$ for $1 \mathrm{~h}$ at $\left.-78^{\circ} \mathrm{C}\right)$, azetidin-1-yl(phenyl)methanone $(0.50 \mathrm{mmol}, 0.080 \mathrm{~g}$, 1.00 equiv) and THF $(3.0 \mathrm{~mL})$ for $1 \mathrm{~h}$ at $-78^{\circ} \mathrm{C}$, afforded after work-up with $\mathrm{HCl}(1.0 \mathrm{~N}, 5 \mathrm{~mL})$ and chromatography the title compound in $73 \%$ yield $(62.9 \mathrm{mg})$. Yellow oil. $\mathbf{G C}$ : $\mathrm{rt}=12.41 \mathrm{~min}$. $\underline{{ }^{1} \mathbf{H} \text { NMR (500 MHz, } \mathbf{C D C l}_{3}}$ ) $\delta$ 7.98-7.97 (d, $\left.J=7.2 \mathrm{~Hz}, 2 \mathrm{H}\right), 7.71-7.71(\mathrm{~d}, J=0.9 \mathrm{~Hz}, 1 \mathrm{H})$, 7.61-7.58 (t, $J=7.5 \mathrm{~Hz}, 1 \mathrm{H}), 7.51-7.48(\mathrm{t}, J=7.8 \mathrm{~Hz}, 2 \mathrm{H}), 7.24-7.23(\mathrm{~d}, J=3.6 \mathrm{~Hz}, 1 \mathrm{H})$, 6.60-6.59 (m, $1 \mathrm{H}) .{ }^{13} \mathbf{C}$ NMR (125 MHz, $\left.\mathbf{C D C l}_{3}\right) \delta 182.53,152.27,147.12,137.26,132.58$, $129.27,128.42,120.59,112.23 . \underline{\mathbf{M S}}=172.1(\mathrm{EI})$. Spectroscopic data matched literature values. ${ }^{12}$

Acetophenone (Scheme 1, Entry 8)

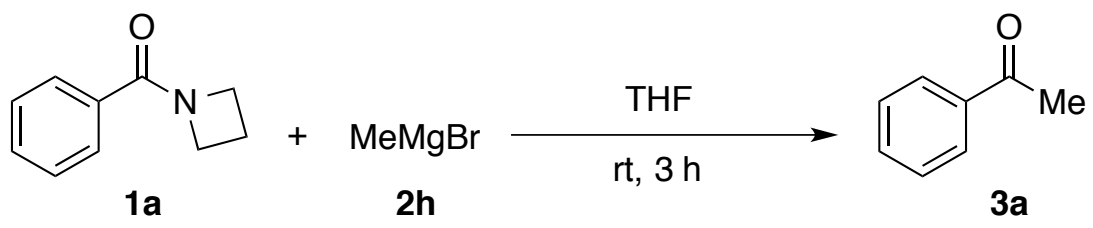

According to the general procedure, the reaction of azetidin-1-yl(phenyl)methanone $(0.50 \mathrm{mmol}$, $0.080 \mathrm{~g}, 1.00$ equiv), methylmagnesium bromide (3.07 $\mathrm{M}$ solution in $\mathrm{Et}_{2} \mathrm{O}, 0.49 \mathrm{~mL}, 1.50 \mathrm{mmol}$, 3.0 equiv) in THF $(5.0 \mathrm{~mL})$ for $3 \mathrm{~h}$ at room temperature, afforded after work-up with $\mathrm{HCl}(1.0 \mathrm{~N}$, $5 \mathrm{~mL})$ and chromatography the title compound in $75 \%$ yield $(45.1 \mathrm{mg})$. Colorless oil. $\mathbf{\text { GC}}$ : $\mathrm{rt}=$ 8.08 min. ${ }^{1}$ H NMR (500 MHz, $\left.\mathbf{C D C l}_{3}\right) \delta$ 7.99-7.98 (d, $\left.J=7.2 \mathrm{~Hz}, 2 \mathrm{H}\right), 7.60-7.58$ (t, $J=7.5 \mathrm{~Hz}$, $1 \mathrm{H}), 7.50-7.47$ (t, $J=7.9 \mathrm{~Hz}, 2 \mathrm{H}), 2.63$ (s, $3 \mathrm{H}) .{ }^{13} \mathbf{C}$ NMR $\left(\mathbf{1 2 5} \mathbf{~ M H z}, \mathbf{C D C l}_{3}\right) \delta 198.15$, 137.14, 133.05, 128.57, 128.31, 26.63. $\underline{\mathbf{M S}}=120.1$ (EI). Spectroscopic data matched literature values. $^{8}$

(4-Methoxyphenyl)(phenyl)methanone (Scheme 1, Entry 9)

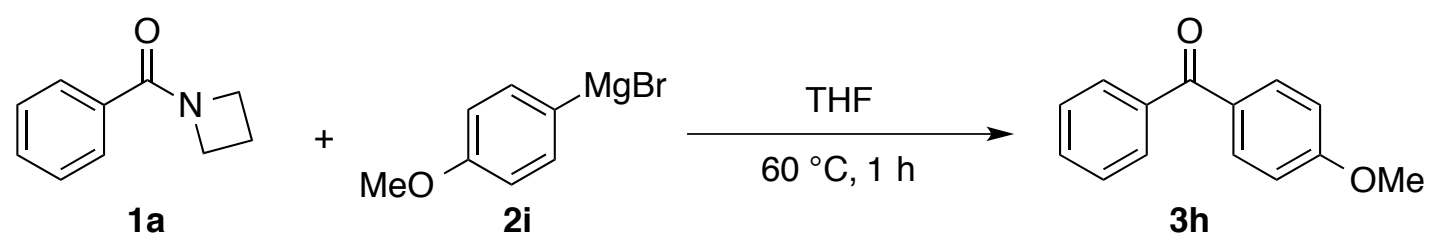

According to the general procedure, the reaction of azetidin-1-yl(phenyl)methanone $(0.50 \mathrm{mmol}$, $0.080 \mathrm{~g}, 1.00$ equiv), (4-methoxyphenyl)magnesium bromide (0.236 M solution in THF, 4.24 $\mathrm{mL}, 1.00 \mathrm{mmol}, 2.0$ equiv) in THF $(3.0 \mathrm{~mL})$ for $1 \mathrm{~h}$ from $0{ }^{\circ} \mathrm{C}$ to $60^{\circ} \mathrm{C}$, afforded after work-up with $\mathrm{HCl}(1.0 \mathrm{~N}, 5 \mathrm{~mL})$ and chromatography the title compound in $94 \%$ yield $(99.8 \mathrm{mg})$. Colorless oil. GC: $\mathrm{rt}=17.02 \mathrm{~min} . \underline{{ }^{1} \mathbf{H} \text { NMR }\left(\mathbf{5 0 0} \mathbf{~ M H z}, \mathbf{C D C l}_{3}\right)} \delta 7.85-7.83(\mathrm{~d}, J=8.9 \mathrm{~Hz}, 2 \mathrm{H})$, 
7.78-7.76 (d, $J=7.1 \mathrm{~Hz}, 2 \mathrm{H}), 7.58-7.56$ (t, $J=7.4 \mathrm{~Hz}, 1 \mathrm{H}), 7.49-7.46$ (t, $J=7.8 \mathrm{~Hz}, 2 \mathrm{H}), 6.98-$ $6.96(\mathrm{~d}, J=8.9 \mathrm{~Hz}, 2 \mathrm{H}), 3.88$ (s, $3 \mathrm{H}) .{ }^{13} \mathbf{C}$ NMR (125 MHz, CDCl 32$) \delta 195.50,163.24,138.30$, 132.54, 131.89, 130.14, 129.71, 128.20, 113.58, 55.49. $\underline{\mathbf{M S}}=212.1$ (EI). Spectroscopic data matched literature values. ${ }^{8}$

Undecan-2-one (Scheme 1, Entry 10)

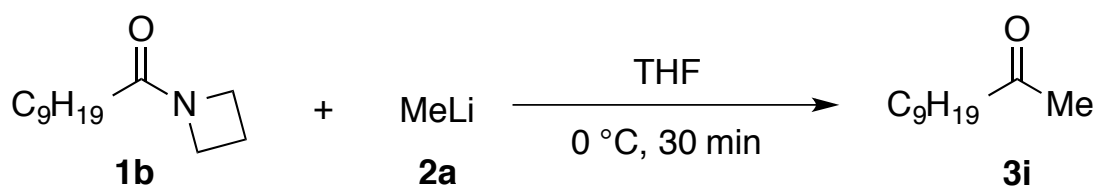

According to the general procedure, the reaction of 1-(azetidin-1-yl)decan-1-one $(0.50 \mathrm{mmol}$, $0.106 \mathrm{~g}, 1.00$ equiv), methyllithium (1.08 $\mathrm{M}$ solution in $\mathrm{Et}_{2} \mathrm{O}, 1.40 \mathrm{~mL}, 1.50 \mathrm{mmol}, 3.0$ equiv) in THF $(5.0 \mathrm{~mL})$ for $30 \mathrm{~min}$ at $0{ }^{\circ} \mathrm{C}$, afforded after work-up with $\mathrm{HCl}(1.0 \mathrm{~N}, 5 \mathrm{~mL})$ and chromatography the title compound in 76\% yield $(64.8 \mathrm{mg})$. Colorless oil. $\underline{\text { GC: }} \mathrm{rt}=9.41 \mathrm{~min} . \underline{{ }^{1} \mathbf{H}}$ NMR (500 MHz, CDCl $\left.\underline{3}_{2}\right) \delta 2.44-2.41(\mathrm{t}, J=7.5 \mathrm{~Hz}, 2 \mathrm{H}), 2.14(\mathrm{~s}, 3 \mathrm{H}), 1.59-1.56(\mathrm{t}, J=7.1 \mathrm{~Hz}$, $2 \mathrm{H}), 1.28-1.27(\mathrm{~m}, 12 \mathrm{H}), 0.90-0.87(\mathrm{t}, J=6.7 \mathrm{~Hz}, 3 \mathrm{H}) .{ }^{13} \mathbf{C}$ NMR (125 MHz, CDCl $32 \delta$

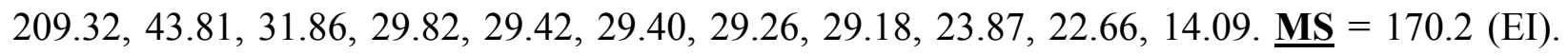
Spectroscopic data matched literature values. ${ }^{13}$

Tetradecan-5-one (Scheme 1, Entry 11)

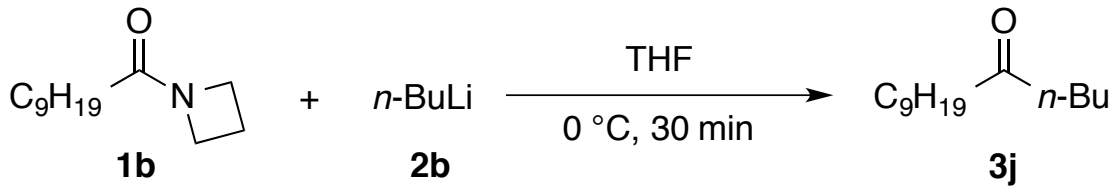

According to the general procedure, the reaction of 1-(azetidin-1-yl)decan-1-one $(0.50 \mathrm{mmol}$, $0.106 \mathrm{~g}, 1.00$ equiv), $n$-butyllithium (1.36 M solution in hexanes, $1.10 \mathrm{~mL}, 1.50 \mathrm{mmol}, 3.0$ equiv) in THF $(5.0 \mathrm{~mL})$ for $30 \mathrm{~min}$ at $0{ }^{\circ} \mathrm{C}$, afforded after work-up with $\mathrm{HCl}(1.0 \mathrm{~N}, 5 \mathrm{~mL})$ and chromatography the title compound in $89 \%$ yield $(94.6 \mathrm{mg})$. Colorless oil. GC: $\mathrm{rt}=13.00 \mathrm{~min}$. $\underline{\left.{ }^{1} \mathbf{H} \text { NMR (500 MHz, } \mathbf{C D C l}_{3}\right)} \delta 2.42-2.39(\mathrm{t}, J=7.5 \mathrm{~Hz}, 2 \mathrm{H}), 2.42-2.39(\mathrm{t}, J=7.4 \mathrm{~Hz}, 2 \mathrm{H})$, 1.60-1.54 (m, 4 H), 1.35-1.28 (m, 14 H), 0.94-0.91 (t, $J=7.3 \mathrm{~Hz}, 3 \mathrm{H}), 0.91-0.88$ (t, $J=7.1 \mathrm{~Hz}$, $3 \mathrm{H}) .{ }^{13} \mathbf{C}$ NMR (125 MHz, CDCl 3 ) $\delta$ 211.72, 42.84, 42.53, 31.87, 29.44, 29.43, 29.29, 29.28, 26.00, 23.91, 22.67, 22.39, 14.11, 13.87. $\underline{\mathbf{M S}}=212.2(\mathrm{EI})$. Spectroscopic data matched literature values. $^{14}$ 
1-Phenyldecan-1-one (Scheme 1, Entry 12)<smiles>O=C(Oc1ccccc1)c1ccccc1</smiles>

According to the general procedure, the reaction of 1-(azetidin-1-yl)decan-1-one $(0.50 \mathrm{mmol}$, $0.106 \mathrm{~g}, 1.00$ equiv), phenyllithium (1.90 $\mathrm{M}$ solution in $\mathrm{Bu}_{2} \mathrm{O}, 0.53 \mathrm{~mL}, 1.00 \mathrm{mmol}, 2.0$ equiv) in THF $(5.0 \mathrm{~mL})$ for $15 \mathrm{~min}$ at $-78{ }^{\circ} \mathrm{C}$, afforded after work-up with $\mathrm{HCl}(1.0 \mathrm{~N}, 5 \mathrm{~mL})$ and chromatography the title compound in $80 \%$ yield $(93.0 \mathrm{mg})$. Colorless oil. GC: $\mathrm{rt}=16.39 \mathrm{~min}$. $\underline{\left.{ }^{1} \mathbf{H} \text { NMR (500 MHz, } \mathbf{C D C l}_{3}\right)} \delta 8.99-7.98(\mathrm{~d}, J=7.3 \mathrm{~Hz}, 2 \mathrm{H}), 7.59-7.56(\mathrm{t}, J=7.4 \mathrm{~Hz}, 1 \mathrm{H})$, 7.50-7.47 (t, $J=7.8 \mathrm{~Hz}, 2 \mathrm{H}), 3.00-2.97$ (t, $J=7.4 \mathrm{~Hz}, 2 \mathrm{H}), 1.79-1.73$ (m, $2 \mathrm{H}), 1.39-1.29$ (m, $12 \mathrm{H}), 0.92-0.89(\mathrm{t}, J=6.7 \mathrm{~Hz}, 3 \mathrm{H}) .{ }^{13} \mathbf{C}$ NMR (125 MHz, CDCl 3 ) $\delta 200.60,137.12,132.84$, 128.54, 128.06, 38.65, 31.89, 29.50, 29.49, 29.40, 29.30, 24.41, 22.68, 14.12. $\underline{\mathbf{M S}}=232.2$ (EI). Spectroscopic data matched literature values. ${ }^{6}$

1-(Adamantan-1-yl)ethan-1-one (Scheme 1, Entry 13)

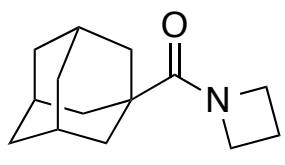

$1 c$

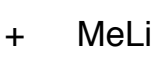

$2 \mathbf{a}$

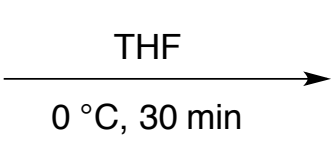

31

According to the general procedure, the reaction of (adamantan-1-yl)(azetidin-1-yl)methanone (0.50 mmol, $0.110 \mathrm{~g}, 1.00$ equiv), methyllithium (1.08 $\mathrm{M}$ solution in $\mathrm{Et}_{2} \mathrm{O}, 1.40 \mathrm{~mL}, 1.50 \mathrm{mmol}$, 3.0 equiv) in THF $(5.0 \mathrm{~mL})$ for $30 \mathrm{~min}$ at $0{ }^{\circ} \mathrm{C}$, afforded after work-up with $\mathrm{HCl}(1.0 \mathrm{~N}, 5 \mathrm{~mL})$ and chromatography the title compound in $93 \%$ yield $(82.9 \mathrm{mg})$. Colorless oil. $\underline{\mathbf{G C}} \mathbf{\mathrm { rt }}=11.57$ min. ${ }^{1} \mathbf{H}$ NMR (500 MHz, $\left.\mathbf{C D C l}_{3}\right) \delta 2.11(\mathrm{~s}, 3 \mathrm{H}) 2.06(\mathrm{~m}, 3 \mathrm{H}), 1.82-1.82(\mathrm{~m}, 6 \mathrm{H}), 1.78-1.69$ $(\mathrm{m}, 6 \mathrm{H}) . \underline{{ }^{13} \mathbf{C} \text { NMR }\left(125 \mathbf{~ M H z}, \mathbf{C D C l}_{3}\right)} \delta 214.14,46.50,38.27,36.56,27.95,24.31 . \underline{\mathbf{M S}}=178.1$ (EI). Spectroscopic data matched literature values. ${ }^{15}$

1-(Adamantan-1-yl)pentan-1-one (Scheme 1, Entry 14)

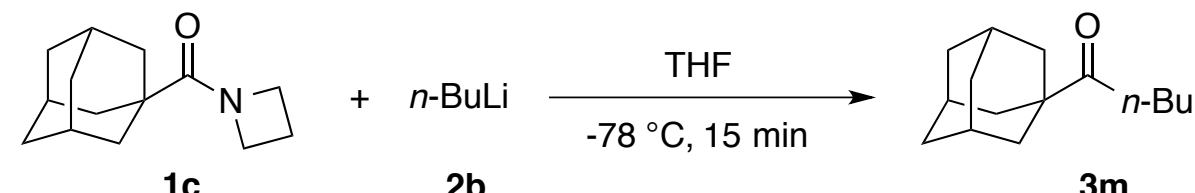


According to the general procedure, the reaction of (adamantan-1-yl)(azetidin-1-yl)methanone (0.50 mmol, $0.110 \mathrm{~g}, 1.00$ equiv), $n$-butyllithium (1.36 M solution in hexanes, $0.55 \mathrm{~mL}, 0.75$ mmol, 1.5 equiv) in THF $(5.0 \mathrm{~mL})$ for $15 \mathrm{~min}$ at $-78^{\circ} \mathrm{C}$, afforded after work-up with $\mathrm{HCl}(1.0 \mathrm{~N}$, $5 \mathrm{~mL})$ and chromatography the title compound in $84 \%$ yield $(92.6 \mathrm{mg})$. Colorless oil. $\underline{\text { GC: }} \mathrm{rt}=$ 15.01 min. ${ }^{1} \mathbf{H}$ NMR (500 MHz, $\left.\mathbf{C D C l}_{3}\right) \delta 2.45-2.42(\mathrm{t}, J=7.3 \mathrm{~Hz}, 2 \mathrm{H}), 2.04(\mathrm{~m}, 3 \mathrm{H}), 1.81$ 1.80 (m, $6 \mathrm{H}), 1.76-1.67$ (m, $6 \mathrm{H}), 1.55-1.49$ (m, $2 \mathrm{H}), 1.32-1.25$ (m, $2 \mathrm{H}), 0.92-0.89$ (t, $J=7.3$ $\mathrm{Hz}, 3 \mathrm{H}) .{ }^{13} \mathbf{C} \mathbf{N M R}\left(125 \mathbf{M H z}_{2} \mathbf{C D C l}_{3}\right) \delta 215.76,46.30,38.23,36.62,35.62,27.99,25.83$, $22.47,13.96 . \underline{\mathbf{M S}}=220.2(\mathrm{EI})$. Spectroscopic data matched literature values. ${ }^{16}$

(Adamantan-1-yl)(phenyl)methanone (Scheme 1, Entry 15)

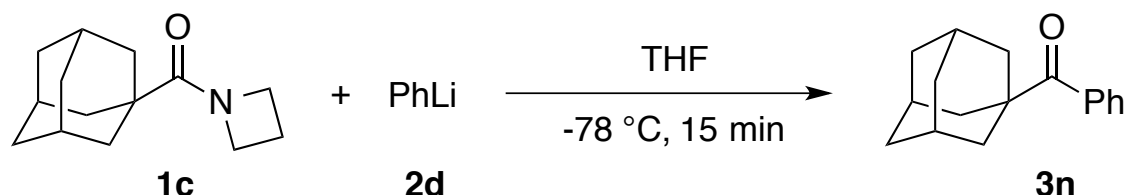

According to the general procedure, the reaction of (adamantan-1-yl)(azetidin-1-yl)methanone (0.50 mmol, $0.110 \mathrm{~g}, 1.00$ equiv), phenyllithium ( $1.90 \mathrm{M}$ solution in $\mathrm{Bu}_{2} \mathrm{O}, 0.79 \mathrm{~mL}, 1.50 \mathrm{mmol}$, 3.0 equiv) in THF $(5.0 \mathrm{~mL})$ for $15 \mathrm{~min}$ at $-78^{\circ} \mathrm{C}$, afforded after work-up with $\mathrm{HCl}(1.0 \mathrm{~N}, 5 \mathrm{~mL})$ and chromatography the title compound in 91\% yield $(109.4 \mathrm{mg})$. Light yellow oil. $\mathbf{\text { GC}}$ : $\mathrm{rt}=$ 18.14 min. ${ }^{1} \mathbf{H}$ NMR (500 MHz, $\left.\mathbf{C D C l}_{3}\right) \delta 7.57-7.55$ (d, $\left.J=7.0 \mathrm{~Hz}, 2 \mathrm{H}\right), 7.45-7.43(\mathrm{t}, J=7.1$ Hz, 1 H), 7.41-7.38 (t, $J=7.4$ Hz, 2 H), 2.09 (m, 3 H), 2.03-2.03 (m, 6 H), 1.79-1.73 (m, 6 H). ${ }^{13} \mathbf{C}$ NMR (125 MHz, CDCl $\left.{ }_{3}\right) \delta 210.06,139.62,130.15,127.95,127.13,46.90,39.12,36.56$, 28.16. $\underline{\text { MS }}=240.2(\mathrm{EI})$. Spectroscopic data matched literature values. ${ }^{17}$

Benzophenone (Scheme 2, Entry 1)

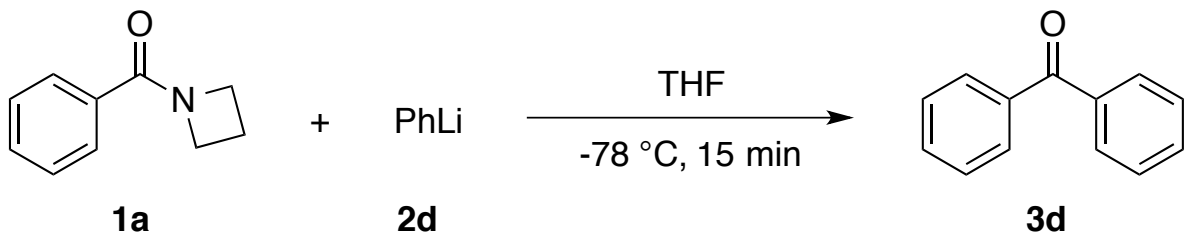

According to the general procedure, the reaction of azetidin-1-yl(phenyl)methanone $(0.50 \mathrm{mmol}$, $0.080 \mathrm{~g}, 1.00$ equiv), phenyllithium (1.90 $\mathrm{M}$ solution in $\mathrm{Bu}_{2} \mathrm{O}, 0.79 \mathrm{~mL}, 1.50 \mathrm{mmol}, 3.0$ equiv) in THF $(5.0 \mathrm{~mL})$ for $15 \mathrm{~min}$ at $-78{ }^{\circ} \mathrm{C}$, afforded after work-up with $\mathrm{HCl}(1.0 \mathrm{~N}, 5 \mathrm{~mL})$ and chromatography the title compound in $84 \%$ yield $(76.6 \mathrm{mg})$. Light yellow oil. GC: $\mathrm{rt}=13.75$ 
$\min . \underline{{ }^{1}} \underline{\mathbf{H} \text { NMR }\left(\mathbf{5 0 0} \mathbf{~ M H z}, \mathbf{C D C l}_{3}\right.} \underline{2} \delta$ 7.84-7.83 (d, $\left.J=7.1 \mathrm{~Hz}, 4 \mathrm{H}\right), 7.63-7.60(\mathrm{t}, J=7.5 \mathrm{~Hz}, 2$ H), 7.52-7.49 (t, $J=7.8 \mathrm{~Hz}, 4 \mathrm{H}) .{ }^{13} \mathbf{C}$ NMR (125 MHz, $\left.\mathbf{C D C l}_{3}\right) \delta 196.77,137.62,132.44$, $130.08,128.30 . \underline{\mathbf{M S}}=182.1$ (EI). Spectroscopic data matched literature values. ${ }^{10}$

(4-Methoxyphenyl)(phenyl)methanone (Scheme 2, Entry 2)

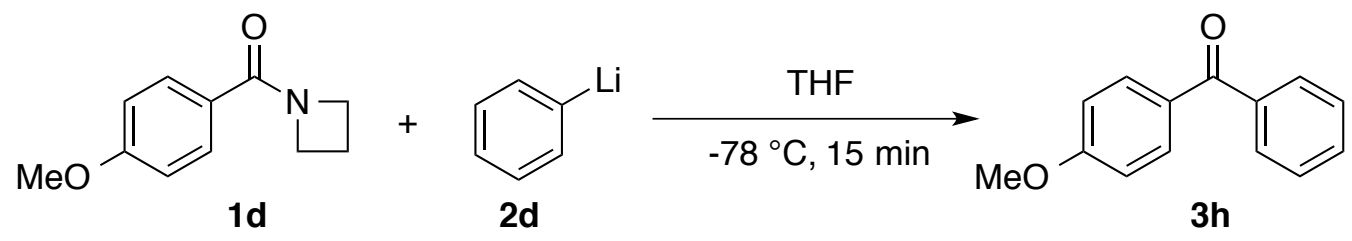

According to the general procedure, the reaction of azetidin-1-yl(4-methoxyphenyl)methanone (0.50 mmol, $0.096 \mathrm{~g}, 1.00$ equiv), phenyllithium ( $1.90 \mathrm{M}$ solution in $\mathrm{Bu}_{2} \mathrm{O}, 0.79 \mathrm{~mL}, 1.50 \mathrm{mmol}$, 3.0 equiv) in THF $(5.0 \mathrm{~mL})$ for $15 \mathrm{~min}$ at $-78{ }^{\circ} \mathrm{C}$, afforded after work-up with $\mathrm{HCl}(1.0 \mathrm{~N}, 5 \mathrm{~mL})$ and chromatography the title compound in $91 \%$ yield $(96.6 \mathrm{mg})$. Colorless oil. GC: $\mathrm{rt}=17.02$ $\min . \underline{{ }^{1} \mathbf{H} \text { NMR }\left(\mathbf{5 0 0} \mathbf{~ M H z}, \mathbf{C D C l}_{3}\right)} \delta 8.85-7.83(\mathrm{~d}, J=8.9 \mathrm{~Hz}, 2 \mathrm{H}), 7.78-7.76(\mathrm{~d}, J=7.1 \mathrm{~Hz}, 2$ H), 7.58-7.56 (t, $J=7.4 \mathrm{~Hz}, 1 \mathrm{H}), 7.49-7.46$ (t, $J=7.8 \mathrm{~Hz}, 2 \mathrm{H}), 6.98-6.96$ (d, $J=8.9 \mathrm{~Hz}, 2 \mathrm{H}$ ), 3.88 (s, 3 H). ${ }^{13} \mathbf{C}$ NMR (125 MHz, $\left.\mathbf{C D C l}_{3}\right) \delta 195.50,163.24,138.30,132.54,131.89,130.14$, $129.71,128.20,113.58,55.49 . \underline{\mathbf{M S}}=212.1$ (EI). Spectroscopic data matched literature values. ${ }^{8}$

Phenyl(p-tolyl)methanone (Scheme 2, Entry 3)<smiles>Cc1ccc(C(=O)N2CC[C@H]2C(=O)c2ccc([GeH3])cc2)cc1</smiles>

According to the general procedure, the reaction of azetidin-1-yl $(p$-tolyl $)$ methanone $(0.50 \mathrm{mmol}$, $0.088 \mathrm{~g}, 1.00$ equiv), phenyllithium (1.90 M solution in $\mathrm{Bu}_{2} \mathrm{O}, 0.79 \mathrm{~mL}, 1.50 \mathrm{mmol}, 3.0$ equiv) in THF $(5.0 \mathrm{~mL})$ for $15 \mathrm{~min}$ at $-78{ }^{\circ} \mathrm{C}$, afforded after work-up with $\mathrm{HCl}(1.0 \mathrm{~N}, 5 \mathrm{~mL})$ and chromatography the title compound in $93 \%$ yield $(91.3 \mathrm{mg})$. Yellow oil. $\underline{\text { GC: }} \mathrm{rt}=15.16 \mathrm{~min} . \underline{{ }^{1} \mathbf{H}}$ NMR (500 MHz, $\left.\mathbf{C D C l}_{3}\right) \delta 7.82-7.81(\mathrm{~d}, J=7.1 \mathrm{~Hz}, 2 \mathrm{H}), 7.76-7.75(\mathrm{~d}, J=8.1 \mathrm{~Hz}, 2 \mathrm{H}), 7.60-$ 7.57 (t, $J=7.4 \mathrm{~Hz}, 1 \mathrm{H}), 7.51-7.48$ (t, $J=7.8 \mathrm{~Hz}, 2 \mathrm{H}), 7.31-7.29$ (d, $J=8.0 \mathrm{~Hz}, 2 \mathrm{H}), 2.46$ (s, 3 H). ${ }^{13} \mathbf{C}$ NMR (125 MHz, $\left.\mathbf{C D C l}_{3}\right) \delta 196.44,143.24,137.98,134.91,132.18,130.31,129.93$, $129.00,128.23,21.67 . \underline{\mathbf{M S}}=196.1(\mathrm{EI})$. Spectroscopic data matched literature values. ${ }^{10}$ 
Phenyl(4-(trifluoromethyl)phenyl)methanone (Scheme 2, Entry 4)<smiles>O=C(c1ccccc1)c1ccc(C(F)(F)F)cc1</smiles>

According to the general procedure, the reaction of azetidin-1-yl(4-(trifluoromethyl)phenyl) methanone ( $0.50 \mathrm{mmol}, 0.115 \mathrm{~g}, 1.00$ equiv), phenyllithium ( $1.90 \mathrm{M}$ solution in $\mathrm{Bu}_{2} \mathrm{O}, 0.79 \mathrm{~mL}$, $1.50 \mathrm{mmol}, 3.0$ equiv) in THF $(5.0 \mathrm{~mL})$ for $15 \mathrm{~min}$ at $-78{ }^{\circ} \mathrm{C}$, afforded after work-up with $\mathrm{HCl}$ $(1.0 \mathrm{~N}, 5 \mathrm{~mL})$ and chromatography the title compound in 91\% yield $(113.9 \mathrm{mg})$. Yellow solid. GC: $\mathrm{rt}=13.16$ min. ${ }^{1} \mathbf{H}$ NMR (500 MHz, $\left.\mathbf{C D C l}_{3}\right) \delta 7.92-7.91(\mathrm{~d}, J=8.1 \mathrm{~Hz}, 2 \mathrm{H}), 7.84-7.82(\mathrm{~d}$, $J=7.3 \mathrm{~Hz}, 2 \mathrm{H}), 7.78-7.77(\mathrm{~d}, J=8.1 \mathrm{~Hz}, 2 \mathrm{H}), 7.66-7.63(\mathrm{t}, J=7.5 \mathrm{~Hz}, 1 \mathrm{H}), 7.54-7.51(\mathrm{t}, J=$ $7.9 \mathrm{~Hz}, 2 \mathrm{H}) .{ }^{13} \mathbf{C}$ NMR (125 MHz, $\left.\mathbf{C D C l}_{3}\right) \delta 195.49,140.73,136.73,133.70$ (q, $\left.J^{2}=32.5 \mathrm{~Hz}\right)$, 133.08, 130.14, 130.10, 128.53, 125.34 (q, $\left.J^{3}=3.7 \mathrm{~Hz}\right), 123.69$ (q, $\left.J^{l}=270.9 \mathrm{~Hz}\right) . \underline{{ }^{19} \mathbf{F} \text { NMR }}$ $\underline{\left(471 \mathbf{~ M H z}, \mathbf{C D C l}_{3}\right)}$ ) $\delta$-63.0. $\underline{\mathbf{M S}}=250.1(\mathrm{EI})$. Spectroscopic data matched literature values. ${ }^{10}$

(4-Fluorophenyl)(phenyl)methanone (Scheme 2, Entry 5)

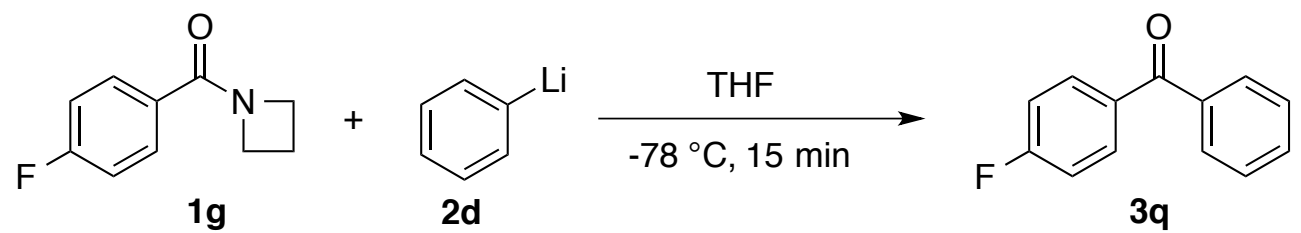

According to the general procedure, the reaction of azetidin-1-yl(4-fluorophenyl)methanone ( $0.50 \mathrm{mmol}, 0.090 \mathrm{~g}, 1.00$ equiv), phenyllithium $\left(1.90 \mathrm{M}\right.$ solution in $\mathrm{Bu}_{2} \mathrm{O}, 0.79 \mathrm{~mL}, 1.50 \mathrm{mmol}$, 3.0 equiv) in THF $(5.0 \mathrm{~mL})$ for $15 \mathrm{~min}$ at $-78^{\circ} \mathrm{C}$, afforded after work-up with $\mathrm{HCl}(1.0 \mathrm{~N}, 5 \mathrm{~mL})$ and chromatography the title compound in $81 \%$ yield $(81.1 \mathrm{mg})$. Yellow oil. GC: $\mathrm{rt}=13.50 \mathrm{~min}$. $\underline{{ }^{1} \mathbf{H} \text { NMR }\left(\mathbf{5 0 0 ~ M H z}, \mathbf{C D C l}_{3}\right)} \delta$ 7.88-7.85 (m, $\left.2 \mathrm{H}\right), 7.80-7.78(\mathrm{~d}, J=7.2 \mathrm{~Hz}, 2 \mathrm{H}), 7.62-7.60(\mathrm{t}, J$ $=7.5 \mathrm{~Hz}, 1 \mathrm{H}), 7.52-7.49(\mathrm{t}, J=7.8 \mathrm{~Hz}, 2 \mathrm{H}), 7.19-7.16(\mathrm{t}, J=8.7 \mathrm{~Hz}, 2 \mathrm{H}) . \underline{{ }^{13} \mathbf{C} \text { NMR }(\mathbf{1 2 5}}$ $\underline{\text { MHz }, \mathbf{C D C l}_{3}} \underline{2} \delta 195.24,165.38\left(\mathrm{~d}, J^{l}=252.5 \mathrm{~Hz}\right), 137.50,133.81\left(\mathrm{~d}, J^{4}=3.0 \mathrm{~Hz}\right), 132.67\left(\mathrm{~d}, J^{3}\right.$ $=9.1 \mathrm{~Hz}), 132.48,129.88,128.37,115.46\left(\mathrm{~d}, J^{2}=21.7 \mathrm{~Hz}\right) .{ }^{19} \mathbf{F}$ NMR $\left(471 \mathbf{~ M H z}, \mathbf{C D C l}_{3}\right) \delta-$ 106.0. $\underline{\text { MS }}=200.1$ (EI). Spectroscopic data matched literature values. ${ }^{10}$

(4-Chlorophenyl)(phenyl)methanone (Scheme 2, Entry 6) 
<smiles>CC(C)c1ccc(C(=O)c2ccc(Cl)cc2)cc1</smiles>

According to the general procedure, the reaction of azetidin-1-yl(4-chlorophenyl)methanone ( $0.50 \mathrm{mmol}, 0.098 \mathrm{~g}, 1.00$ equiv), phenyllithium (1.90 $\mathrm{M}$ solution in $\mathrm{Bu}_{2} \mathrm{O}, 0.79 \mathrm{~mL}, 1.50 \mathrm{mmol}$, 3.0 equiv) in THF $(5.0 \mathrm{~mL})$ for $15 \mathrm{~min}$ at $-78^{\circ} \mathrm{C}$, afforded after work-up with $\mathrm{HCl}(1.0 \mathrm{~N}, 5 \mathrm{~mL})$ and chromatography the title compound in $89 \%$ yield $(96.5 \mathrm{mg})$. Light yellow oil. $\mathbf{G C}$ : $\mathrm{rt}=$ $15.71 \mathrm{~min} .{ }^{1} \mathbf{H}$ NMR (500 MHz, $\left.\mathbf{C D C l}_{3}\right) \delta$ 7.81-7.77 (t, $\left.J=8.8 \mathrm{~Hz}, 2 \mathrm{H}\right), 7.80-7.76(\mathrm{t}, J=8.8$ $\mathrm{Hz}, 2 \mathrm{H}), 7.63-7.60$ (t, $J=7.5 \mathrm{~Hz}, 1 \mathrm{H}), 7.52-7.49$ (t, $J=7.8 \mathrm{~Hz}, 2 \mathrm{H}), 7.48-7.47$ (d, $J=8.5 \mathrm{~Hz}, 2$ H). ${ }^{13} \mathbf{C}$ NMR (125 MHz, $\left.\mathbf{C D C l}_{3}\right) \delta 195.44,138.88,137.24,135.87,132.65,131.47,129.93$, 128.64, 128.41. $\underline{\mathbf{M S}}=216.0(\mathrm{EI})$. Spectroscopic data matched literature values. ${ }^{10}$

(4-Bromophenyl)(phenyl)methanone (Scheme 2, Entry 7)<smiles>O=C(c1ccc(Br)cc1)c1ccc(Br)cc1</smiles>

According to the general procedure, the reaction of azetidin-1-yl(4-bromophenyl)methanone ( $0.50 \mathrm{mmol}, 0.120 \mathrm{~g}, 1.00$ equiv), phenyllithium (1.90 $\mathrm{M}$ solution in $\mathrm{Bu}_{2} \mathrm{O}, 0.79 \mathrm{~mL}, 1.50 \mathrm{mmol}$, 3.0 equiv) in THF $(5.0 \mathrm{~mL})$ for $15 \mathrm{~min}$ at $-78^{\circ} \mathrm{C}$, afforded after work-up with $\mathrm{HCl}(1.0 \mathrm{~N}, 5 \mathrm{~mL})$ and chromatography the title compound in 93\% yield $(121.5 \mathrm{mg})$. Yellow solid. $\mathbf{G C}$ : $\mathrm{rt}=16.81$ $\min . \underline{\left.{ }^{1} \mathbf{H} \text { NMR (500 MHz, } \mathbf{C D C l}_{3}\right)} \delta 7.80-7.78(\mathrm{~d}, J=7.1 \mathrm{~Hz}, 2 \mathrm{H}), 7.70-7.68(\mathrm{~d}, J=8.5 \mathrm{~Hz}, 2$ H), 7.65-7.60 (m, $3 \mathrm{H}), 7.52-7.49$ (t, $J=7.9 \mathrm{~Hz}, 2 \mathrm{H}) .{ }^{13} \mathbf{C}$ NMR (125 MHz, CDCl $\left.{ }_{3}\right) \delta 195.56$, 137.18, 136.32, 132.69, 131.62, 131.57, 129.94, 128.43, 127.51. $\underline{\mathbf{M S}}=260.0$ (EI). Spectroscopic data matched literature values. ${ }^{18}$

(2,4-Dimethoxyphenyl)(4-(trifluoromethyl)phenyl)methanone (Scheme 2, Entry 8)<smiles>CCN(C)C(=O)c1ccc(C(F)(F)F)cc1</smiles>

$1 f$<smiles>COc1ccc(Cl)c(OC)c1</smiles>

2j<smiles>COc1ccc(C(=O)c2ccc(C(F)(F)F)cc2)c(OC)c1</smiles>

3t 
According to the general procedure, the reaction of (2,4-dimethoxyphenyl)lithium (prepared from 1-bromo-2,4-dimethoxybenzene ( $1.25 \mathrm{mmol}, 0.271 \mathrm{~g}, 2.50$ equiv), $n$-butyllithium (1.36 M solution in hexanes, $0.74 \mathrm{~mL}, 1.00 \mathrm{mmol}, 2.0$ equiv) in THF $(2.0 \mathrm{~mL})$ for $\left.1 \mathrm{~h} \mathrm{at}-78{ }^{\circ} \mathrm{C}\right)$, azetidin-1-yl(4-(trifluoromethyl)phenyl)methanone (0.50 mmol, $0.115 \mathrm{~g}, 1.00$ equiv) and THF (3.0 mL) for $1 \mathrm{~h}$ at $-78{ }^{\circ} \mathrm{C}$, afforded after work-up with $\mathrm{HCl}(1.0 \mathrm{~N}, 5 \mathrm{~mL})$ and chromatography the title compound in 90\% yield $(139.7 \mathrm{mg})$. White solid. GC: $\mathrm{rt}=17.64 \mathrm{~min} . \underline{{ }^{1} \mathbf{H} \text { NMR (500 }}$ $\underline{\left.\mathrm{MHz}, \mathbf{C D C l}_{3}\right)} \delta$ 7.84-7.82 (d, $\left.J=8.2 \mathrm{~Hz}, 2 \mathrm{H}\right), 7.67-7.65(\mathrm{~d}, J=8.2 \mathrm{~Hz}, 2 \mathrm{H}), 7.48-7.46(\mathrm{~d}, J=$ 8.5 Hz, $1 \mathrm{H}), 6.58-6.56(\mathrm{~m}, 1 \mathrm{H}), 6.51-6.50(\mathrm{~d}, J=2.1 \mathrm{~Hz}, 1 \mathrm{H}), 3.86(\mathrm{~s}, 3 \mathrm{H}), 3.65(\mathrm{~s}, 3 \mathrm{H}) .{ }^{13} \mathbf{C}$ $\underline{\left.\text { NMR (125 MHz, } \mathbf{C D C l}_{3}\right)} \delta 194.28,162.02\left(\mathrm{~d}, J^{l}=529.1 \mathrm{~Hz}\right), 142.24,133.25\left(\mathrm{q}, J^{2}=32.2 \mathrm{~Hz}\right)$, $132.67,129.56,127.08,124.96\left(\mathrm{q}, J^{3}=3.6 \mathrm{~Hz}\right), 122.75,120.55,105.08,98.66,55.50,55.39 .{ }^{19} \mathbf{F}$

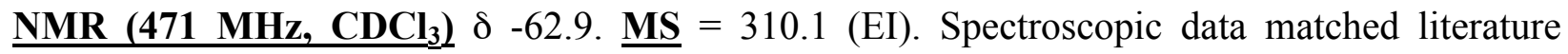
values. $^{19}$

(4-Chlorophenyl)(pyridin-2-yl)methanone (Scheme 2, Entry 9)<smiles>O=C(c1ccc(Cl)cc1)c1ccccn1</smiles>

According to the general procedure, the reaction of 2-pyridinyllithium (prepared from 2bromopyridine (1.25 mmol, $0.198 \mathrm{~g}, 2.50$ equiv), $n$-butyllithium (1.36 M solution in hexanes, $0.74 \mathrm{~mL}, 1.00 \mathrm{mmol}, 2.0$ equiv) in THF $(2.0 \mathrm{~mL})$ for $1 \mathrm{~h}$ at $\left.-78{ }^{\circ} \mathrm{C}\right)$, azetidin-1-yl(4chlorophenyl)methanone ( $0.50 \mathrm{mmol}, 0.098 \mathrm{~g}, 1.00$ equiv) and THF $(3.0 \mathrm{~mL})$ for $1 \mathrm{~h}$ at $-78{ }^{\circ} \mathrm{C}$, afforded after work-up with $\mathrm{HCl}(1.0 \mathrm{~N}, 5 \mathrm{~mL})$ and chromatography the title compound in $85 \%$

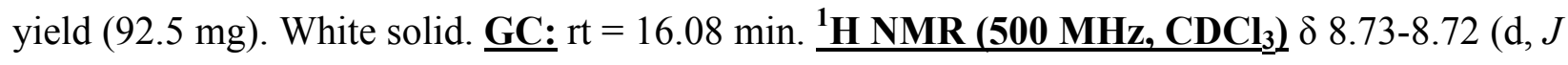
$=4.2 \mathrm{~Hz}, 1 \mathrm{H}), 8.09-8.07(\mathrm{~m}, 3 \mathrm{H}), 7.94-7.90(\mathrm{~m}, 1 \mathrm{H}), 7.52-7.50(\mathrm{~m}, 1 \mathrm{H}), 7.48-7.46(\mathrm{~d}, J=8.6$ $\mathrm{Hz}, 2 \mathrm{H}) .{ }^{13} \mathbf{C} \mathbf{N M R}\left(\mathbf{1 2 5} \mathbf{~ M H z}, \mathbf{C D C l}_{3} \mathbf{2} \delta 192.39,154.67,148.51,139.38,137.19,134.58\right.$, $132.50,128.46,126.42,124.68 . \underline{\mathbf{M S}}=217.0(\mathrm{EI})$. Spectroscopic data matched literature values. ${ }^{20}$

(4-Fluorophenyl)(1-methyl-1H-indol-5-yl)methanone (Scheme 2, Entry 10) 


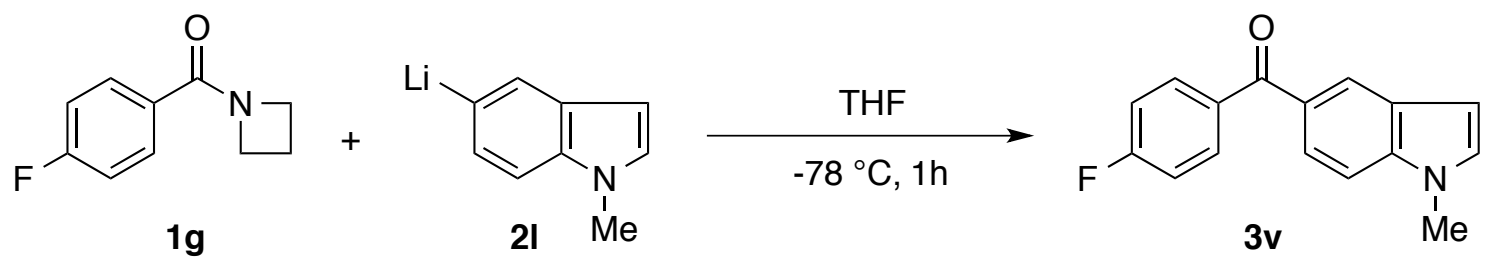

According to the general procedure, the reaction of (1-methyl-1H-indol-5-yl)lithium (prepared from 5-bromo-1-methyl-1 $H$-indole (1.25 mmol, $0.263 \mathrm{~g}, 2.50$ equiv), $n$-butyllithium (1.36 M solution in hexanes, $0.74 \mathrm{~mL}, 1.00 \mathrm{mmol}, 2.0$ equiv) in THF $(2.0 \mathrm{~mL})$ for $\left.1 \mathrm{~h} \mathrm{at}-78{ }^{\circ} \mathrm{C}\right)$, azetidin-1-yl(4-fluorophenyl)methanone $(0.50 \mathrm{mmol}, 0.090 \mathrm{~g}, 1.00$ equiv) and THF (3.0 mL) for $1 \mathrm{~h}$ at $-78{ }^{\circ} \mathrm{C}$, afforded after work-up with $\mathrm{HCl}(1.0 \mathrm{~N}, 5 \mathrm{~mL})$ and chromatography the title compound in $63 \%$ yield $(79.8 \mathrm{mg})$. New compound. White solid. Mp: $112-113{ }^{\circ} \mathrm{C}$. GC: $\mathrm{rt}=$ 20.82 min. ${ }^{1}$ H NMR (500 MHz, $\left.\mathbf{C D C l}_{3}\right) \delta 8.11$ (s, $\left.1 \mathrm{H}\right), 7.88-7.85$ (m, $\left.2 \mathrm{H}\right), 7.82-7.80(\mathrm{~d}, J=$ 8.6 Hz, $1 \mathrm{H}), 7.41-7.40(\mathrm{~d}, J=8.7 \mathrm{~Hz}, 1 \mathrm{H}), 7.19-7.15(\mathrm{~m}, 3 \mathrm{H}), 6.61-6.61(\mathrm{~d}, J=3.0 \mathrm{~Hz}, 1 \mathrm{H})$, 3.85 (s, $3 \mathrm{H}) .{ }^{13} \mathbf{C}$ NMR (125 MHz, $\left.\mathbf{C D C l}_{3}\right) \delta 195.79,164.89\left(\mathrm{~d}, J^{l}=250.9 \mathrm{~Hz}\right), 138.99,135.26$, $132.41\left(\mathrm{~d}, J^{3}=8.9 \mathrm{~Hz}\right), 130.58,129.05,127.70,125.19,123.68,115.17\left(\mathrm{~d}, J^{2}=21.6 \mathrm{~Hz}\right)$, 109.20, 102.94, 33.07. ${ }^{19}$ F NMR (471 MHz, $\mathbf{C D C l}_{3}$ ) $\delta$-107.6. $\underline{\text { MS }}=253.1$ (EI). $\underline{\text { HRMS }}$ calcd for $\mathrm{C}_{16} \mathrm{H}_{11} \mathrm{ONF}\left(\mathrm{M}^{+}-\mathrm{H}\right) 252.0819$ found 252.0825 .

(E)-3-(4-Methoxyphenyl)-1-phenylprop-2-en-1-one (Scheme 2, Entry 11)<smiles>COc1ccc(/C=C/C(=O)[C@@H]2CCN2C(=O)/C=C/c2ccc(OC)cc2)cc1</smiles>

According to the general procedure, the reaction of (E)-1-(azetidin-1-yl)-3-(4methoxyphenyl)prop-2-en-1-one (0.50 mmol, $0.109 \mathrm{~g}, 1.00$ equiv), phenyllithium (1.90 M solution in $\mathrm{Bu}_{2} \mathrm{O}, 0.79 \mathrm{~mL}, 1.50 \mathrm{mmol}, 3.0$ equiv) in $\mathrm{THF}(5.0 \mathrm{~mL})$ for $15 \mathrm{~min}$ at $-78{ }^{\circ} \mathrm{C}$, afforded after work-up with $\mathrm{HCl}(1.0 \mathrm{~N}, 5 \mathrm{~mL})$ and chromatography the title compound in $87 \%$

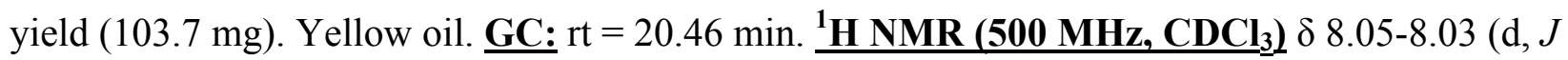
$=7.2 \mathrm{~Hz}, 2 \mathrm{H}), 7.83-7.80(\mathrm{~d}, J=15.7 \mathrm{~Hz}, 1 \mathrm{H}), 7.63-7.61(\mathrm{~d}, J=8.8 \mathrm{~Hz}, 2 \mathrm{H}), 7.59-7.58(\mathrm{~d}, J=$ $7.4 \mathrm{~Hz}, 1 \mathrm{H}), 7.53-7.50$ (t, $J=7.8 \mathrm{~Hz}, 2 \mathrm{H}), 7.46-7.43$ (d, $J=15.6 \mathrm{~Hz}, 1 \mathrm{H}), 6.96-6.95$ (d, $J=8.8$ $\mathrm{Hz}, 2 \mathrm{H}), 3.86$ (s, $3 \mathrm{H}) .{ }^{13} \mathbf{C}$ NMR (125 MHz, $\left.\mathbf{C D C l}_{3}\right) \delta 190.59,161.71,144.75,138.51,132.60$, 
130.27, 128.59, 128.44, 127.62, 119.77, 114.45, 55.42. $\underline{\mathbf{M S}}=238.1$ (EI). Spectroscopic data matched literature values. ${ }^{21}$ 


\section{Selectivity Studies: Amides}

General Procedure. An oven-dried vial equipped with a stir bar was charged with two amide substrates (each $0.5 \mathrm{mmol}, 2.0$ equiv), placed under a positive pressure of nitrogen, and subjected to three evacuation/backfilling cycles. Tetrahydrofuran $(5.0 \mathrm{~mL}, 0.10 \mathrm{M})$ was added at room temperature, the reaction was cooled down to $-78^{\circ} \mathrm{C}$, an organometallic reagent $(0.25 \mathrm{mmol}, 1.0$ equiv) was added dropwise with vigorous stirring at $-78^{\circ} \mathrm{C}$, and the reaction mixture was stirred at $-78{ }^{\circ} \mathrm{C}$ for $15 \mathrm{~min}$. After the indicated time, the reaction mixture was quenched by slow addition of $\mathrm{HCl}(1.0 \mathrm{~N}, 5 \mathrm{~mL})$, extracted with $\mathrm{Et}_{2} \mathrm{O}(2 \times 30 \mathrm{~mL})$, organic layers were combined, dried, filtered and concentrated. The sample was analyzed by ${ }^{1} \mathrm{H} \mathrm{NMR}\left(\mathrm{CDCl}_{3}, 500 \mathrm{MHz}\right)$ and GC-MS to obtain conversion and yield using internal standard and comparison with authentic samples.

Table SI-1. Selectivity Study in the Synthesis of Ketones by the Addition of Organometallics to $N$-Acylazetidines. ${ }^{a}$

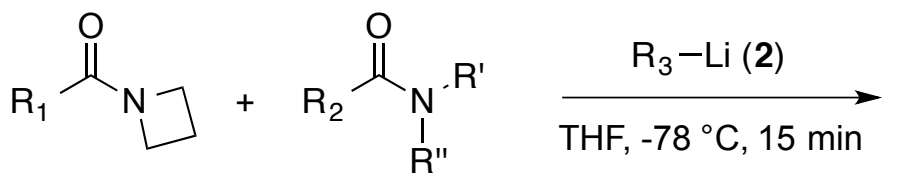

$1-1$
$1-$ II<smiles></smiles>

3-I
3-II

\begin{tabular}{|c|c|c|c|c|c|}
\hline Entry & $\begin{array}{c}1-I \\
\left(R_{1}\right)\end{array}$ & $\begin{array}{c}1-\text { II } \\
\left.\left(\mathrm{R}_{2} / \mathrm{NR}^{\prime} \mathrm{R}^{\prime},\right)^{\prime}\right)\end{array}$ & $\begin{array}{c}\mathbf{2} \\
\left(\mathrm{R}_{3} \mathrm{Li}\right)\end{array}$ & $\begin{array}{l}\text { Amide } \\
\text { (equiv) }\end{array}$ & $\begin{array}{l}\text { 3-I:3-II } \\
\left(\mathrm{R}_{1}: \mathrm{R}_{2}\right)^{b}\end{array}$ \\
\hline 1 & $4-\mathrm{Me}-\mathrm{C}_{6} \mathrm{H}_{4}$ & $\mathrm{C}_{6} \mathrm{H}_{5} / \mathrm{N}(\mathrm{Me})(\mathrm{OMe})$ & $\mathrm{PhLi}$ & 2.0 & $1.94: 1.0$ \\
\hline 2 & $4-\mathrm{Me}-\mathrm{C}_{6} \mathrm{H}_{4}$ & $\mathrm{C}_{6} \mathrm{H}_{5} / \mathrm{N}(\mathrm{Me})(\mathrm{OMe})$ & $\mathrm{MeLi}$ & 2.0 & $2.00: 1.0$ \\
\hline 3 & $4-\mathrm{Me}-\mathrm{C}_{6} \mathrm{H}_{4}$ & $\mathrm{C}_{6} \mathrm{H}_{5} / \mathrm{NMe}_{2}$ & PhLi & 2.0 & $3.00: 1.0$ \\
\hline 4 & $4-\mathrm{Me}-\mathrm{C}_{6} \mathrm{H}_{4}$ & $\mathrm{C}_{6} \mathrm{H}_{5} / \mathrm{N}\left(\mathrm{CH}_{2} \mathrm{CH}\right) \mathrm{Me}^{c}$ & $\mathrm{PhLi}$ & 2.0 & $1.72: 1.0$ \\
\hline
\end{tabular}

${ }^{a}$ Conditions: amide (2.0 equiv), $\mathrm{R}_{3} \mathrm{Li}\left(1.0\right.$ equiv), THF $(0.10 \mathrm{M}),-78{ }^{\circ} \mathrm{C}$. All reactions carried out using standard Schlenk techniques under nitrogen. ${ }^{b}$ Determined by ${ }^{1} \mathrm{H}$ NMR and/or GC-MS. ${ }^{c}$ (2-methylaziridin-1-yl)(phenyl)methanone. 


\section{Selectivity Studies: Sterics and Electronics}

General Procedure. An oven-dried vial equipped with a stir bar was charged with two amide substrates (each $0.5 \mathrm{mmol}, 2.0$ equiv), placed under a positive pressure of nitrogen, and subjected to three evacuation/backfilling cycles. Tetrahydrofuran $(5.0 \mathrm{~mL}, 0.10 \mathrm{M})$ was added at room temperature, the reaction was cooled down to $-78^{\circ} \mathrm{C}$, an organometallic reagent $(0.25 \mathrm{mmol}, 1.0$ equiv) was added dropwise with vigorous stirring at $-78{ }^{\circ} \mathrm{C}$, and the reaction mixture was stirred at $-78{ }^{\circ} \mathrm{C}$ for $15 \mathrm{~min}$. After the indicated time, the reaction mixture was quenched by slow addition of $\mathrm{HCl}(1.0 \mathrm{~N}, 5 \mathrm{~mL})$, extracted with $\mathrm{Et}_{2} \mathrm{O}(2 \times 30 \mathrm{~mL})$, organic layers were combined, dried, filtered and concentrated. The sample was analyzed by ${ }^{1} \mathrm{H} \mathrm{NMR}\left(\mathrm{CDCl}_{3}, 500 \mathrm{MHz}\right)$ and GC-MS to obtain conversion and yield using internal standard and comparison with authentic samples.

Table SI-2. Selectivity Study in the Synthesis of Ketones by the Addition of Organometallics to $N$-Acylazetidines. ${ }^{a}$<smiles>[R]C(=O)N1CCC1</smiles>

$1-1$
1-II

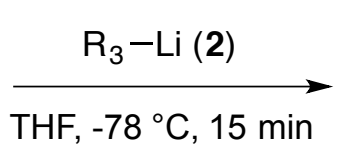

THF, $-78^{\circ} \mathrm{C}, 15 \mathrm{~min}$<smiles></smiles>

3-I
3-II

\begin{tabular}{cccccc}
\hline Entry & $\mathbf{1 - I}$ & $\mathbf{1 - I I}$ & $\mathbf{2}$ & Amide & 3-I:3-II \\
& $\left(\mathrm{R}_{1}\right)$ & $\left(\mathrm{R}_{2}\right)$ & $\left(\mathrm{R}_{3} \mathrm{Li}\right)$ & $\begin{array}{c}\text { (equiv) } \\
\left(\mathrm{R}_{1}: \mathrm{R}_{2}\right)^{b}\end{array}$ \\
\hline 1 & $4-\mathrm{CF}_{3}-\mathrm{C}_{6} \mathrm{H}_{4-}$ & $4-\mathrm{MeO}-\mathrm{C}_{6} \mathrm{H}_{4^{-}}$ & $\mathrm{PhLi}$ & 2.0 & $3.30: 1.0$ \\
2 & $\mathrm{C}_{6} \mathrm{H}_{5-}$ & $\mathrm{C}_{9} \mathrm{H}_{19^{-}}$ & $\mathrm{PhLi}$ & 2.0 & $2.82: 1.0$ \\
3 & $\mathrm{C}_{9} \mathrm{H}_{19^{-}}$ & $\mathrm{Ad}-$ & $\mathrm{PhLi}$ & 2.0 & $>20: 1.0$ \\
\hline
\end{tabular}

${ }^{a}$ Conditions: amide (2.0 equiv), $\mathrm{R}_{3} \mathrm{Li}\left(1.0\right.$ equiv), $\mathrm{THF}(0.10 \mathrm{M}),-78{ }^{\circ} \mathrm{C}$. All reactions carried out using standard Schlenk techniques under nitrogen. ${ }^{b}$ Determined by ${ }^{1} \mathrm{H}$ NMR and/or GC-MS. 


\section{Effect of Lewis Acid Additives}

General Procedure. An oven-dried vial equipped with a stir bar was charged with an amide substrate ( $0.5 \mathrm{mmol}, 1.0$ equiv), placed under a positive pressure of nitrogen, and subjected to three evacuation/backfilling cycles. Tetrahydrofuran $(5.0 \mathrm{~mL}, 0.10 \mathrm{M})$ and Lewis acid additive (3.0 equiv) were added at room temperature, the reaction was cooled down to $-78{ }^{\circ} \mathrm{C}$, an organometallic reagent (1.0 mmol, 2.0 equiv) was added dropwise with vigorous stirring at -78 ${ }^{\circ} \mathrm{C}$, and the reaction mixture was stirred at $-78{ }^{\circ} \mathrm{C}$ for $1 \mathrm{~h}$. After the indicated time, the reaction mixture was quenched by slow addition of $\mathrm{HCl}(1.0 \mathrm{~N}, 5 \mathrm{~mL})$, extracted with $\mathrm{Et}_{2} \mathrm{O}(2 \times 30 \mathrm{~mL})$, organic layers were combined, dried, filtered and concentrated. The sample was analyzed by ${ }^{1} \mathrm{H}$ NMR $\left(\mathrm{CDCl}_{3}, 500 \mathrm{MHz}\right)$ and GC-MS to obtain conversion and yield using internal standard and comparison with authentic samples. Note: In the absence of additives, $>95 \%$ conversion ( $>90 \%$ yield) is observed after $15 \mathrm{~min}$ reaction time $-78^{\circ} \mathrm{C}$. The observed results are consistent with a switchable N-/O-coordination of $\mathrm{N}$-acylazetidinyl amides under these reaction conditions $\left(\chi_{\mathrm{N}}=\right.$ $32.5^{\circ} ; \tau=3.3^{\circ}$, azetidin-1-yl( $p$-tolyl)methanone).$^{23}$ It has been shown that $\mathrm{N}$-coordination precedes transmetallation in the nucleophilic addition of organometallics to carbonyl electrophiles. $^{24}$

Table SI-3. Effect of Lewis Acid Additives on the Stability of Tetrahedral Intermediate in the Nucleophilic Addition to $N$-Acylazetidines. ${ }^{a}$

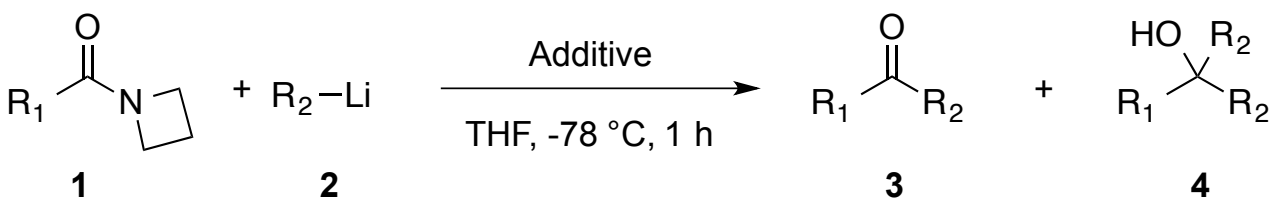

\begin{tabular}{ccccccc}
\hline Entry & $\mathbf{1 - I}$ & $\mathbf{2}$ & Additive & Yield & Conv. & Selectivity \\
& $\left(\mathrm{R}_{1}\right)$ & $\left(\mathrm{R}_{2} \mathrm{Li}\right)$ & & $(\%)^{b}$ & $(\%)^{b}$ & $(\%)^{b}$ \\
\hline 1 & $\mathrm{C}_{6} \mathrm{H}_{5^{-}}$ & $\mathrm{PhLi}$ & $\mathrm{LiCl}$ & 48 & 67 & $>98: 2$ \\
2 & $\mathrm{C}_{6} \mathrm{H}_{5^{-}}$ & $\mathrm{PhLi}$ & $\mathrm{ZnCl}_{2}$ & 42 & 58 & $>98: 2$ \\
3 & $\mathrm{C}_{6} \mathrm{H}_{5-}$ & $\mathrm{PhLi}$ & $\mathrm{BF}_{3} \bullet \mathrm{Et}_{2} \mathrm{O}$ & 45 & 71 & $>98: 2$ \\
\hline
\end{tabular}

${ }^{a}$ Conditions: amide (2.0 equiv), $\mathrm{R}_{3} \mathrm{Li}\left(1.0\right.$ equiv), THF $(0.10 \mathrm{M}),-78{ }^{\circ} \mathrm{C}$. All reactions carried out using standard Schlenk techniques under nitrogen. ${ }^{b}$ Determined by ${ }^{1} \mathrm{H}$ NMR and/or GC-MS. 


\section{References}

1. Otani, Y.; Nagae, O.; Naruse, Y.; Inagaki, S.; Ohno, M.; Yamaguchi, K.; Yamamoto, G.; Uchiyama, M.; Ohwada, T. J. Am. Chem. Soc. 2003, 125, 15191.

2. Kiuchi, F.; Nishizawa, S.; Kawanishi, H.; Kinoshita, S.; Ohsima, H.; Uchitani, A.; Sekino, N.; Ishida, M.; Kondo, K.; Tsuda, Y. Chem. Pharm. Bull. 1992, 12, 3234.

3. Au, V.; Harichian, B.; Cloudsdale, I. S.; Bajor, J. S.; Dickson, J. J. WO 2014139965 A1.

4. Bailey, J. M.; Bruton, G.; Huxley, A.; Milner, P. H.; Orlek, B. S. WO 2005014571 A1.

5. McKerrecher, D.; Pike, K. G.; Waring, M. J. WO 2007017649 A1.

6. Meng, G.; Szostak, M. Org. Lett. 2015, 17, 4364.

7. Cabrero-Antonino, J. R.; Alberico, E.; Drexler, H. J.; Baumann, W.; Junge, K.; Junge, H.; Beller, M. ACS. Catal. 2016, 6, 47.

8. Sheng, W. B.; Jiang, Q.; Luo, W. P.; Guo, C. C. J. Org. Chem. 2013, 78, 5691.

9. Shibuya, M.; Osada, Y.; Sasano, Y.; Tomizawa, M.; Iwabuchi, Y. J. Am. Chem. Soc. 2011, 133, 6497.

10. Zhou, Q.; Wei, S.; Han, W. J. Org. Chem. 2014, 79, 1454.

11. Whittaker, R. E.; Dong, G. Org. Lett. 2015, 17, 5504.

12. Xin, B.; Zhang, Y.; Cheng, K. J. Org. Chem. 2006, 71, 5725.

13. Kalutharage, N.; Yi, C. S. J. Am. Chem. Soc. 2015, 137, 11105.

14. Takahashi, M.; Oshima, K.; Matsubara, S. Tetrahedron. Lett. 2003, 44, 9201.

15. Wright, J. A.; Gaunt, M. J.; Spencer, J. B. Chem. Eur. J. 2006, 12, 949.

16. Pimenov, A. A.; Zemtsova, M. N.; Makarova, N. V.; Moiseev, I. K. Chem. Heterocycl. Comp. 2000, 36, 15.

17. Xiao, B.; Gong, T. J.; Xu, J.; Liu, Z. J.; Liu, L. J. Am. Chem. Soc. 2011, 133, 1466.

18. Ishiyama, T.; Kizaki, H.; Hayashi, T.; Suzuki, A.; Miyaura, N. J. Org. Chem. 1998, 63, 4726.

19. Gray, D. N.; Knight, R. D. US 3387035.

20. Yang, H.; Huo, N.; Yang, P.; Pei, H.; Lv, H.; Zhang, X. Org. Lett. 2015, 17, 4144.

21. Zhu, Y.; Li, C.; Zhang, J.; She, M.; Sun, W.; Wan, K.; Wang, Y.; Yin, B.; Liu, P.; Li, J. Org. Lett. 2015, 17, 3872.

22. Krasovskiy, A.; Knochel, P. Synthesis 2006, 890. 
23. Bennet, A. J.; Somayaji, V.; Brown, R. S.; Santarsiero, B. D. J. Am. Chem. Soc. 1991, 113,7563 .

24. Aubrecht, K. B.; Winemiller, M. D.; Collum, D. B. J. Am. Chem. Soc. 2000, 122, 11084. 


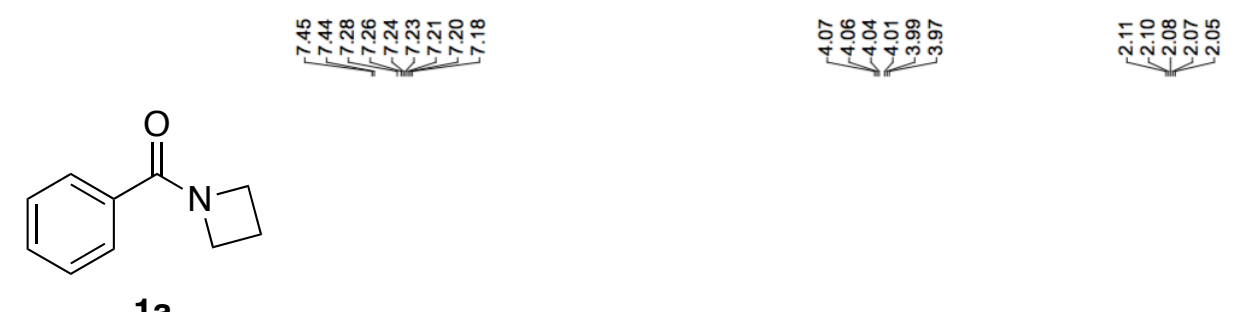

$1 a$
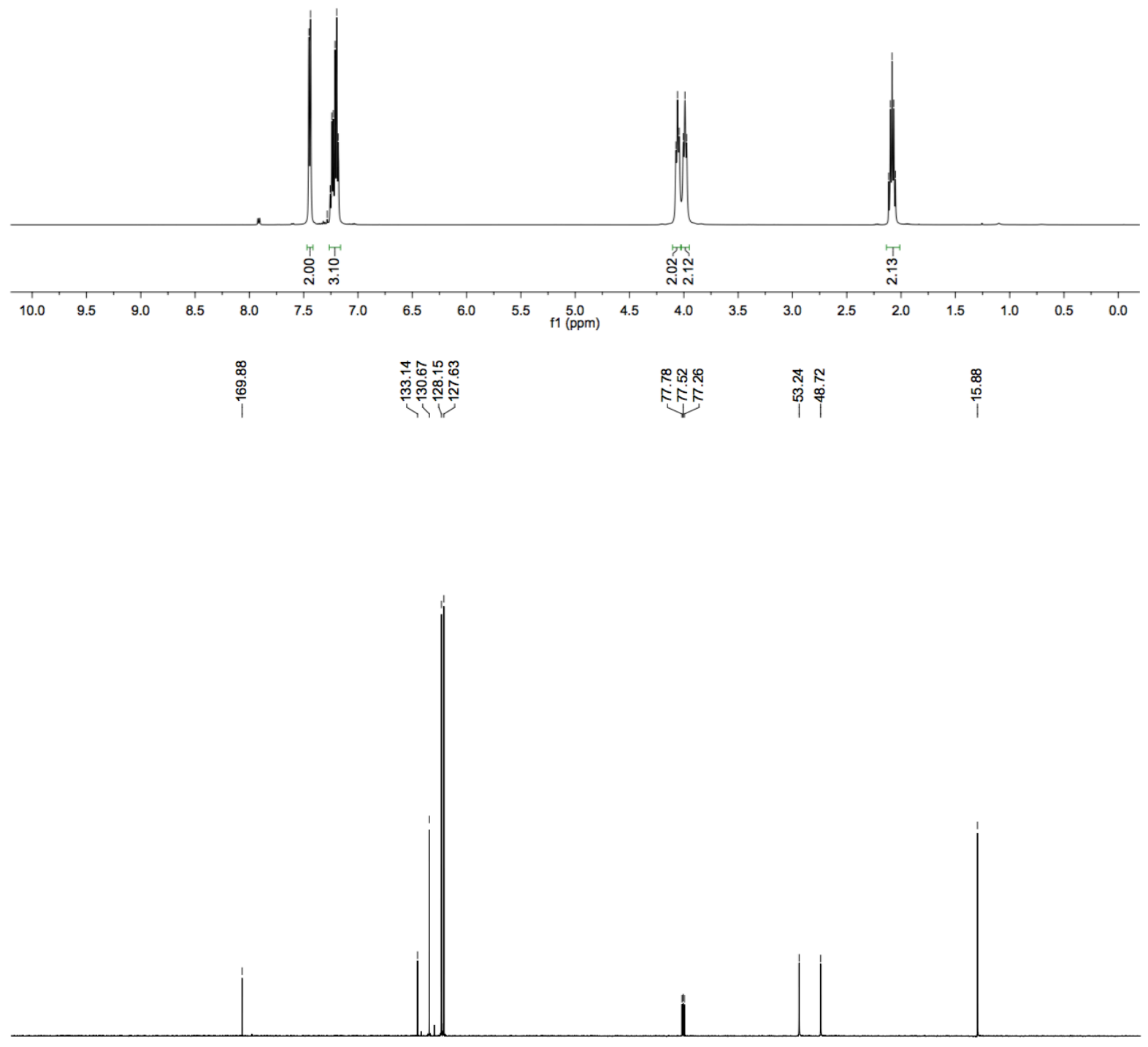

$\begin{array}{lllllllllllllllllllllllllll}210 & 200 & 190 & 180 & 170 & 160 & 150 & 140 & 130 & 120 & 110 & 100 & 90 & 80 & 70 & 60 & 50 & 40 & 30 & 20 & 10 & 0 & -10\end{array}$ 

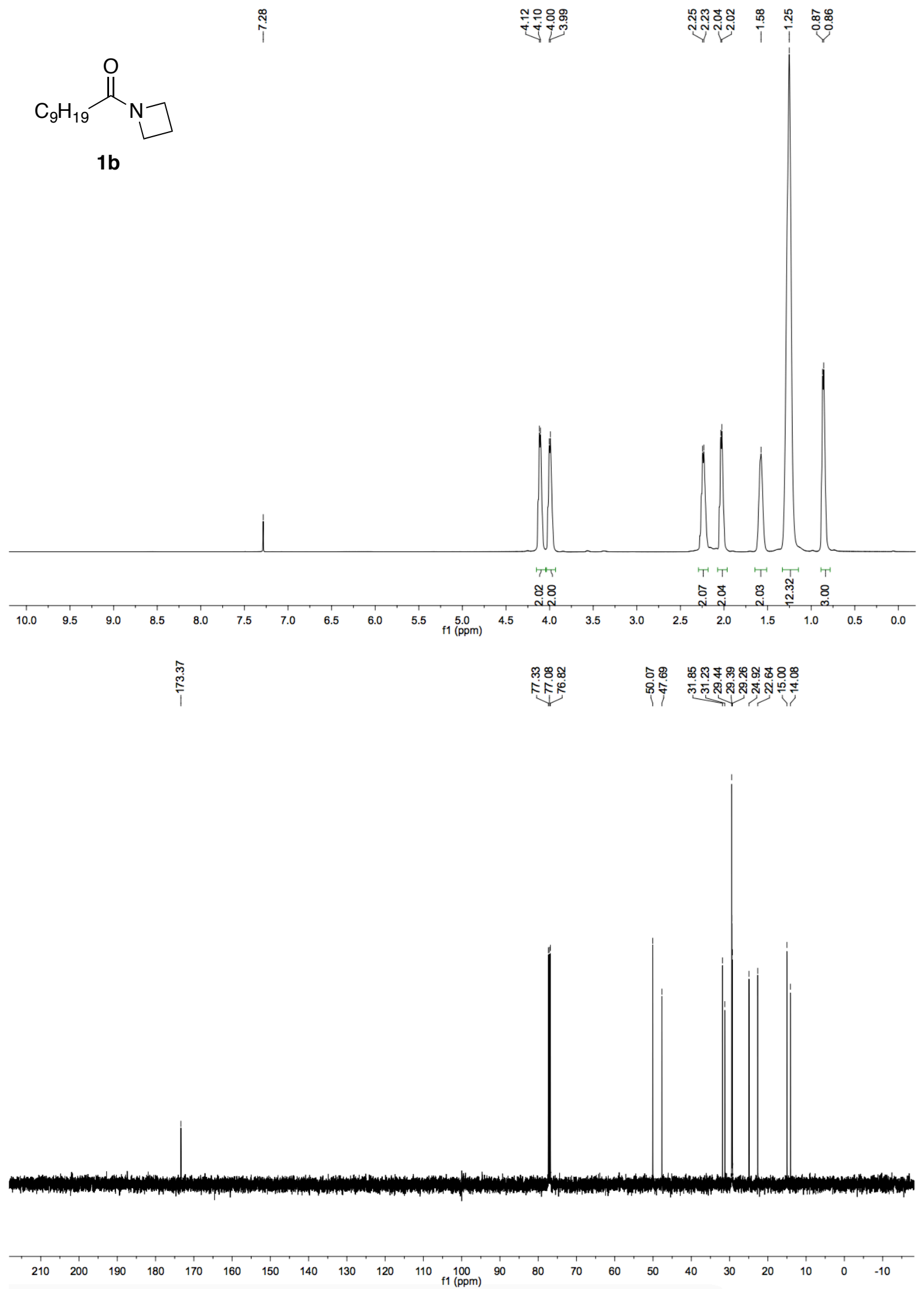

SI-27 

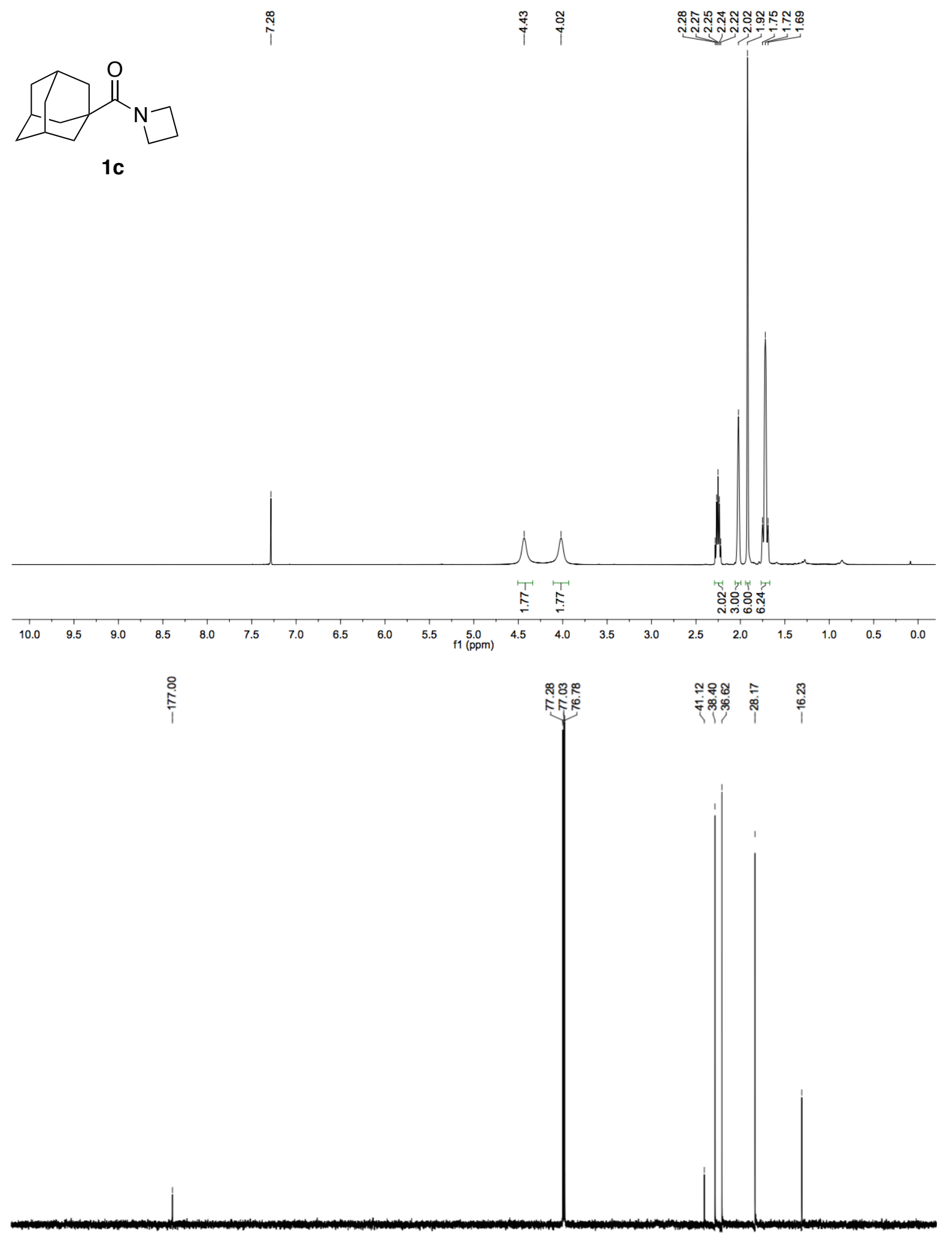

$\begin{array}{llllllllllllllllllllllllllllll}210 & 200 & 190 & 180 & 170 & 160 & 150 & 140 & 130 & 120 & 110 & 100 & 90 & 80 & 70 & 60 & 50 & 40 & 30 & 20 & 10 & 0 & -10\end{array}$ SI-28 

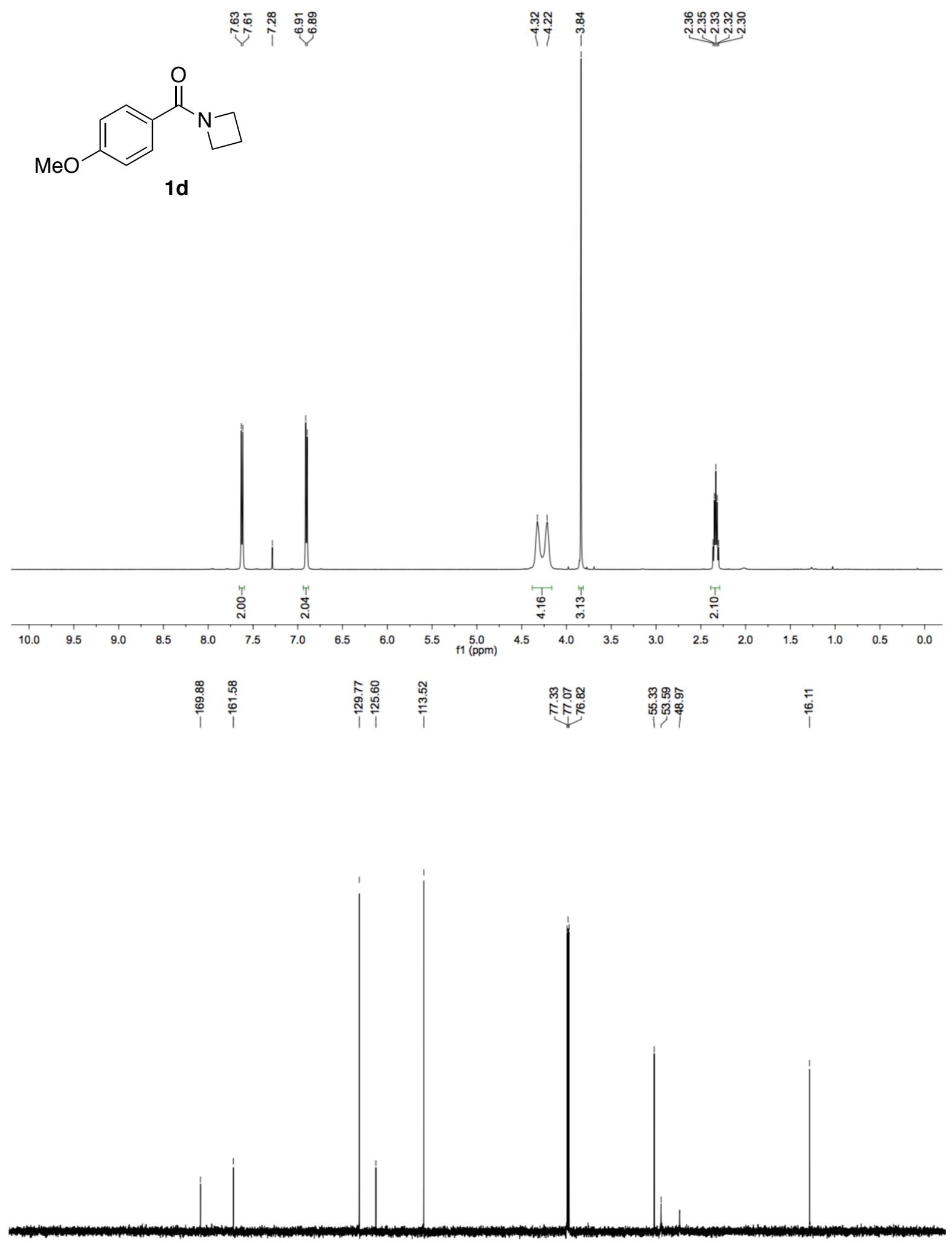

$\begin{array}{llllllllllllllllllllllllll}210 & 200 & 190 & 180 & 170 & 160 & 150 & 140 & 130 & 120 & 110 & 100 & 90 & 80 & 70 & 60 & 50 & 40 & 30 & 20 & 10 & 0 & -10\end{array}$ 


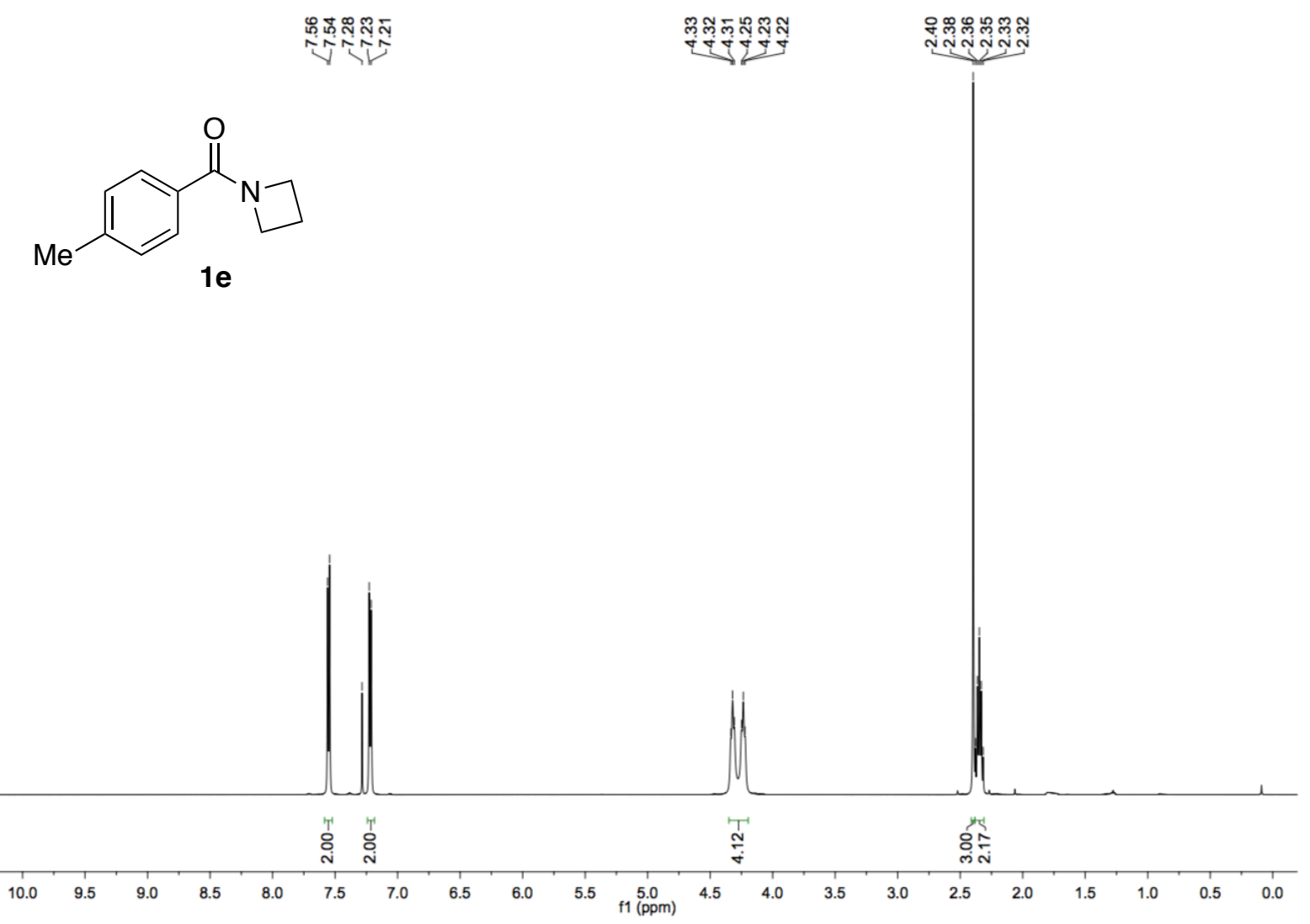

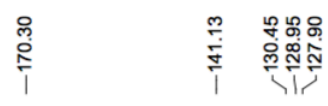
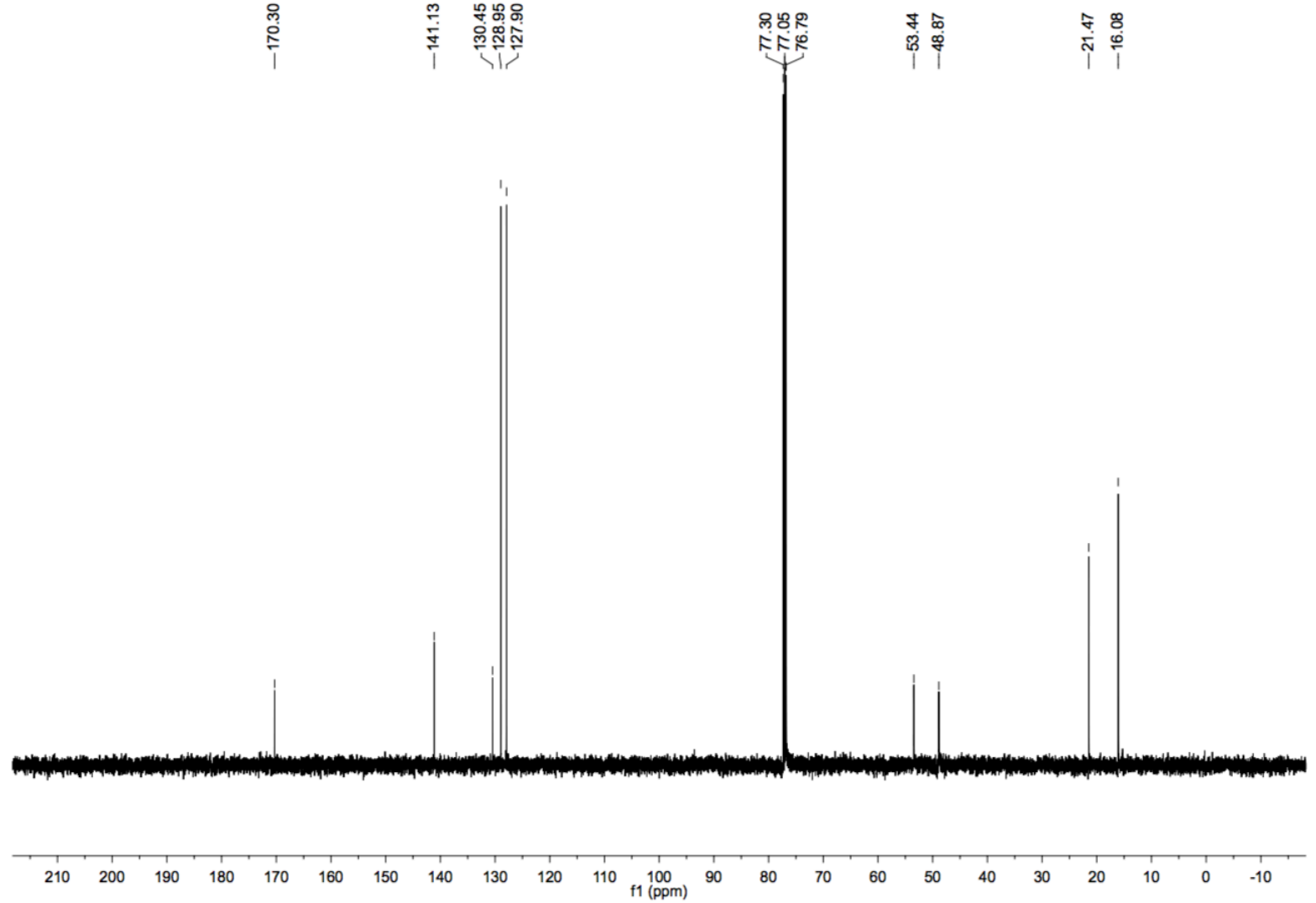

SI-30 

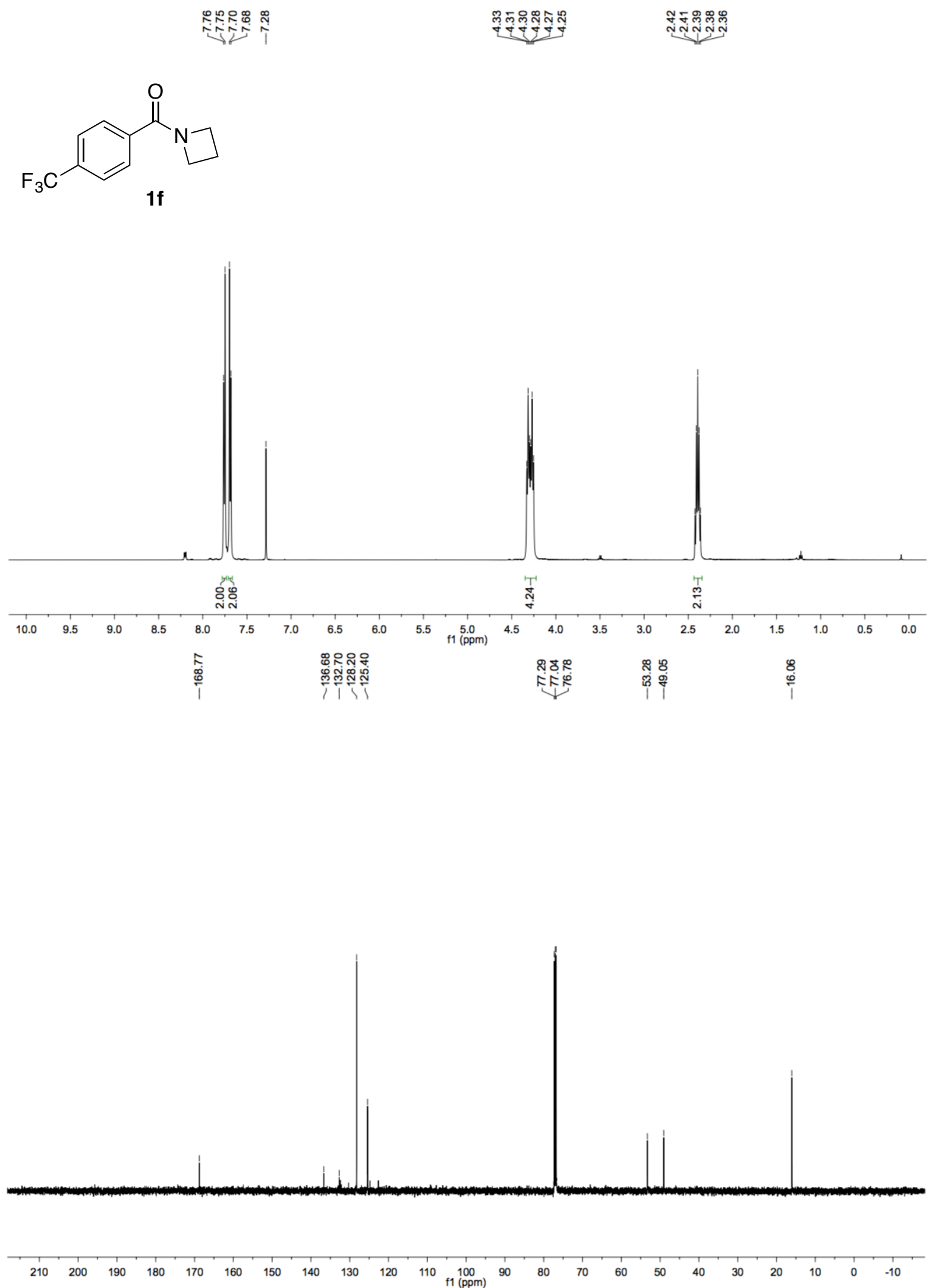

SI-31 


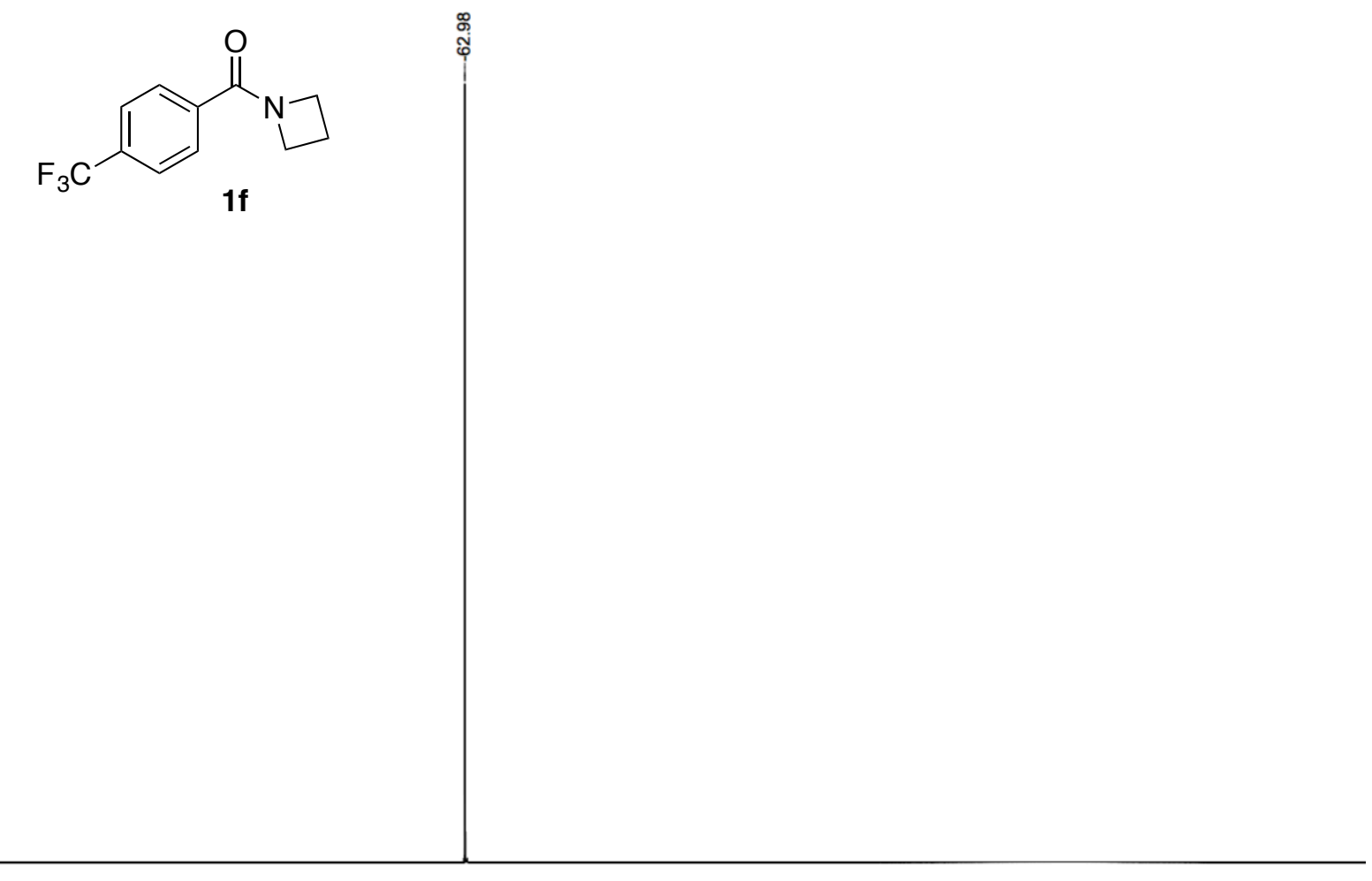

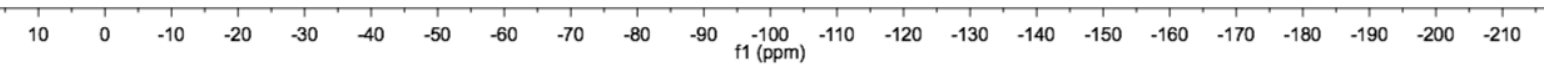


<smiles>O=C(c1ccc(F)cc1)N1CCC1</smiles>
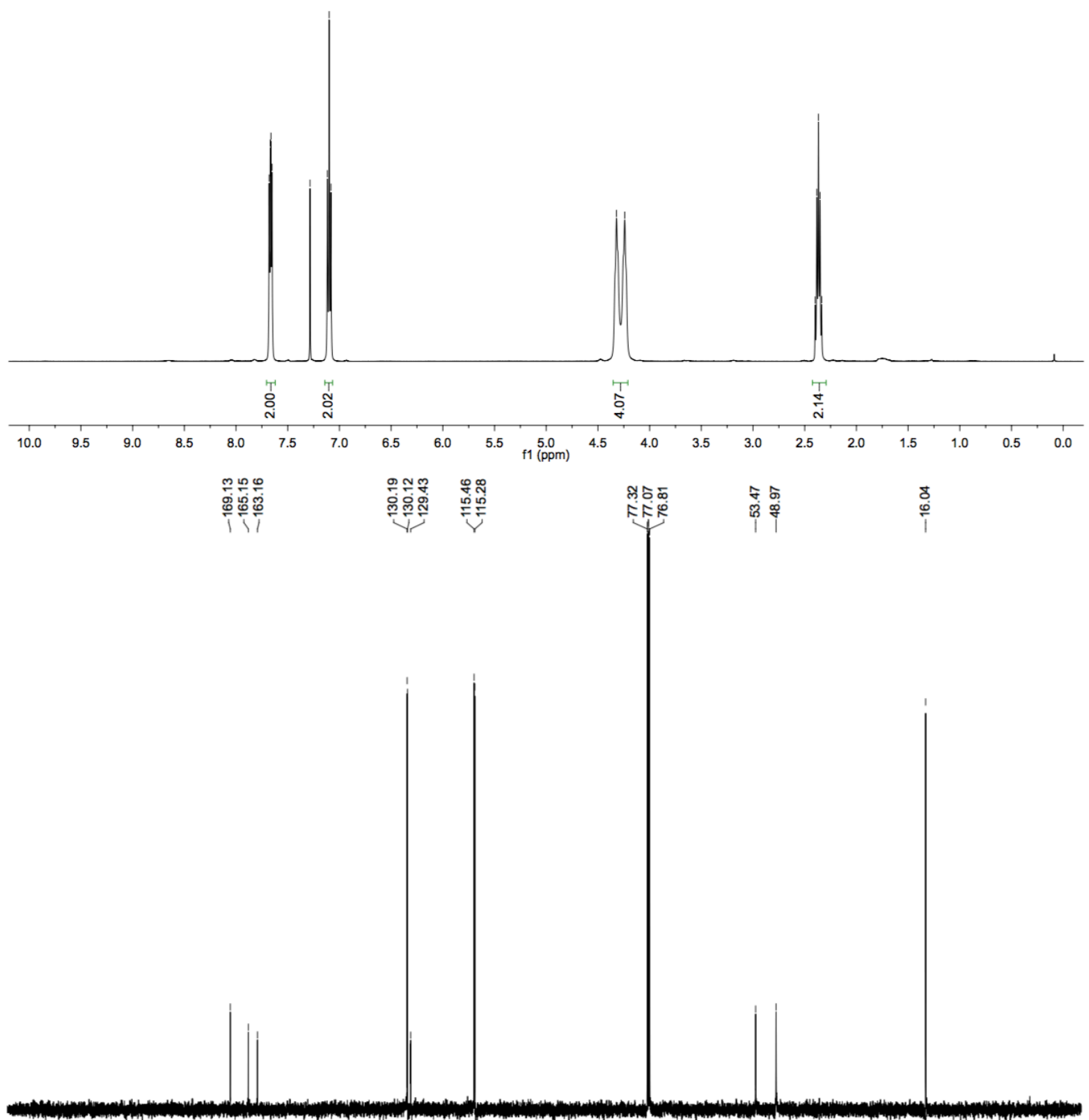

$\begin{array}{lllllllllllllllllllllll}210 & 200 & 190 & 180 & 170 & 160 & 150 & 140 & 130 & 120 & 110 & 100 & 90 & 80 & 70 & 60 & 50 & 40 & 30 & 20 & 10 & 0 & -10\end{array}$ 
<smiles>O=C(c1ccc(F)cc1)N1CCC1</smiles>

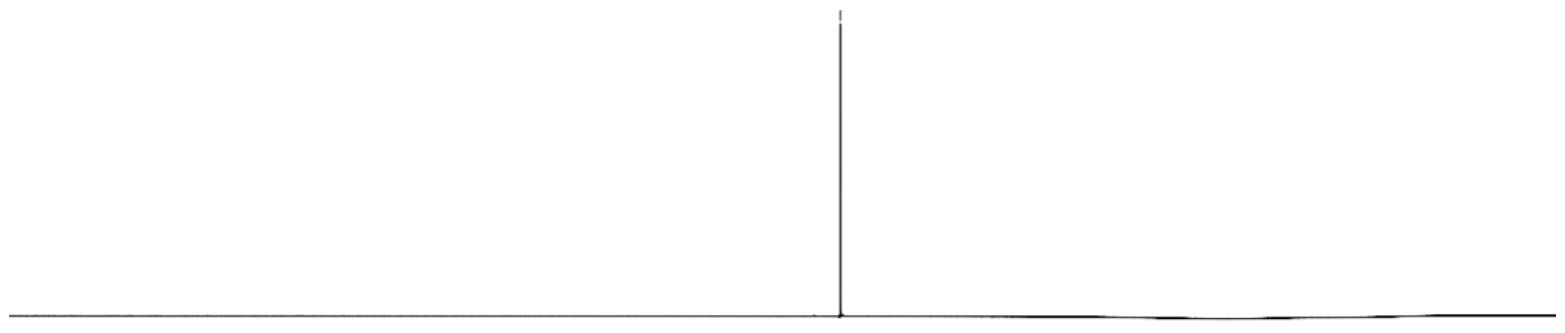

\begin{tabular}{llllllllllllllllllllllll}
\hline & 10 & 0 & -10 & -20 & -30 & -40 & -50 & -60 & -70 & -80 & -90 & -100 & -110 & -120 & -130 & -140 & -150 & -160 & -170 & -180 & -190 & -200 & -210
\end{tabular}



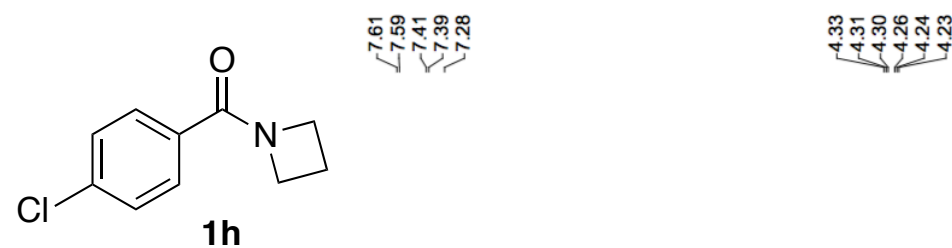

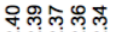

过开过

$1 \mathrm{~h}$
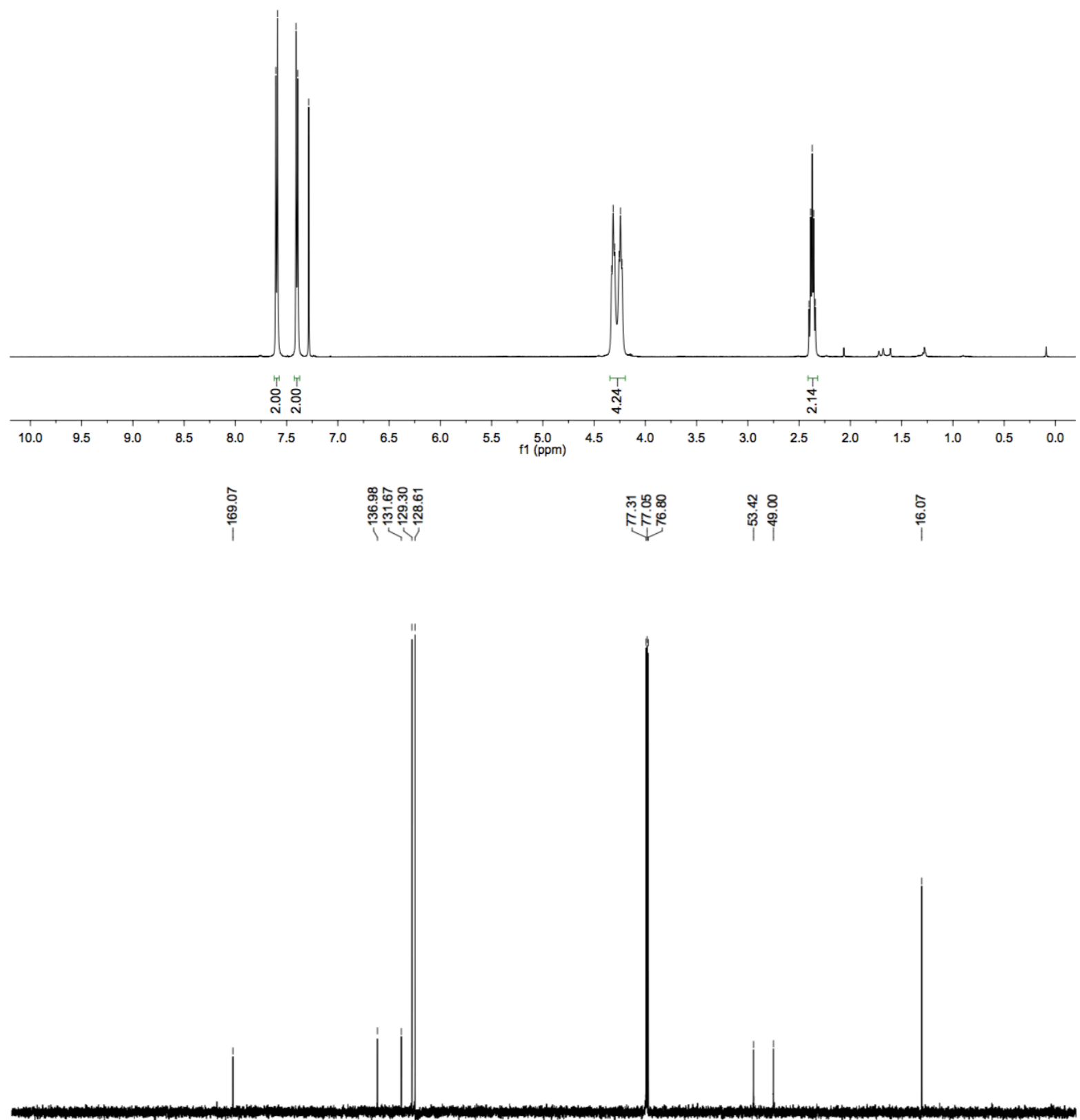

$\begin{array}{lllllllllllllllllllllll}210 & 200 & 190 & 180 & 170 & 160 & 150 & 140 & 130 & 120 & 110 & \begin{array}{c}100 \\ \mathrm{f} 1(\mathrm{ppm})\end{array} & 90 & 80 & 70 & 60 & 50 & 40 & 30 & 20 & 10 & 0 & -10\end{array}$

SI-35 


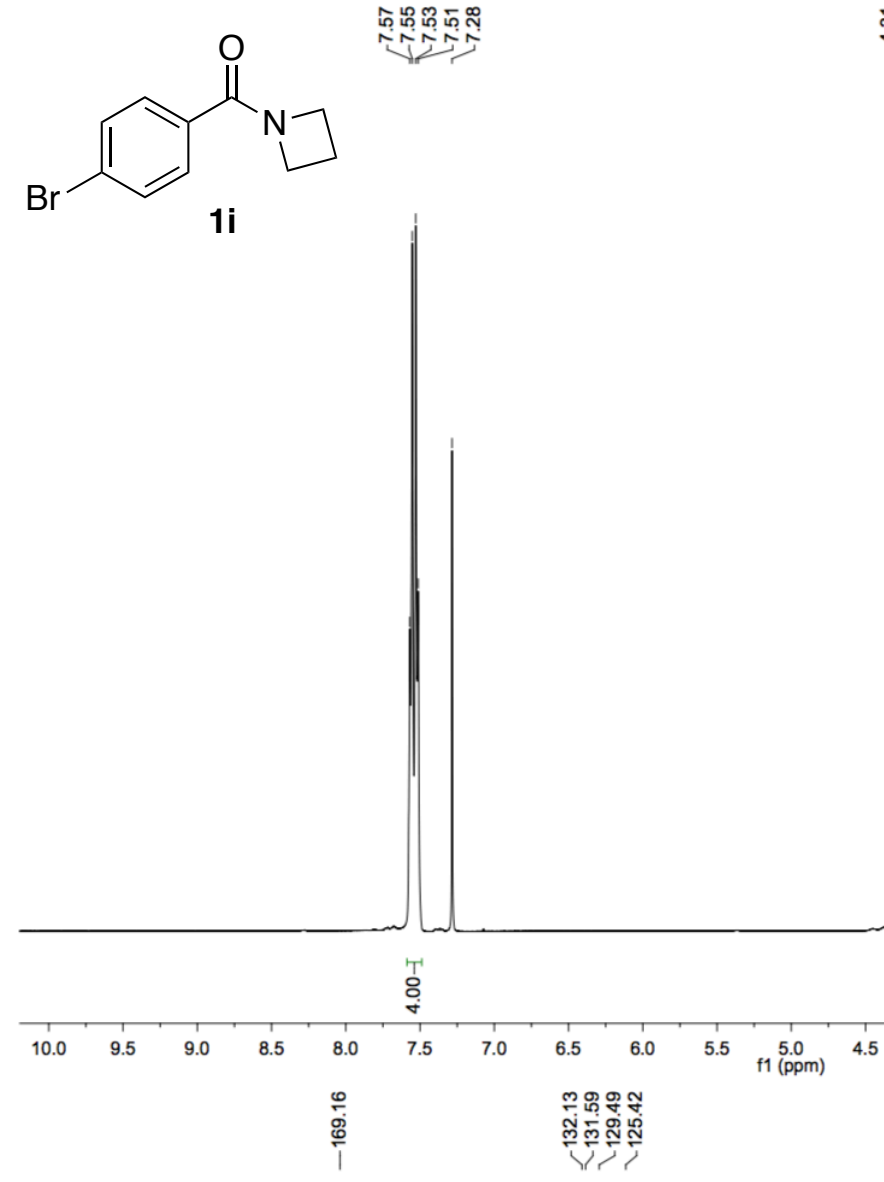

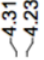

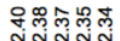

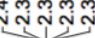

$\mathbf{B r}$
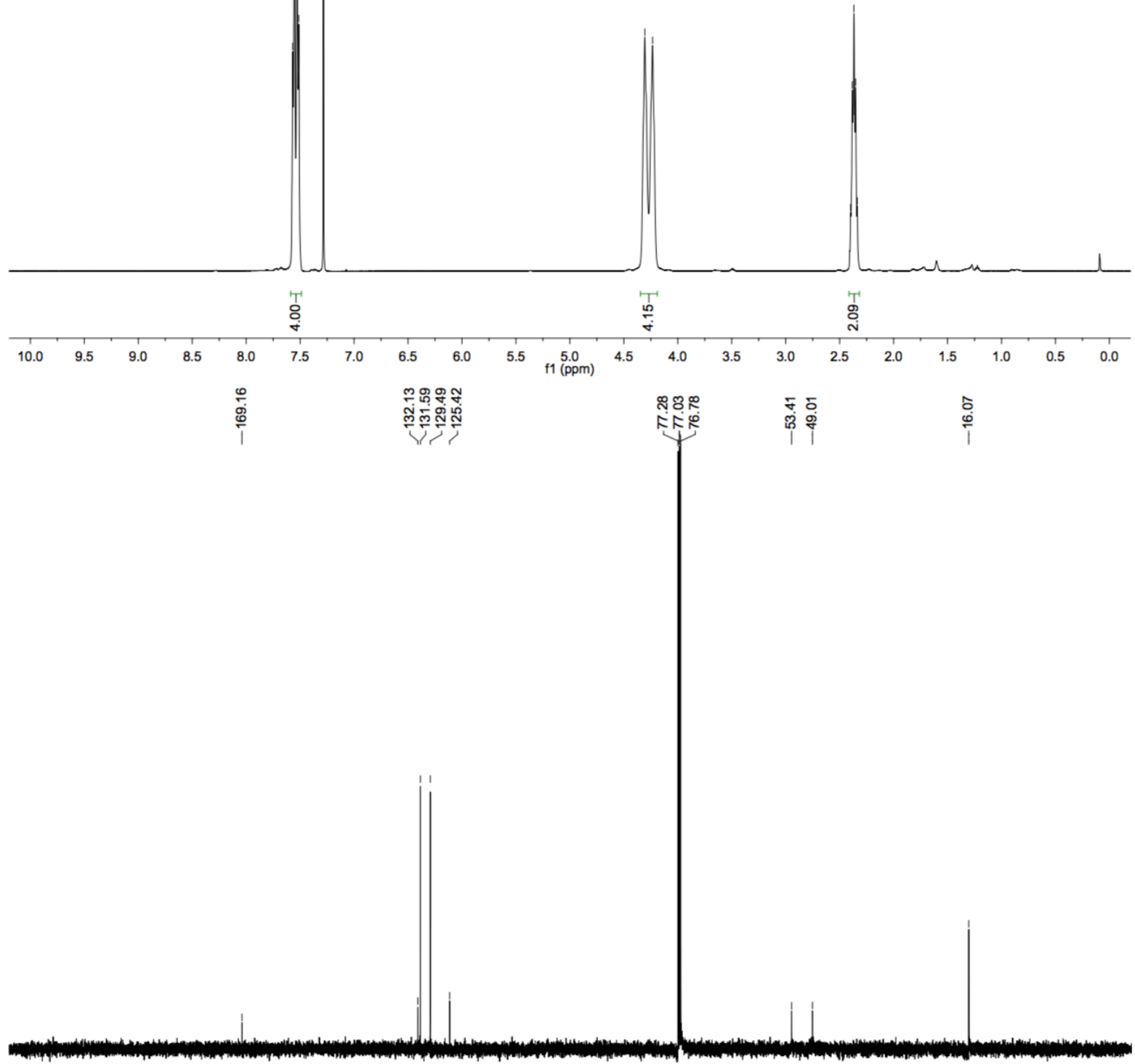

$\begin{array}{llllllllllllllllllllllllll}210 & 200 & 190 & 180 & 170 & 160 & 150 & 140 & 130 & 120 & 110 & 100 & 90 & 80 & 70 & 60 & 50 & 40 & 30 & 20 & 10 & 0 & -10\end{array}$

SI-36 

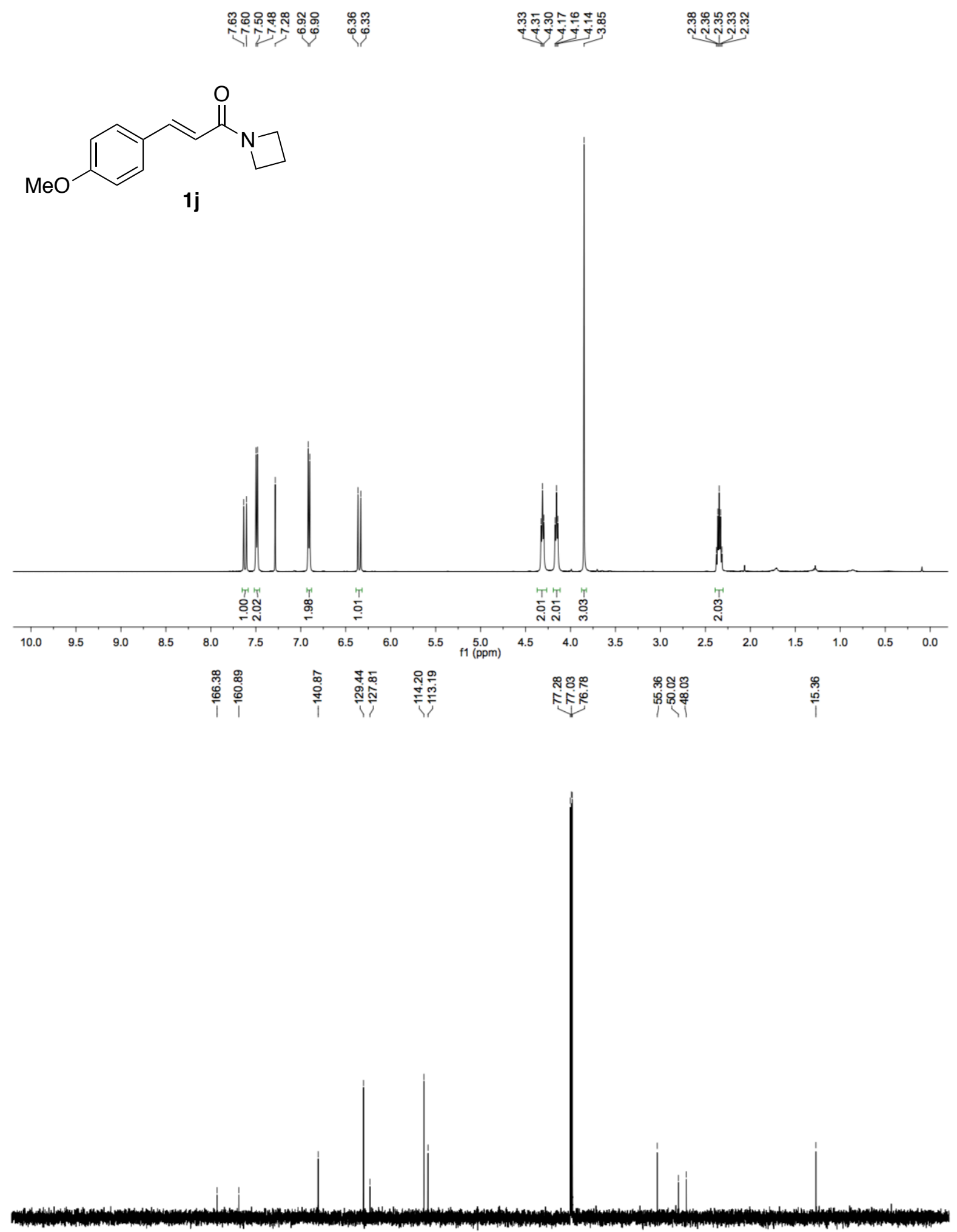

$\begin{array}{lllllllllllllllllllllllll}210 & 200 & 190 & 180 & 170 & 160 & 150 & 140 & 130 & 120 & 110 & 100 & 90 & 80 & 70 & 60 & 50 & 40 & 30 & 20 & 10 & 0 & -10\end{array}$ 


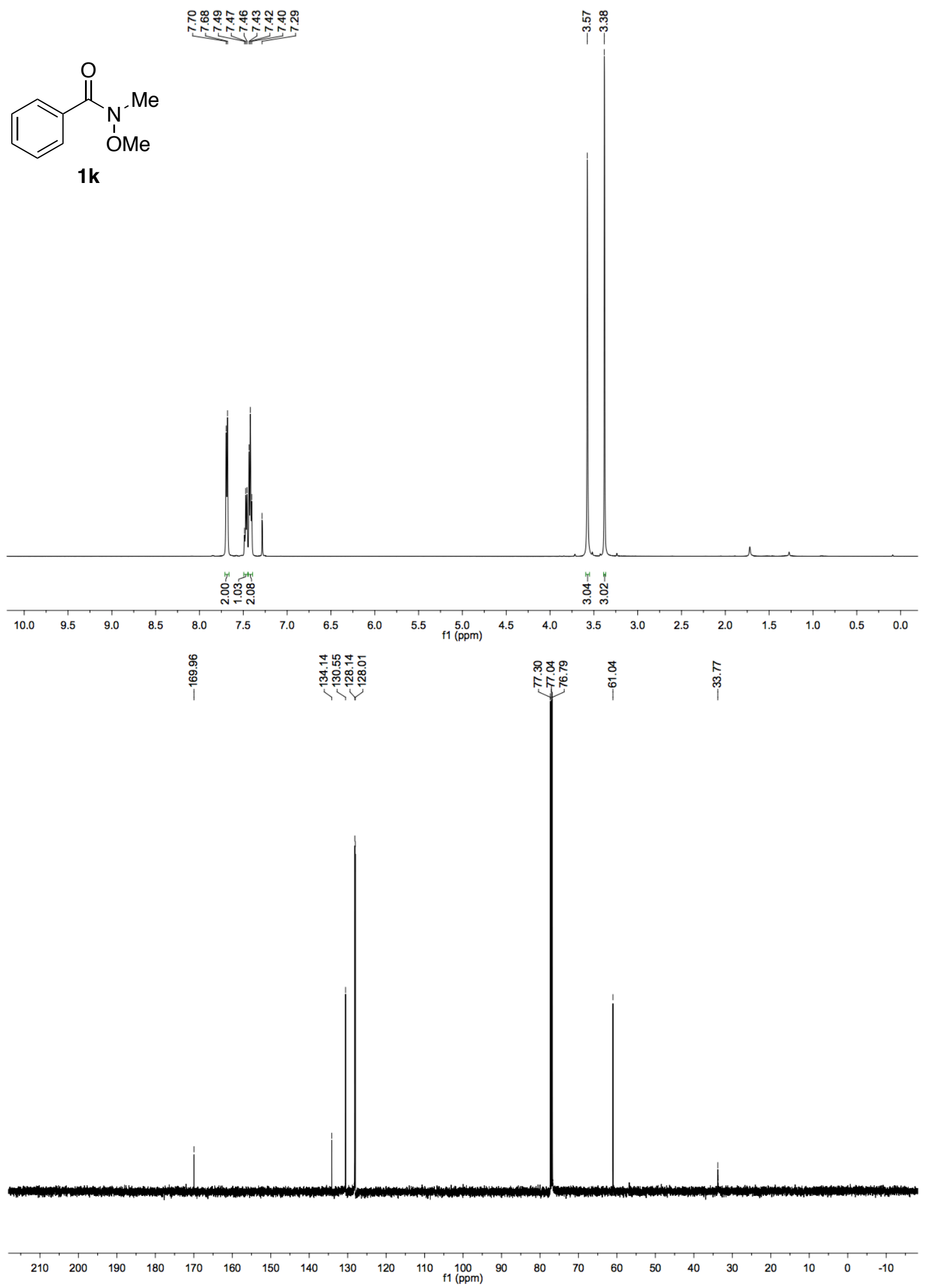

SI-38 

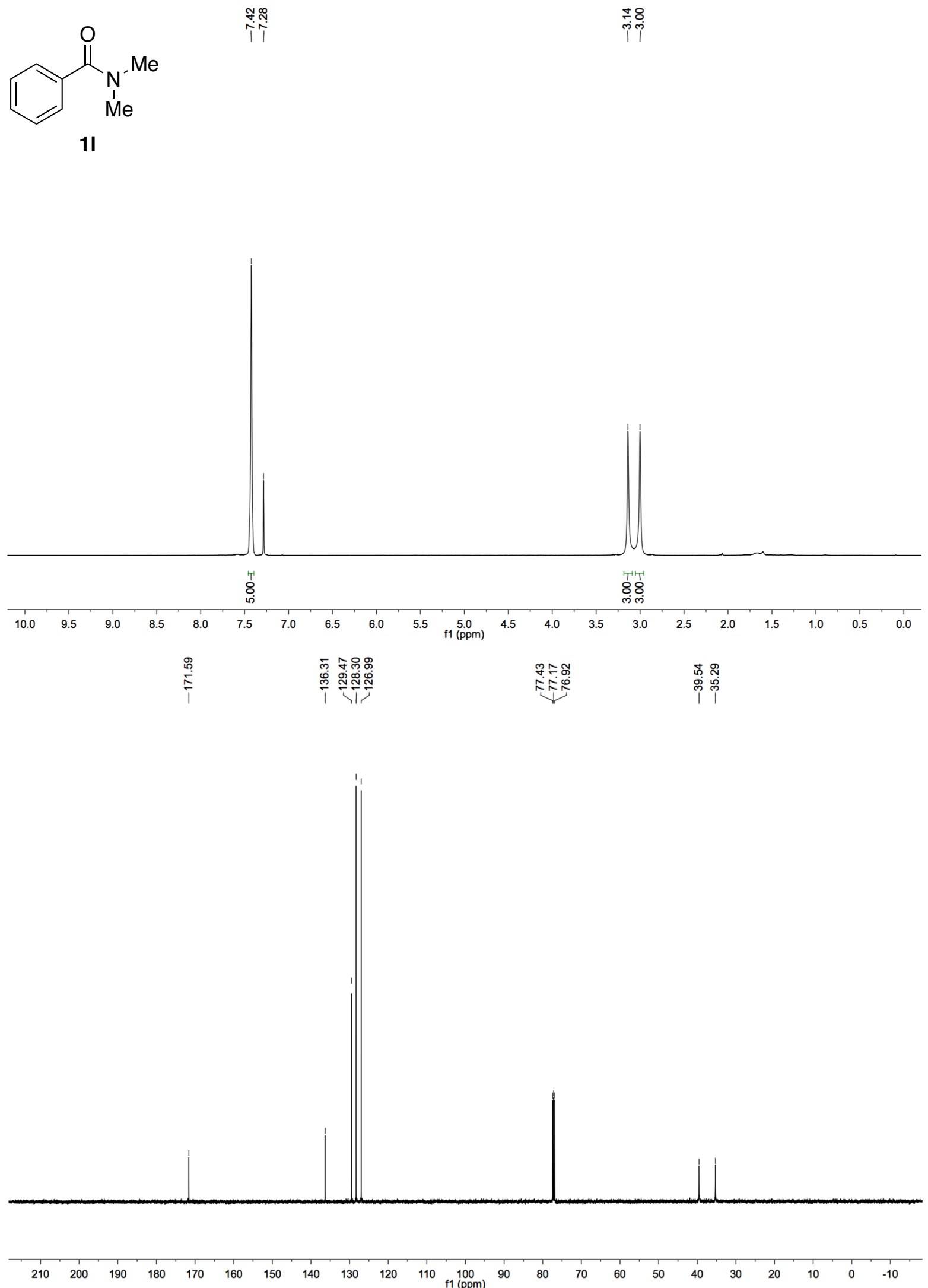

SI-39 

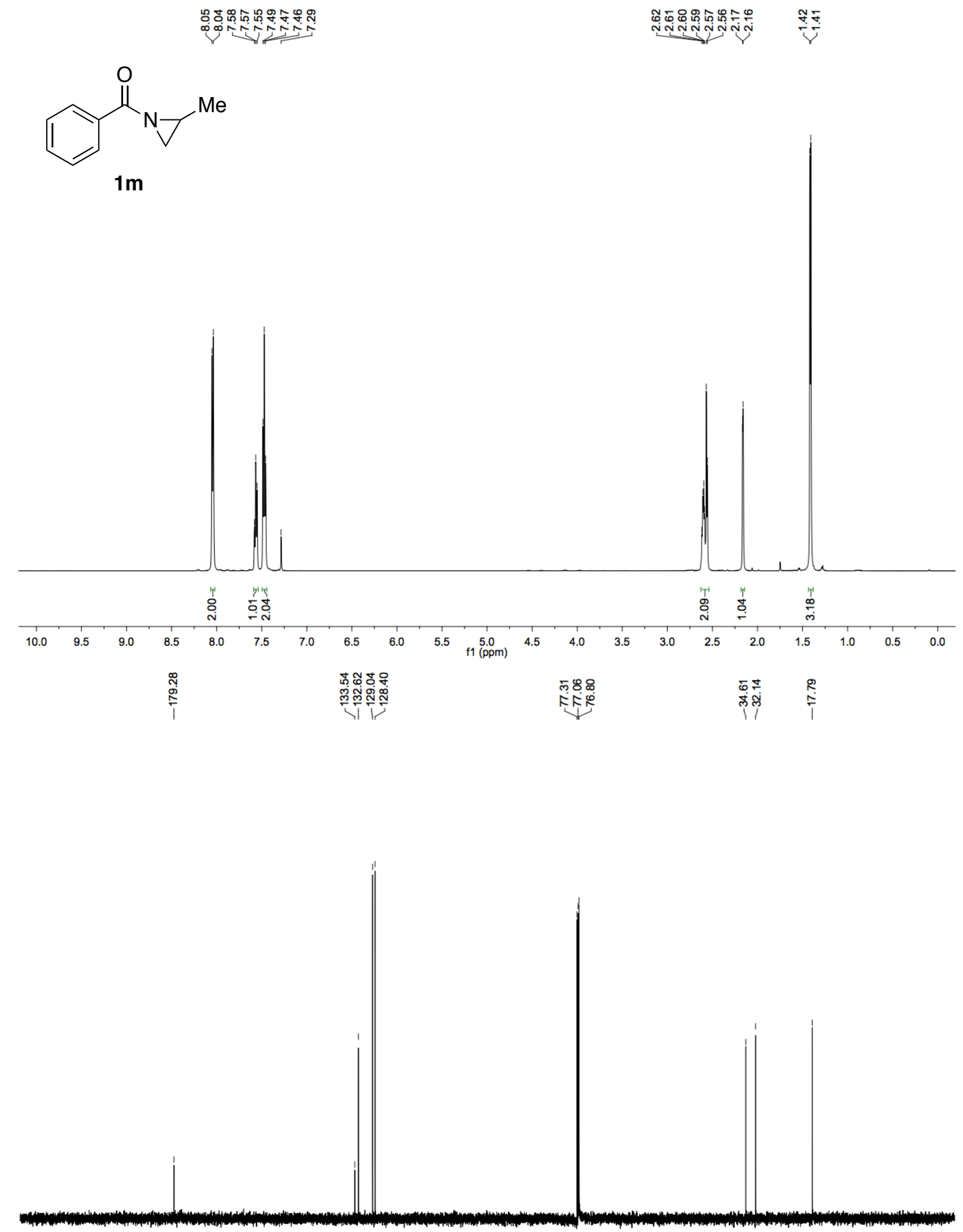

$\begin{array}{llllllllllllllllllllllllllllllll}210 & 200 & 190 & 180 & 170 & 160 & 150 & 140 & 130 & 120 & 110 & 100 & 90 & 80 & 70 & 60 & 50 & 40 & 30 & 20 & 10 & 0 & -10\end{array}$ 

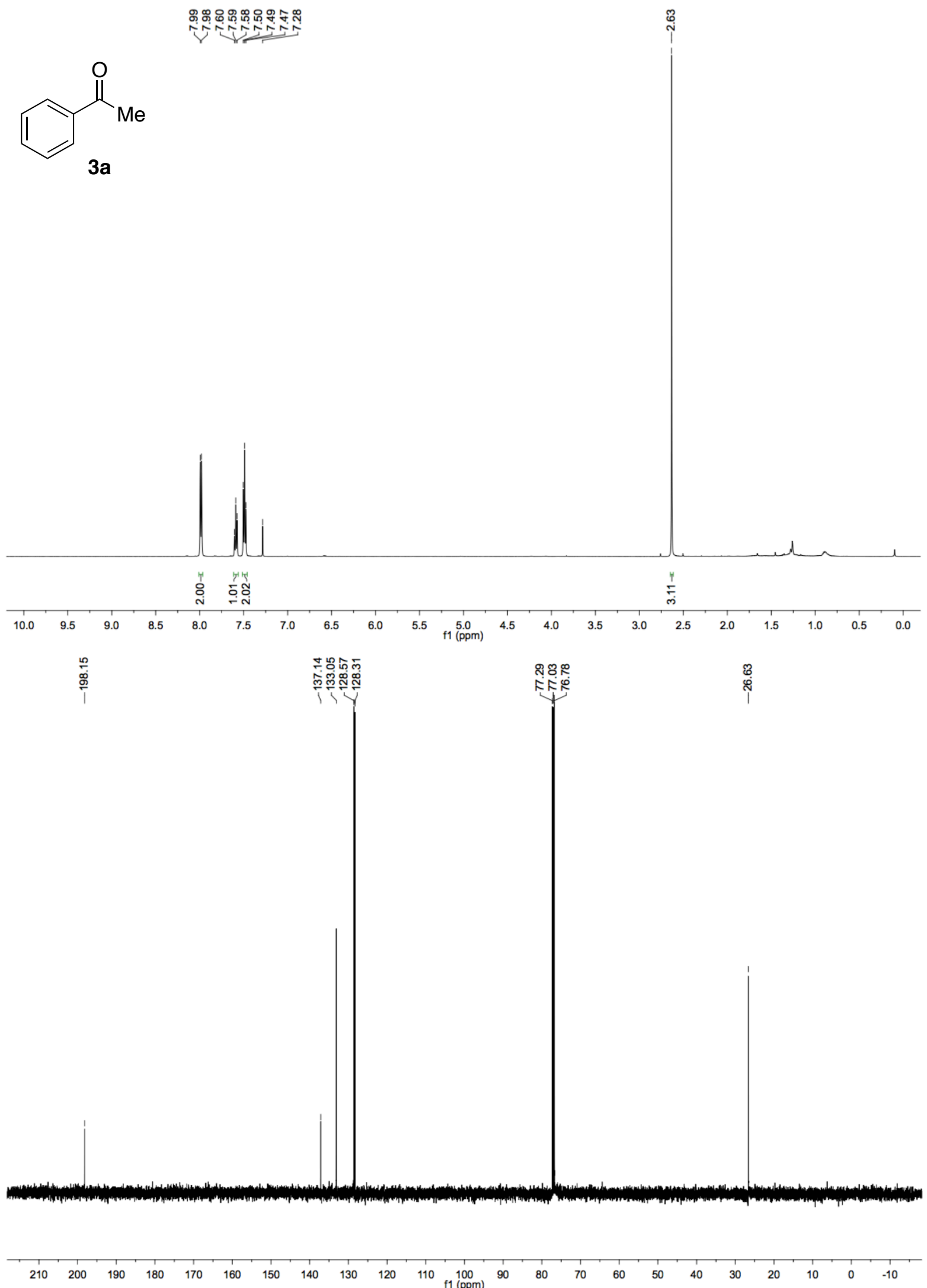

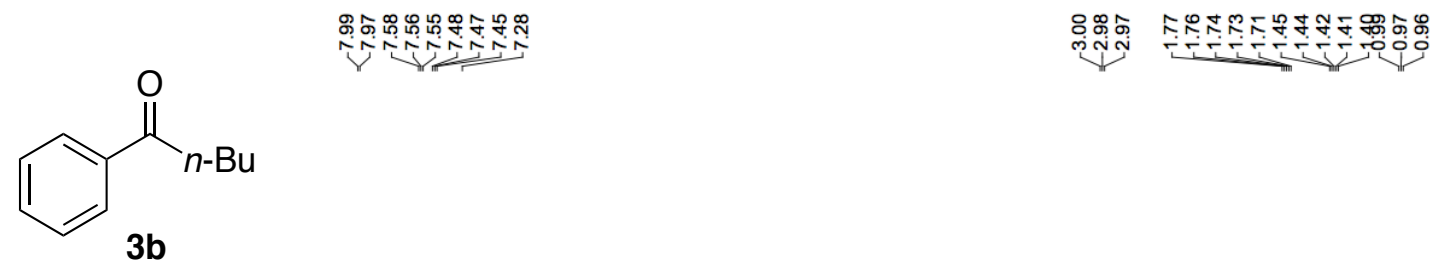

$3 b$
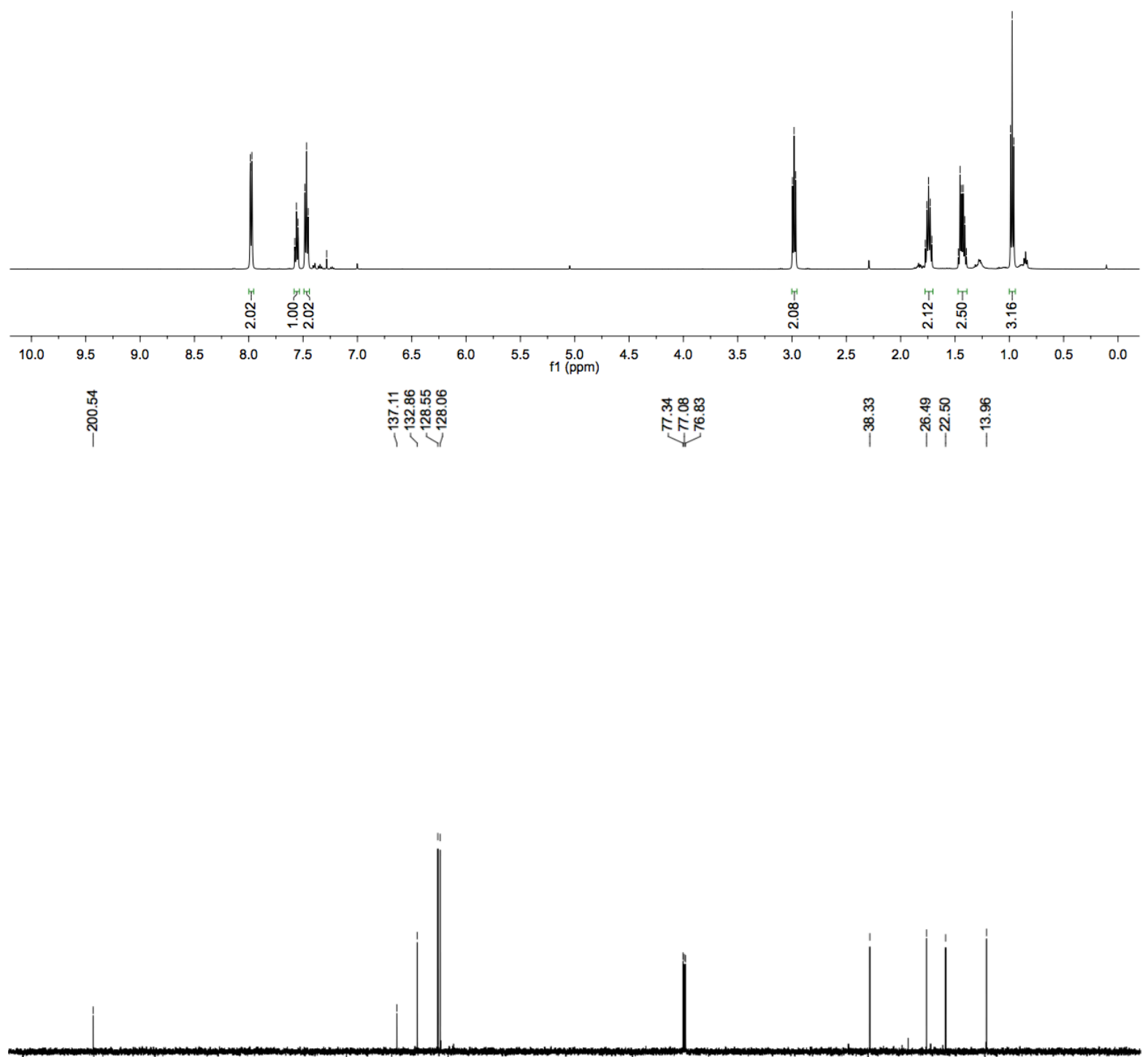

$\begin{array}{lllllllllllllllllllllllllll}210 & 200 & 190 & 180 & 170 & 160 & 150 & 140 & 130 & 120 & 110 & 100 & 90 & 80 & 70 & 60 & 50 & 40 & 30 & 20 & 10 & 0 & -10\end{array}$ 

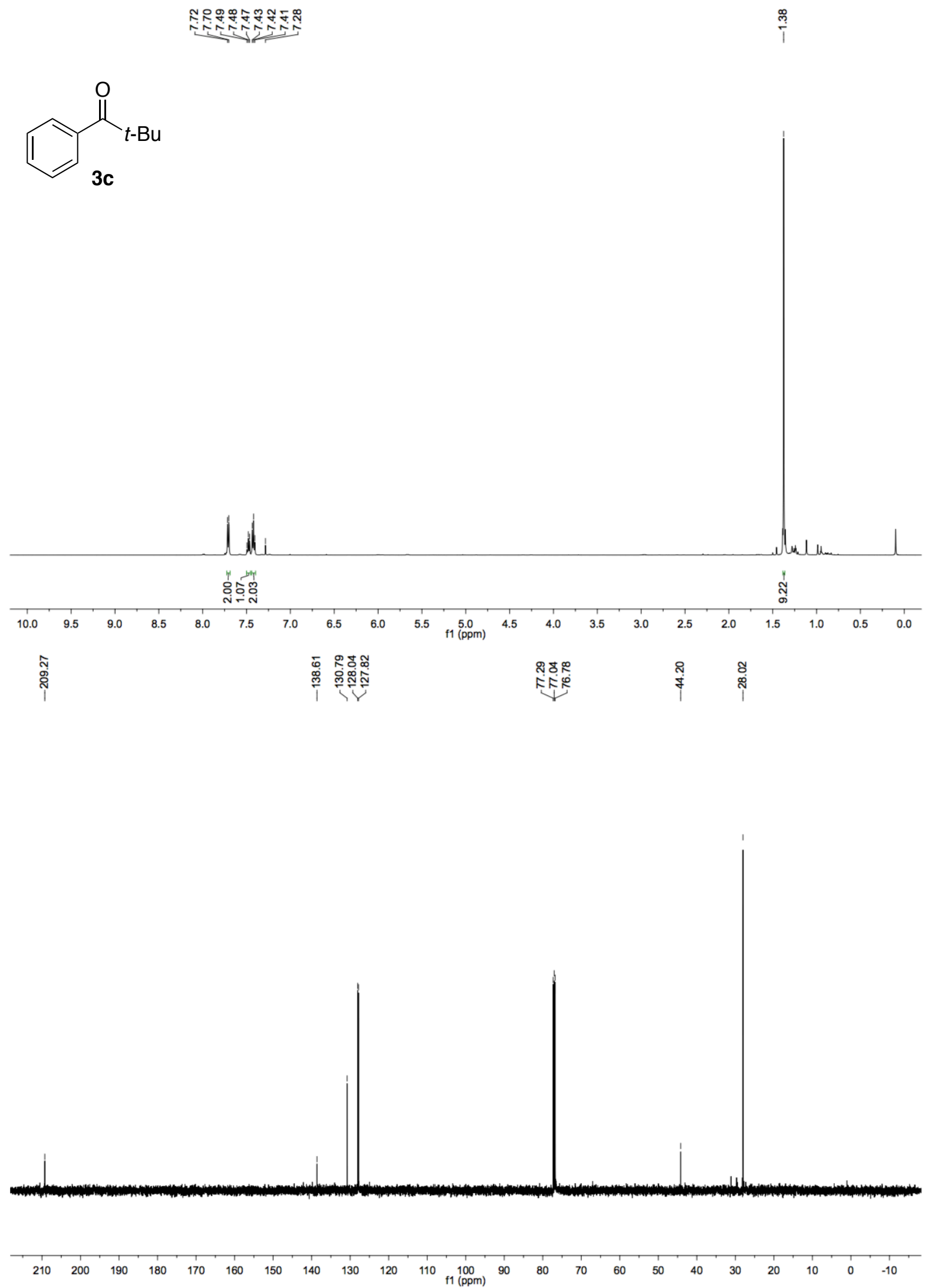

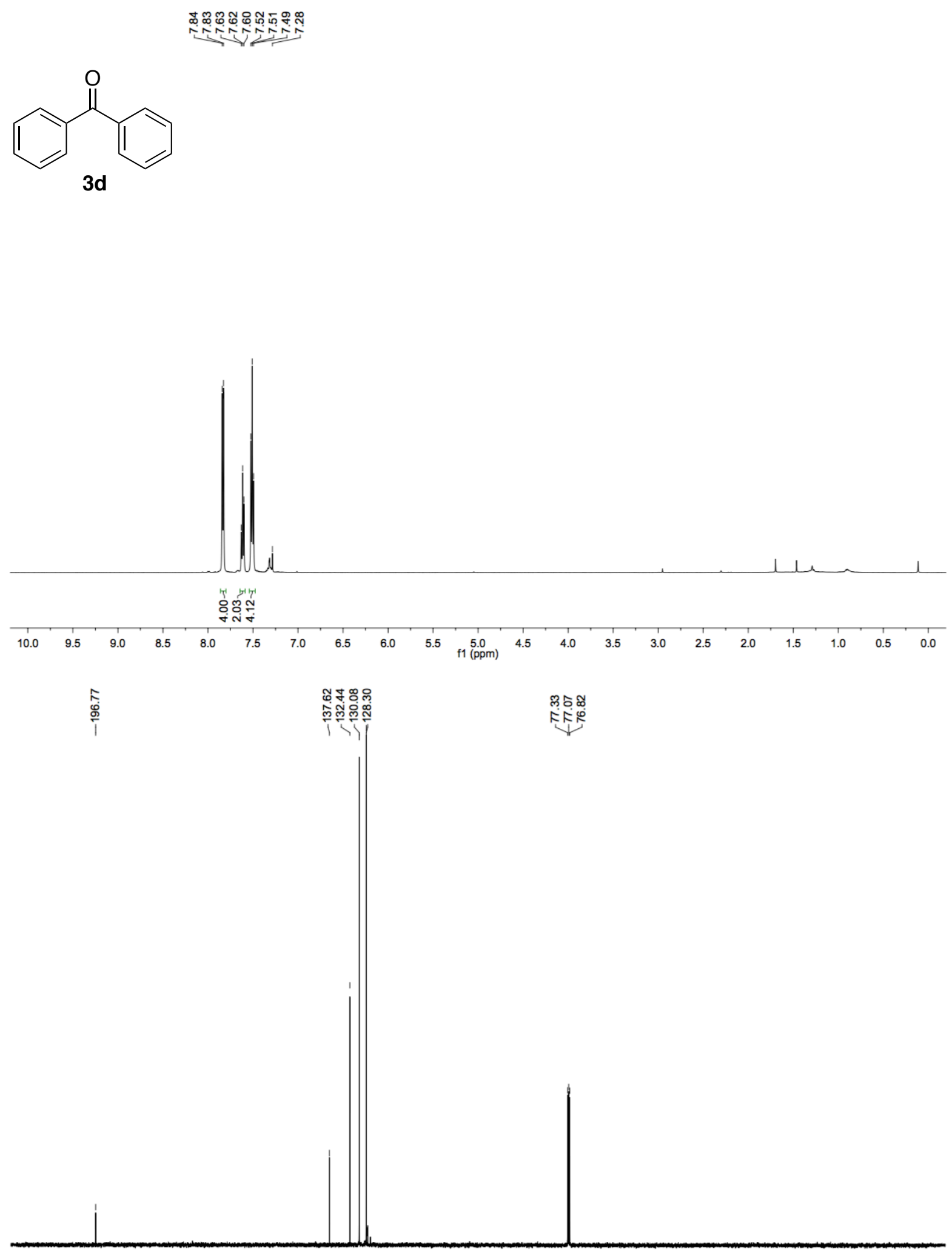

$\begin{array}{lllllllllllllllllllllllllll}210 & 200 & 190 & 180 & 170 & 160 & 150 & 140 & 130 & 120 & 110 & 100 & 90 & 80 & 70 & 60 & 50 & 40 & 30 & 20 & 10 & 0 & -10\end{array}$

SI-44 

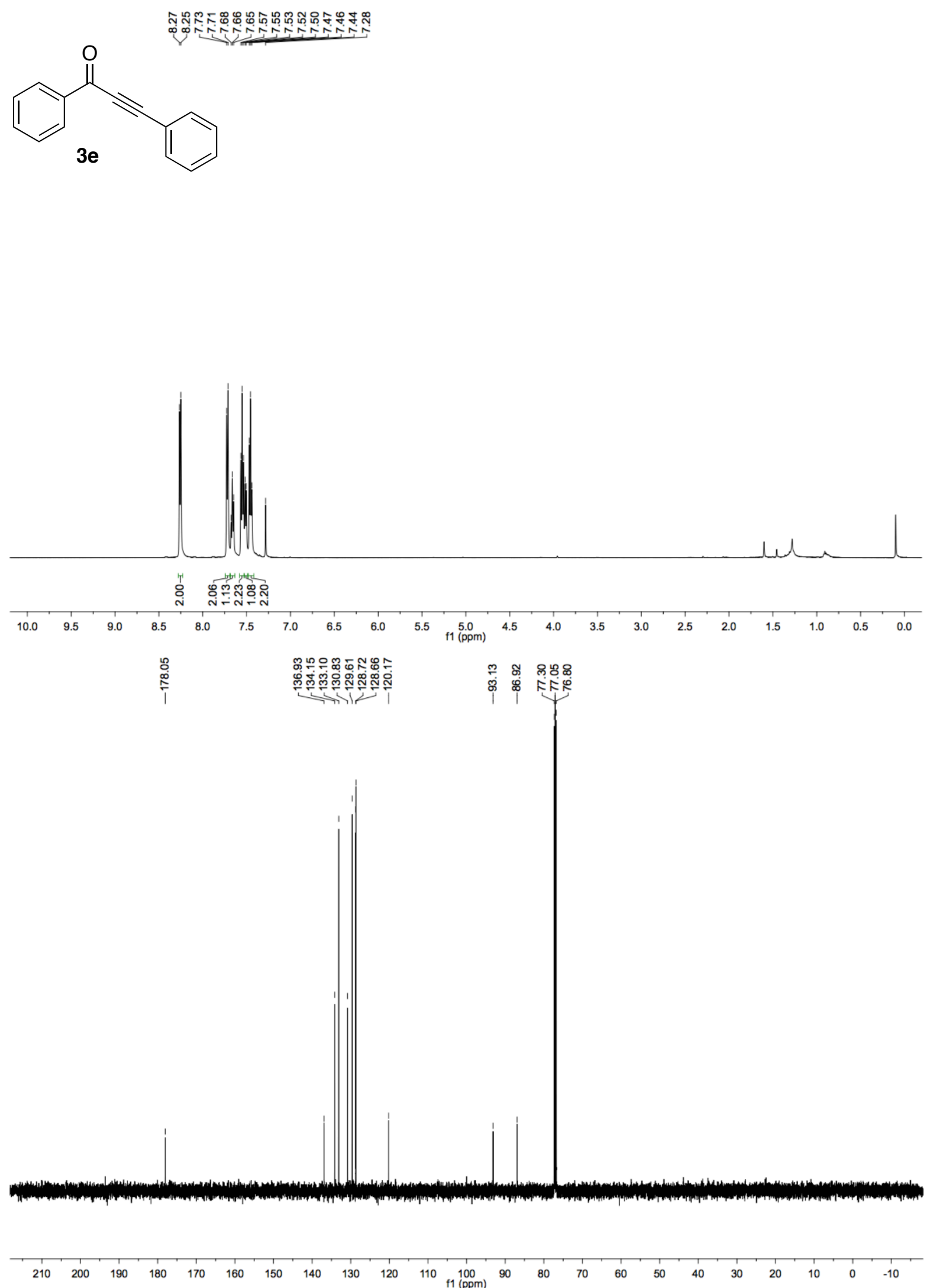


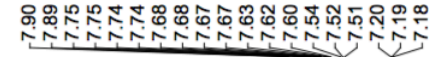<smiles>O=C(c1ccccc1)c1cccs1</smiles>

$3 f$
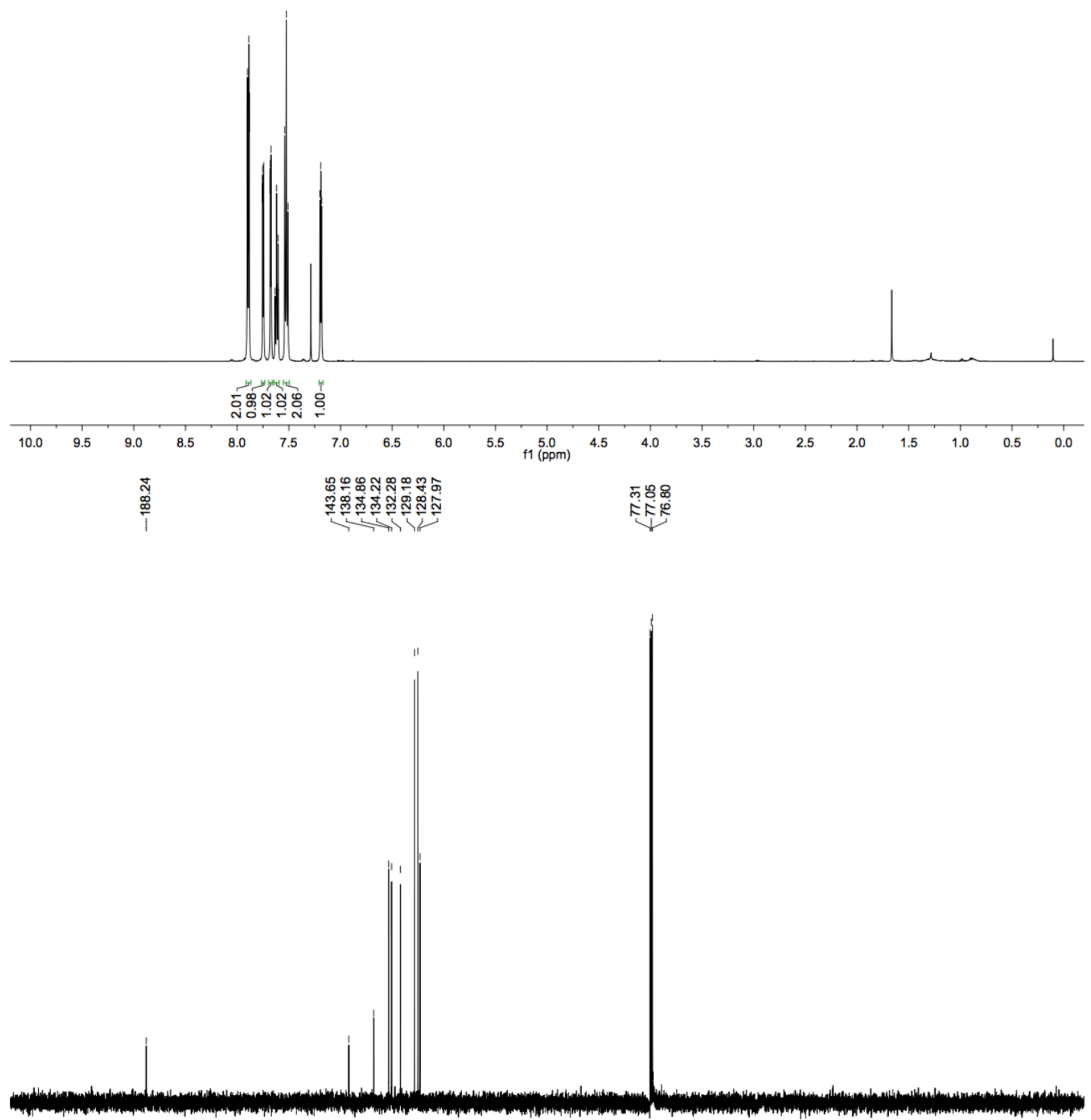

$\begin{array}{llllllllllllllllllllllll}210 & 200 & 190 & 180 & 170 & 160 & 150 & 140 & 130 & 120 & 110 & 100 & 90 & 80 & 70 & 60 & 50 & 40 & 30 & 20 & 10 & 0 & -10\end{array}$ 

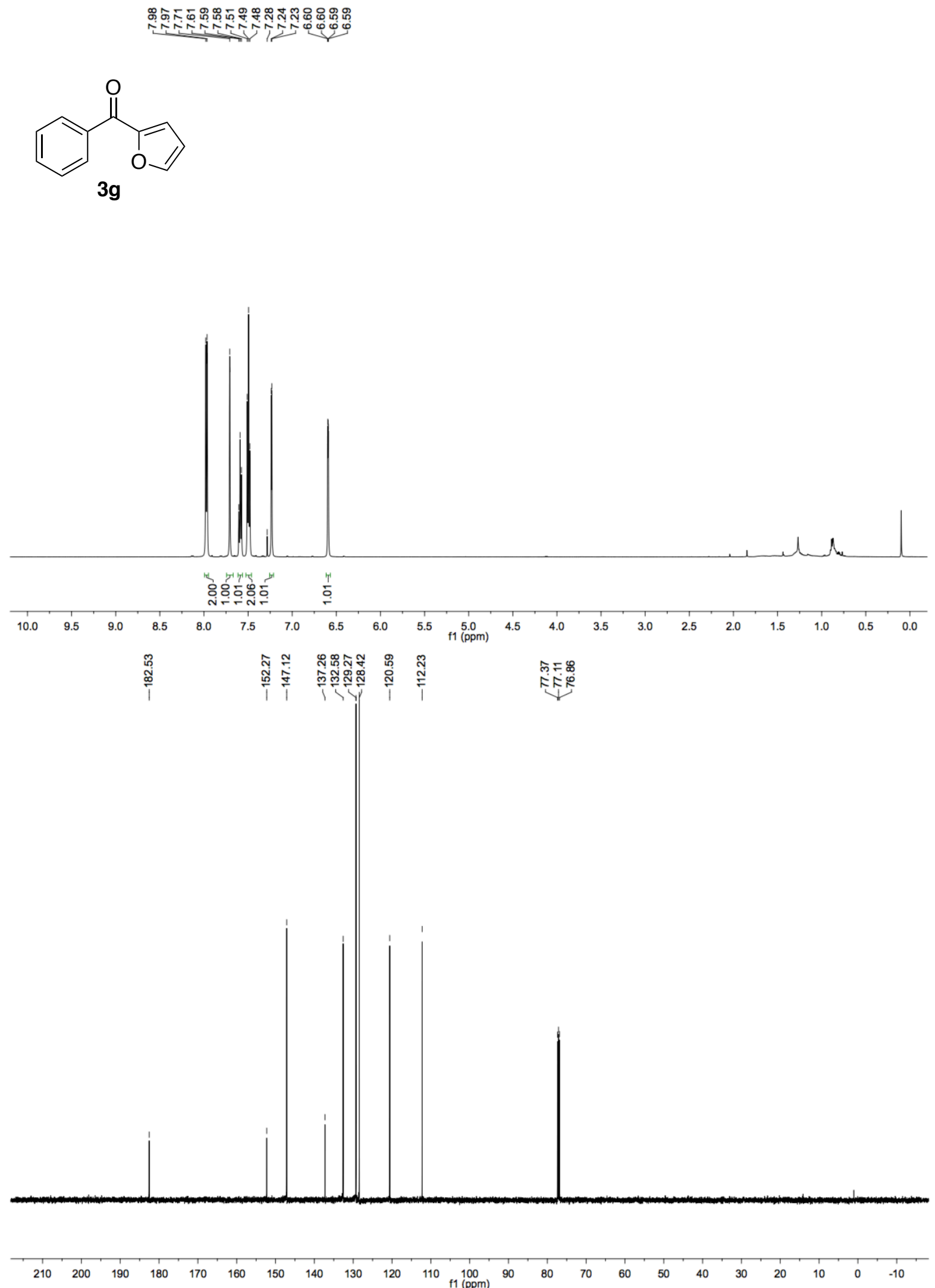


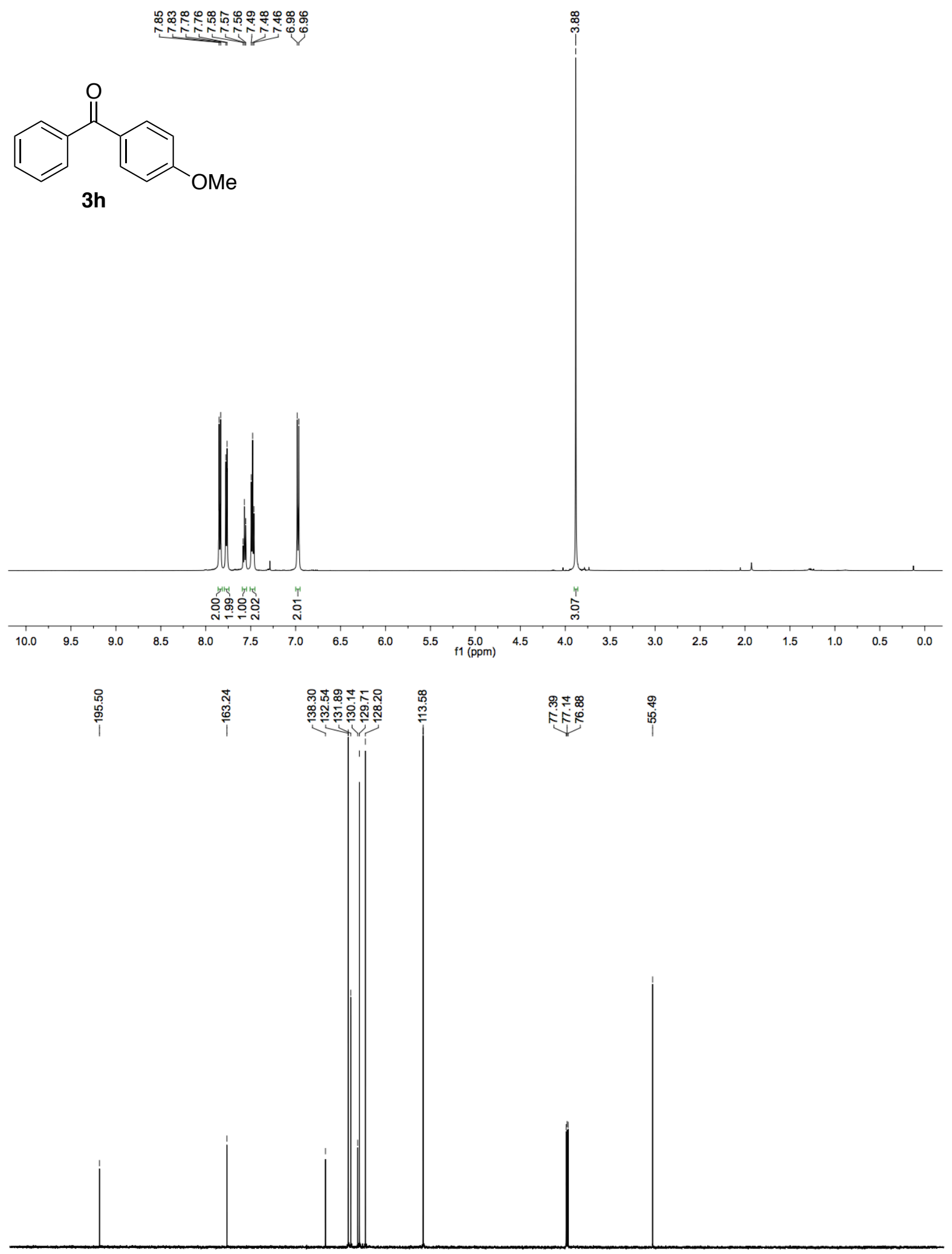

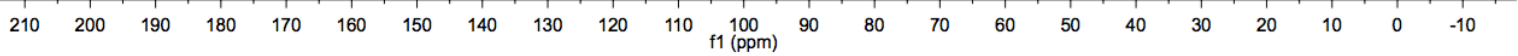



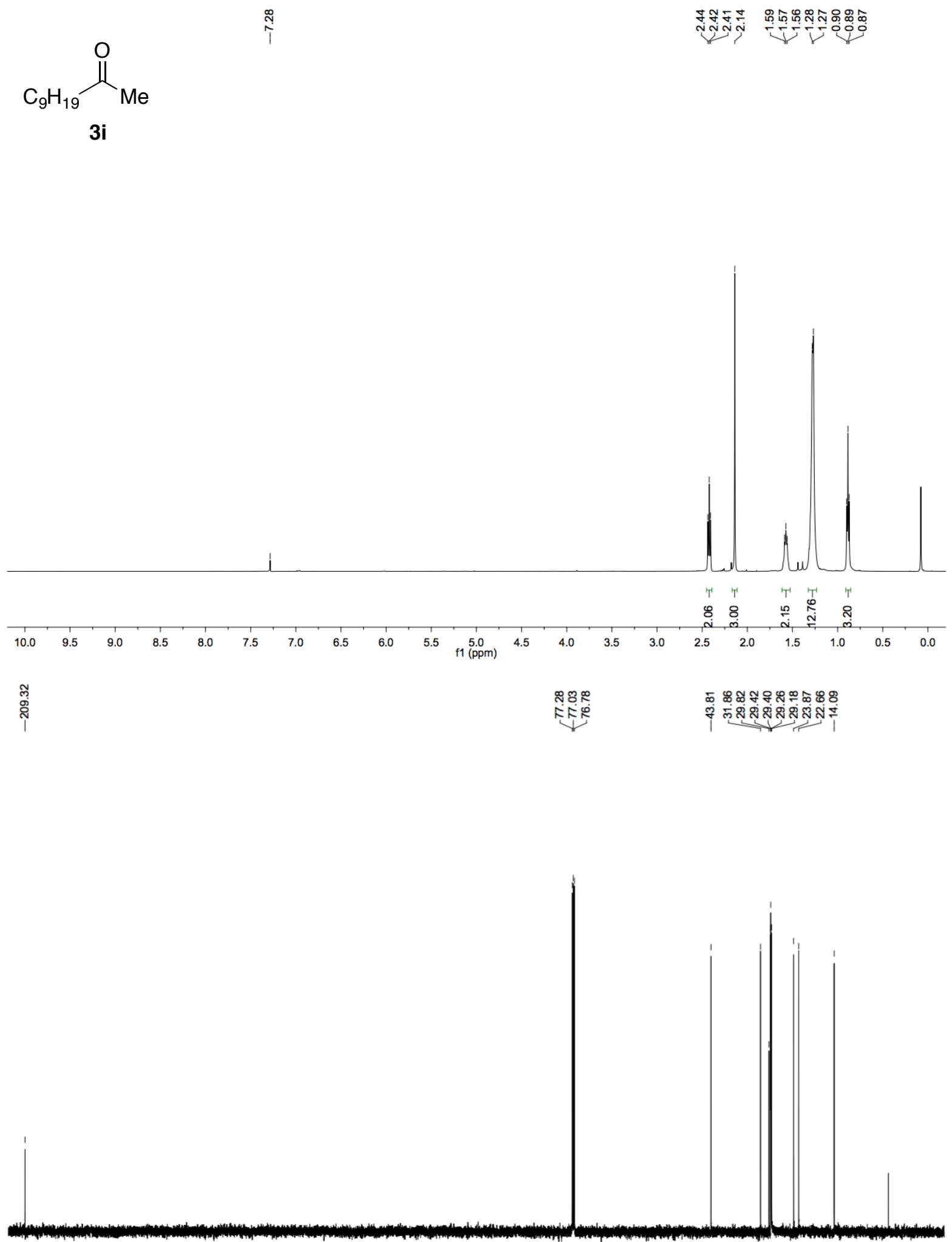

$\begin{array}{llllllllllllllllllllllllll}210 & 200 & 190 & 180 & 170 & 160 & 150 & 140 & 130 & 120 & 110 & 100 & 90 & 80 & 70 & 60 & 50 & 40 & 30 & 20 & 10 & 0 & -10\end{array}$ SI-49 
<smiles>CCCCCCCCCCCCCCCCCCCC</smiles>

3j

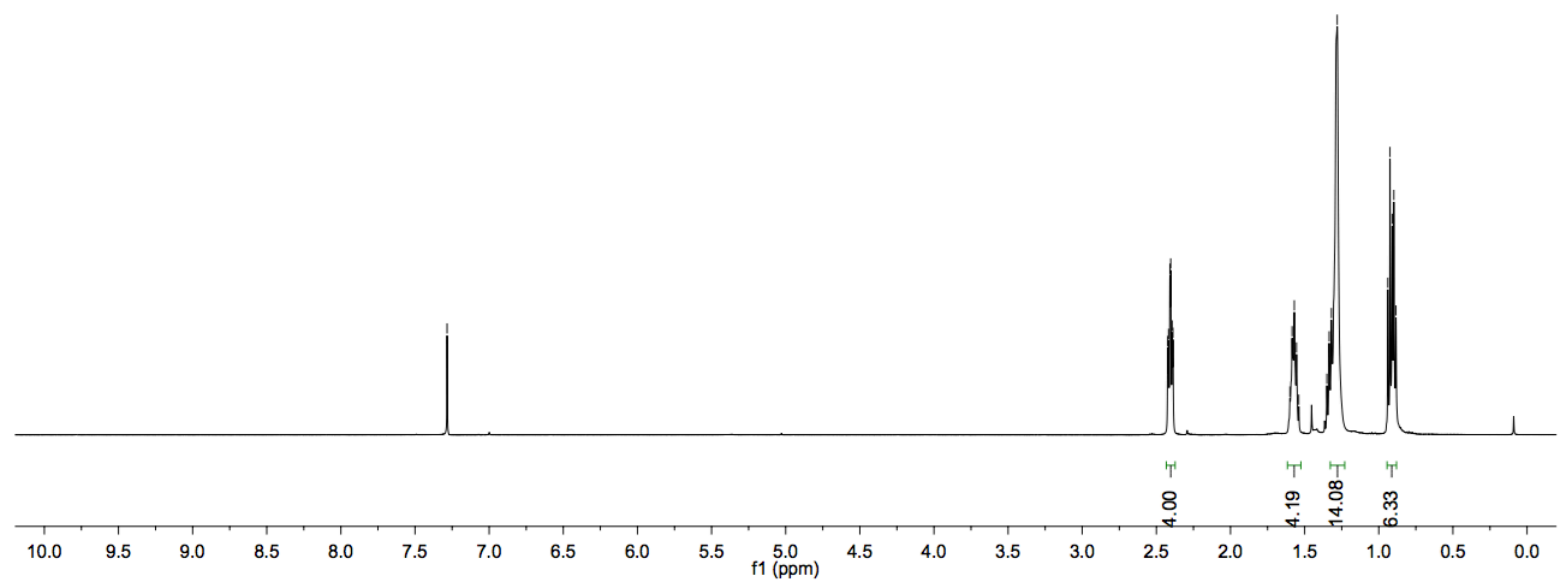

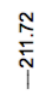

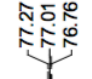

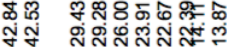

ปู่

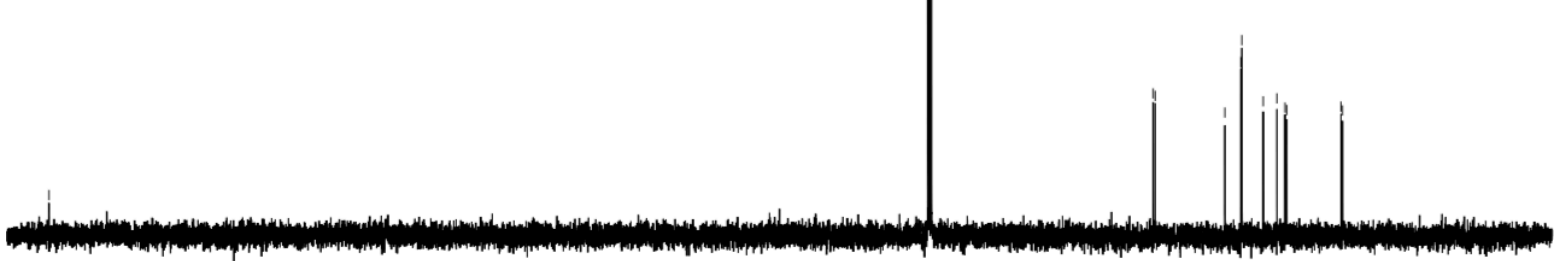

$\begin{array}{llllllllllll}210 & 200 & 190 & 180 & 170 & 160 & 150 & 140 & 130 & 120 & 110 & \begin{array}{l}100 \\ \mathrm{f} 1(\mathrm{ppm})\end{array}\end{array}$ 

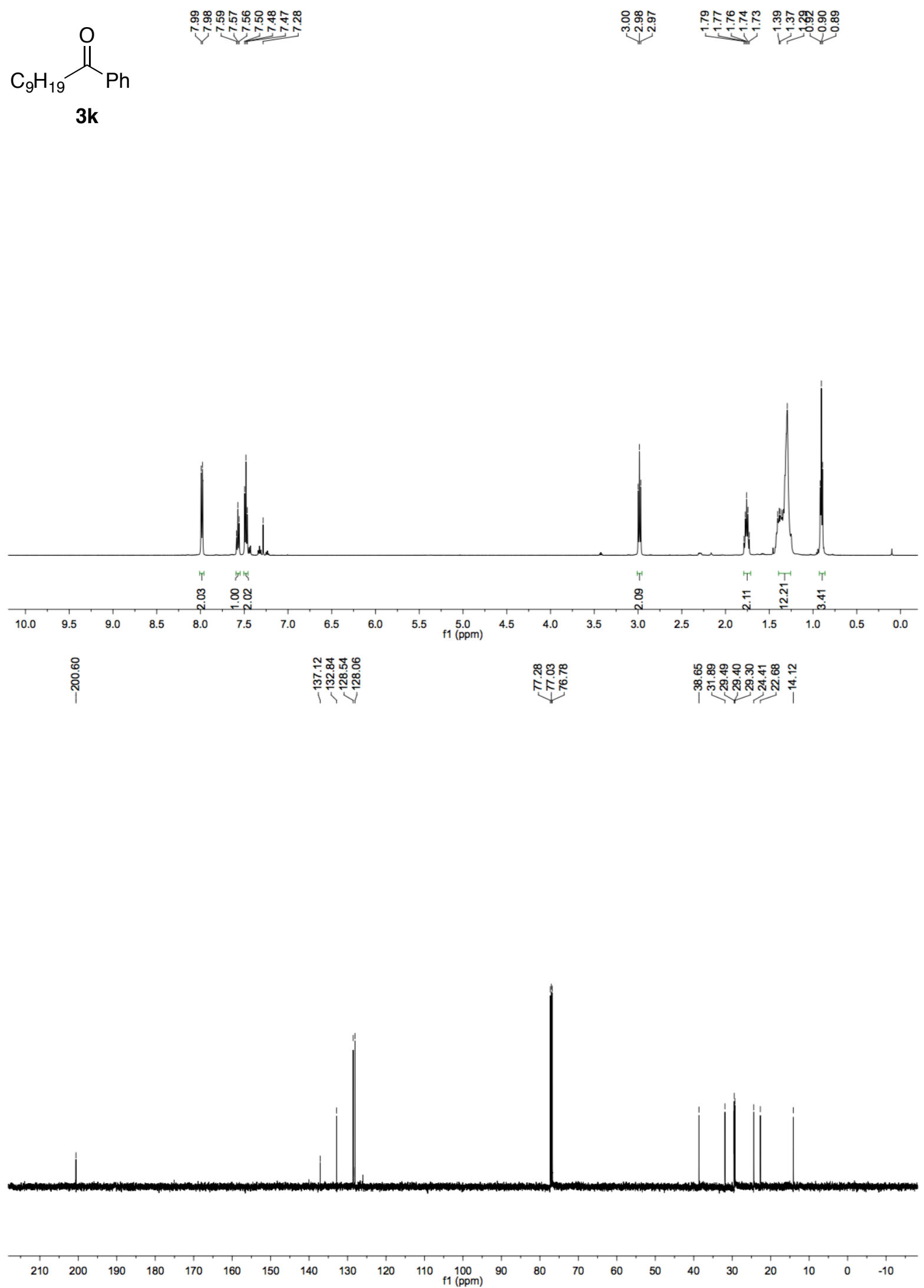


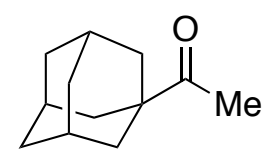

3!

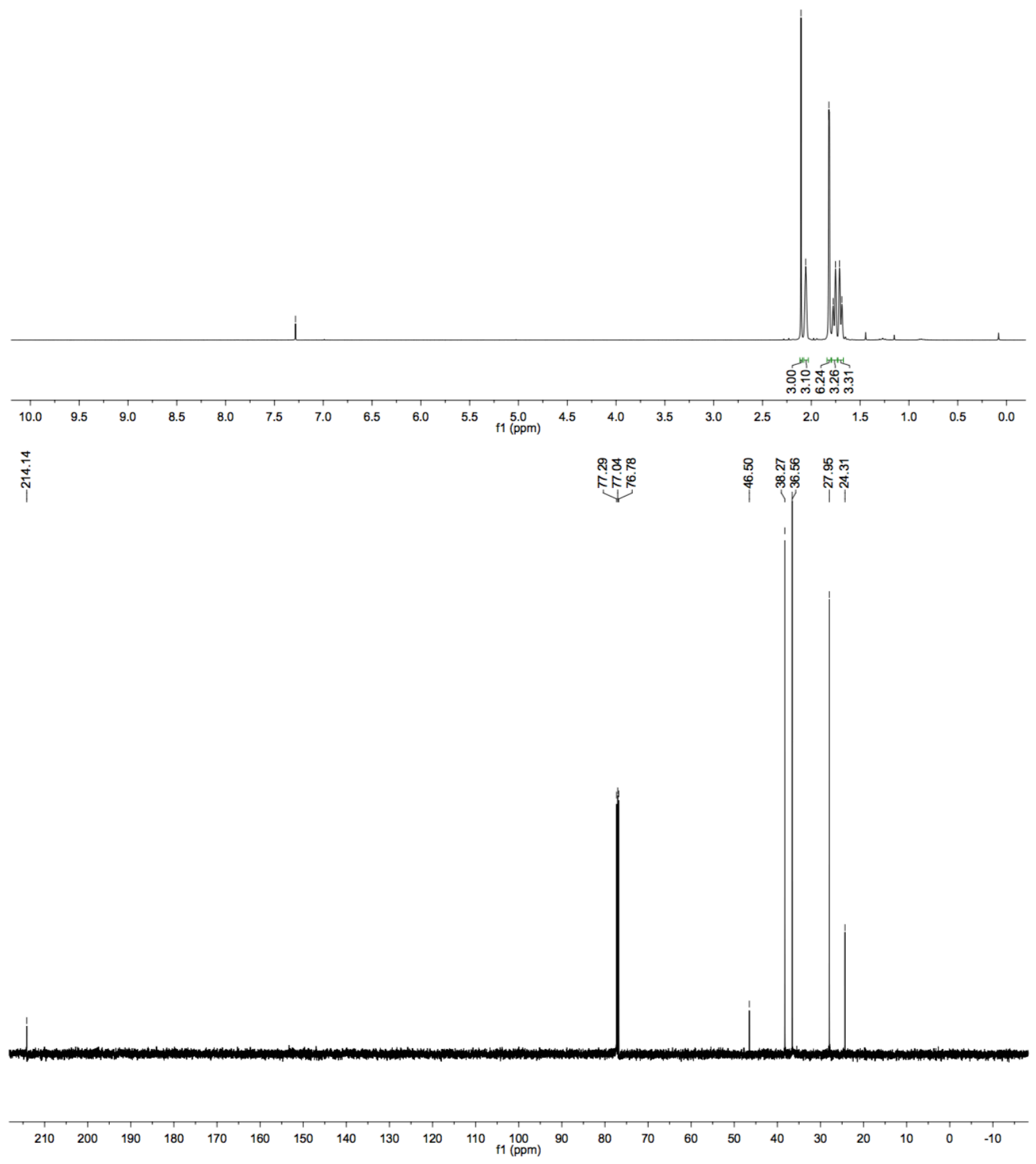

SI-52 


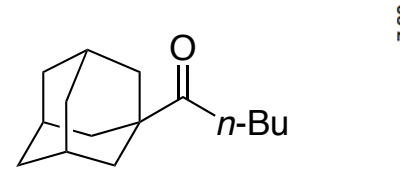

$3 m$
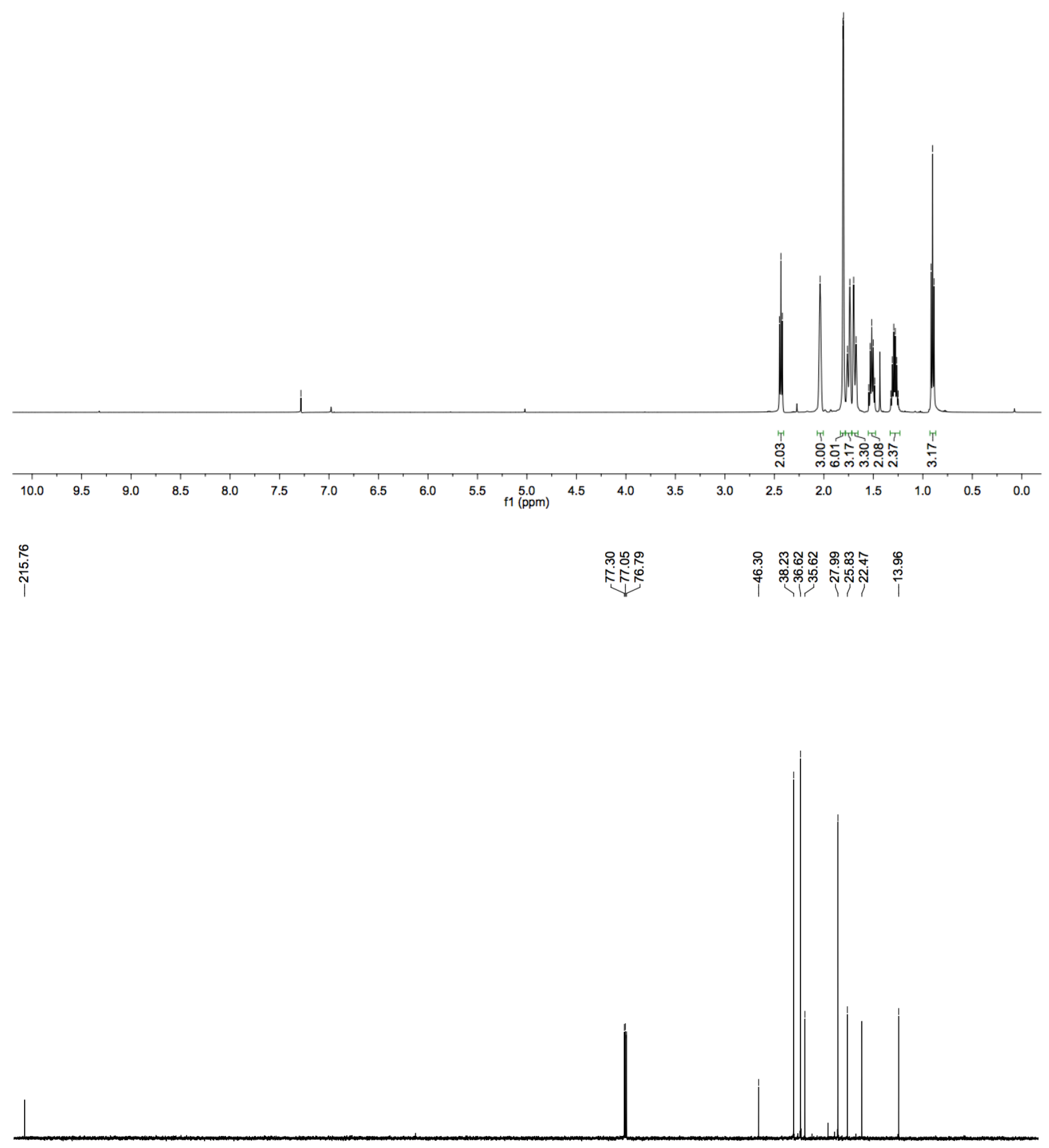

$\begin{array}{lllllllllllllllllllllllll} & 210 & 200 & 190 & 180 & 170 & 160 & 150 & 140 & 130 & 120 & 110 & 100 & 90 & 80 & 70 & 60 & 50 & 40 & 30 & 20 & 10 & 0 & -10\end{array}$

SI-53 

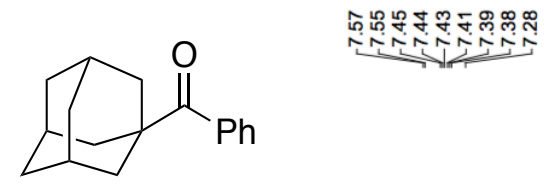

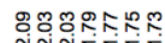

$3 n$

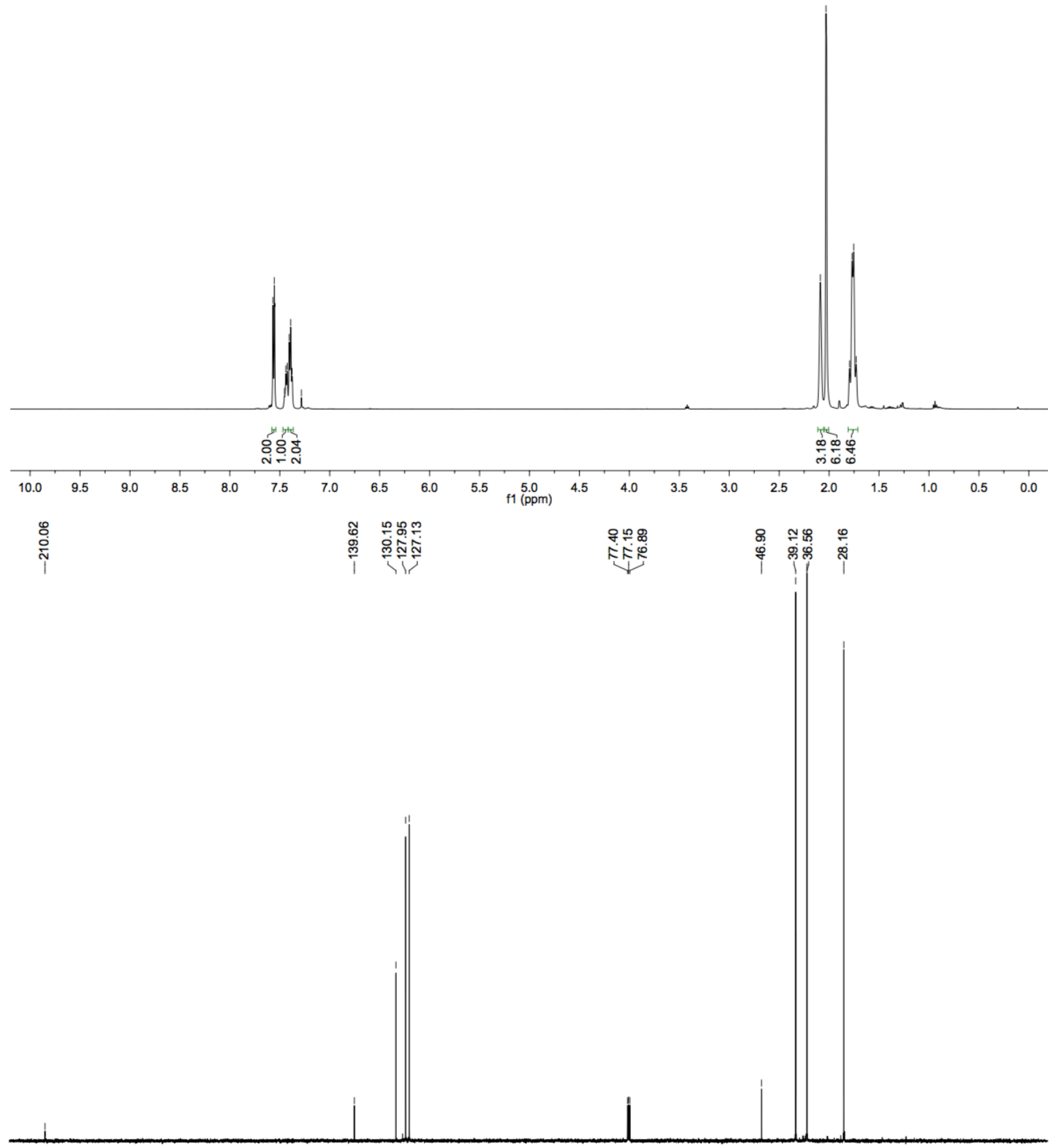

$\begin{array}{llllllllllllllllllllllllllll}210 & 200 & 190 & 180 & 170 & 160 & 150 & 140 & 130 & 120 & 110 & 100 & 90 & 80 & 70 & 60 & 50 & 40 & 30 & 20 & 10 & 0 & -10\end{array}$

SI-54 

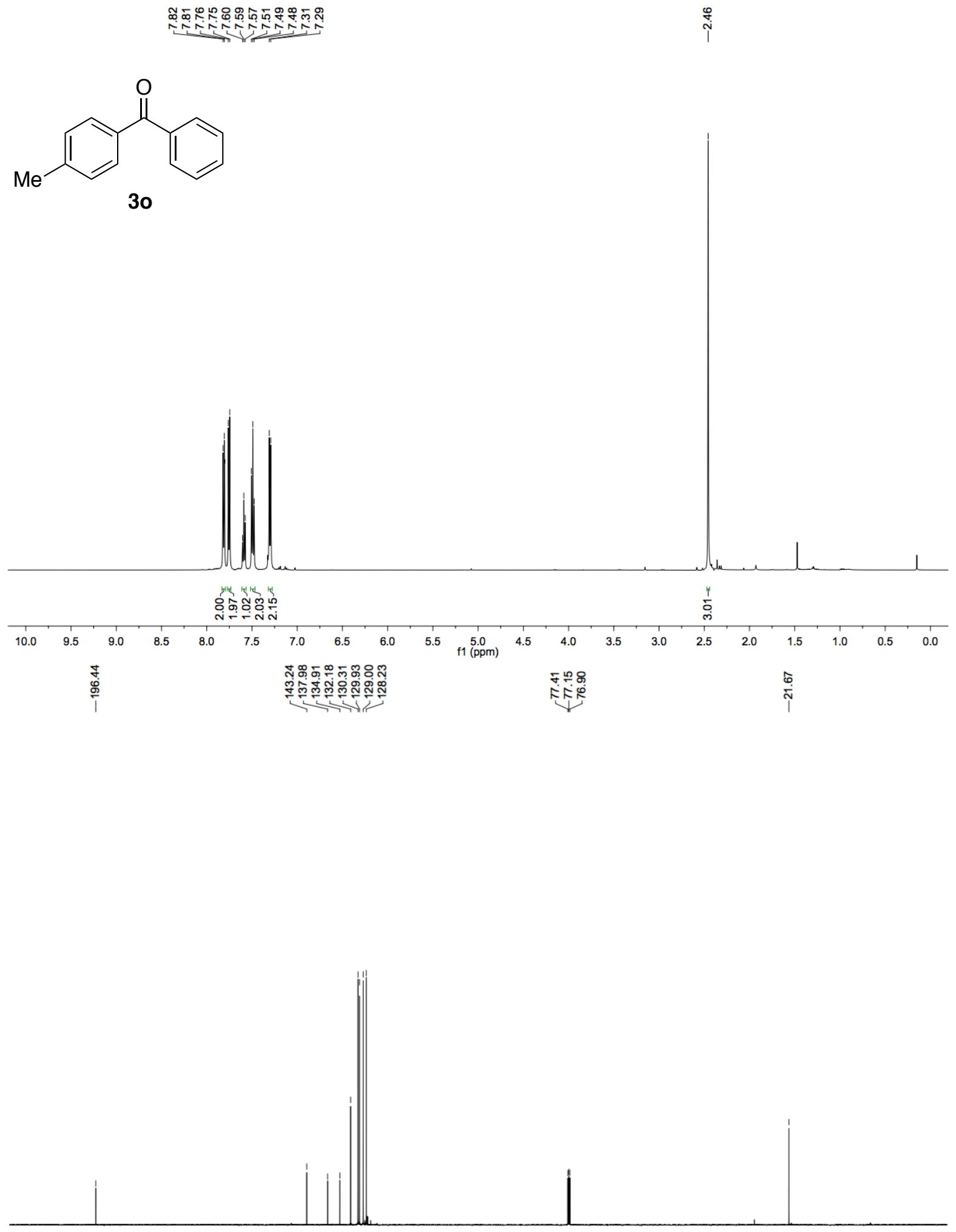

$\begin{array}{llllllllllllllllllllllllllll}210 & 200 & 190 & 180 & 170 & 160 & 150 & 140 & 130 & 120 & 110 & 100 & 90 & 80 & 70 & 60 & 50 & 40 & 30 & 20 & 10 & 0 & -10 & \end{array}$

SI-55 
<smiles>N#Cc1ccc(C(=O)c2ccccc2)cc1</smiles>
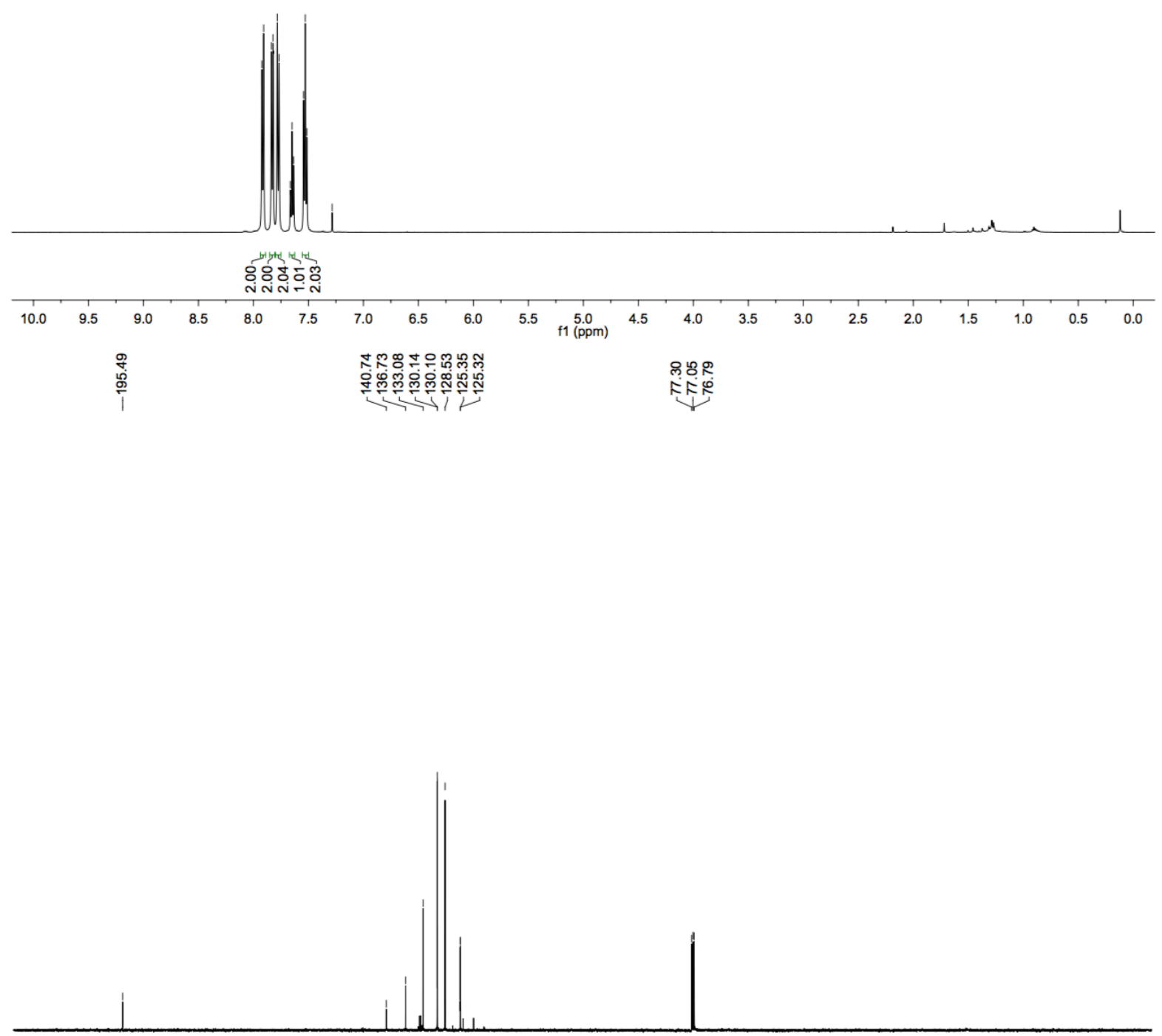

$\begin{array}{lllllllllllllllllllllllllllllll}210 & 200 & 190 & 180 & 170 & 160 & 150 & 140 & 130 & 120 & 110 & 100 & 90 & 80 & 70 & 60 & 50 & 40 & 30 & 20 & 10 & 0 & -10\end{array}$ 
<smiles>O=C(c1ccccc1)c1ccc(C(F)(F)F)cc1</smiles>

$3 p$

$\begin{array}{rrr}1 & 0 & -1 \\ 10 & 0 & -10\end{array}$

$\begin{array}{lllllll}-20 & -30 & -40 & -50 & -60 & -70 & -80\end{array}$

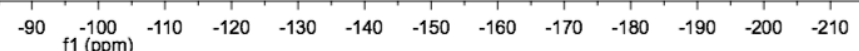



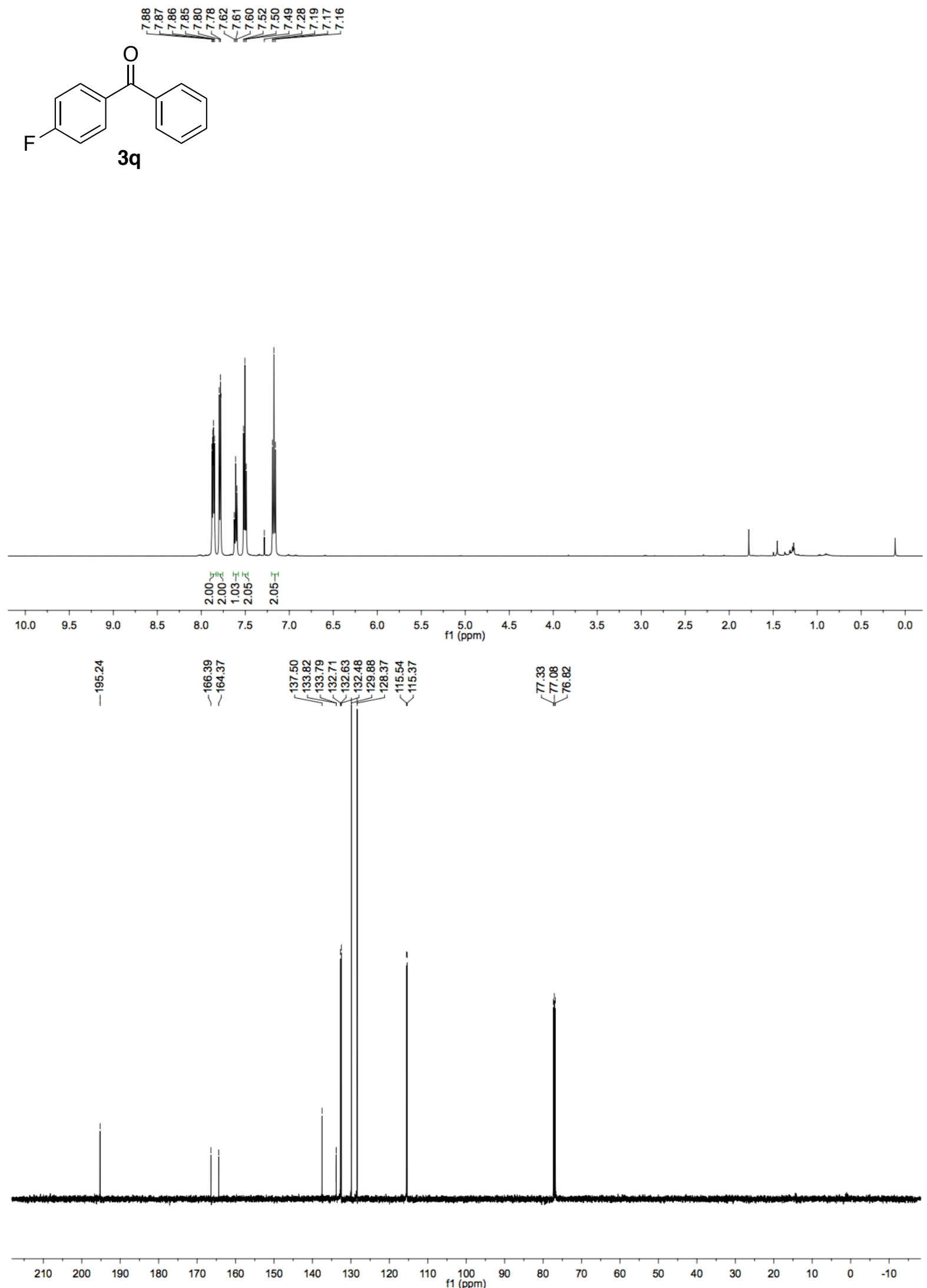

SI-58 
<smiles>O=C(c1ccccc1)c1ccc(F)cc1</smiles>

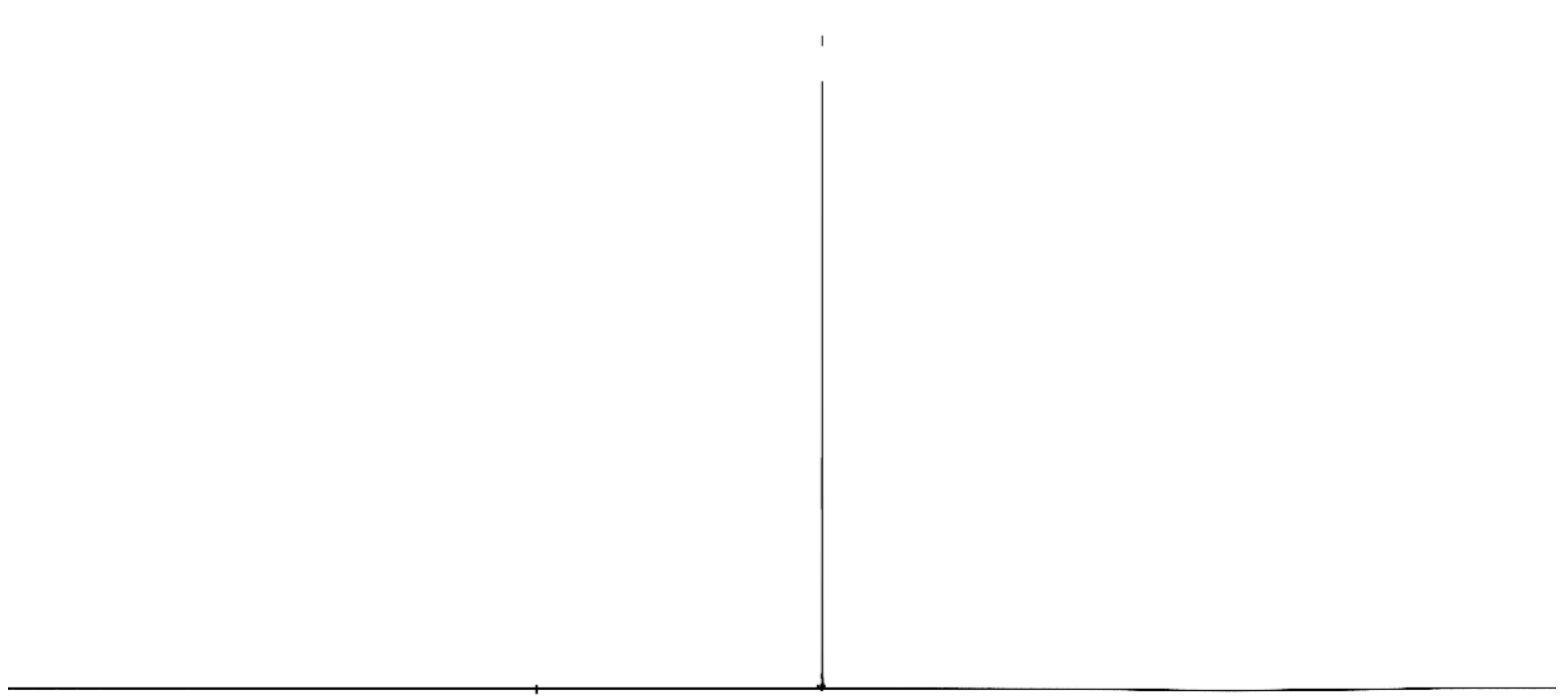

$\begin{array}{llllllllllllllllllllllllll}10 & 0 & -10 & -20 & -30 & -40 & -50 & -60 & -70 & -80 & -90 & -100 & -110 & -120 & -130 & -140 & -150 & -160 & -170 & -180 & -190 & -200 & -210\end{array}$



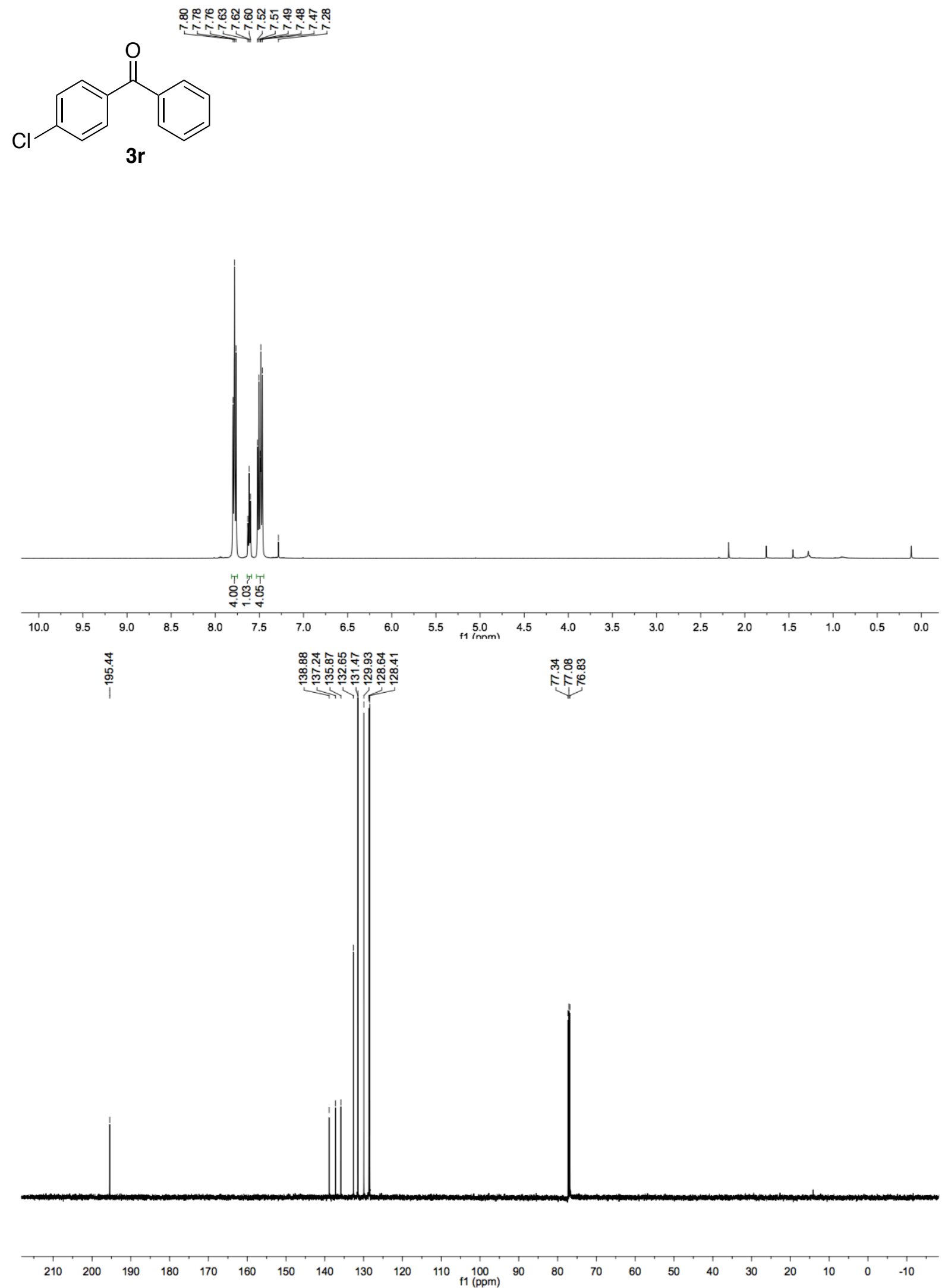

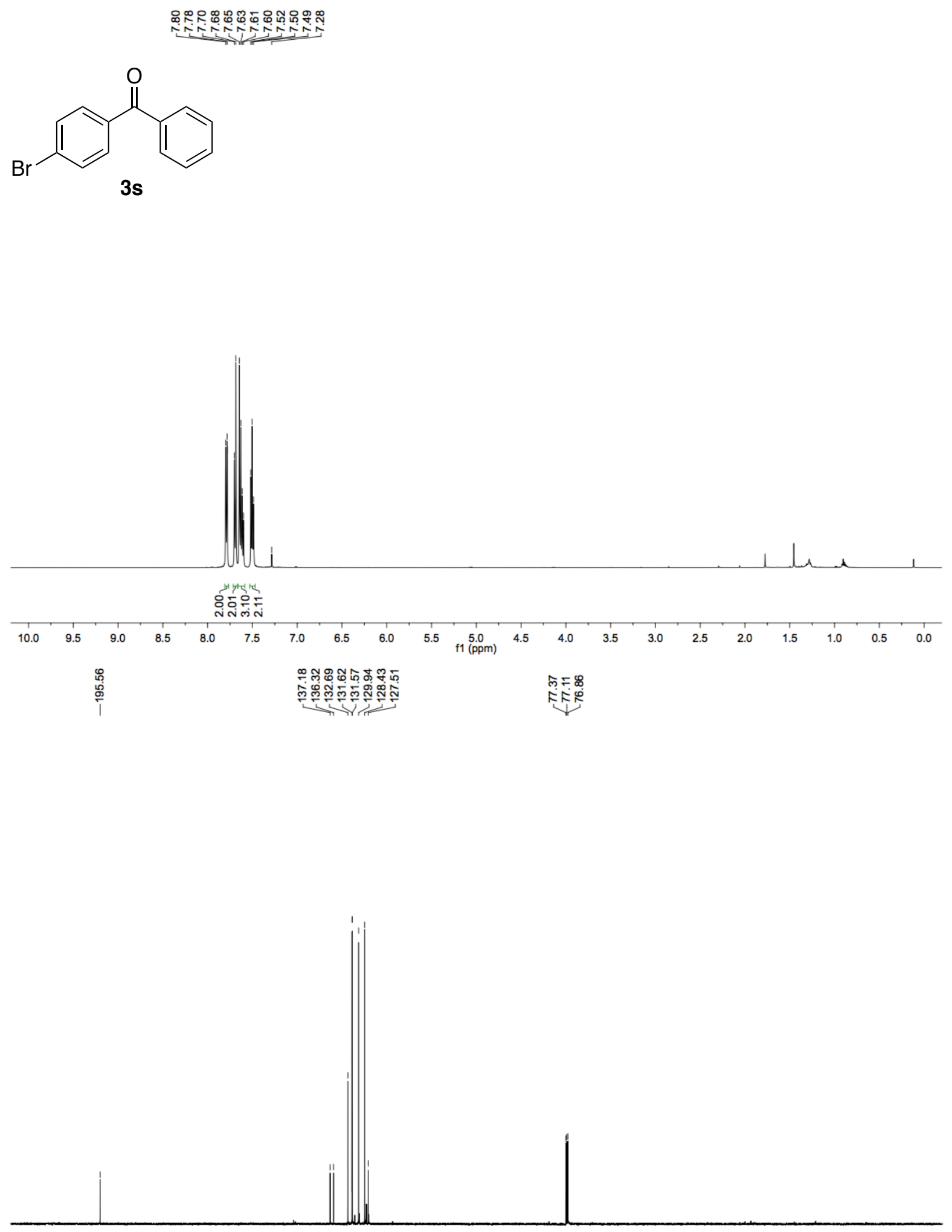

$\begin{array}{llllllllllllllllllllllll}210 & 200 & 190 & 180 & 170 & 160 & 150 & 140 & 130 & 120 & 110 & 100 & 90 & 80 & 70 & 60 & 50 & 40 & 30 & 20 & 10 & 0 & -10\end{array}$ 

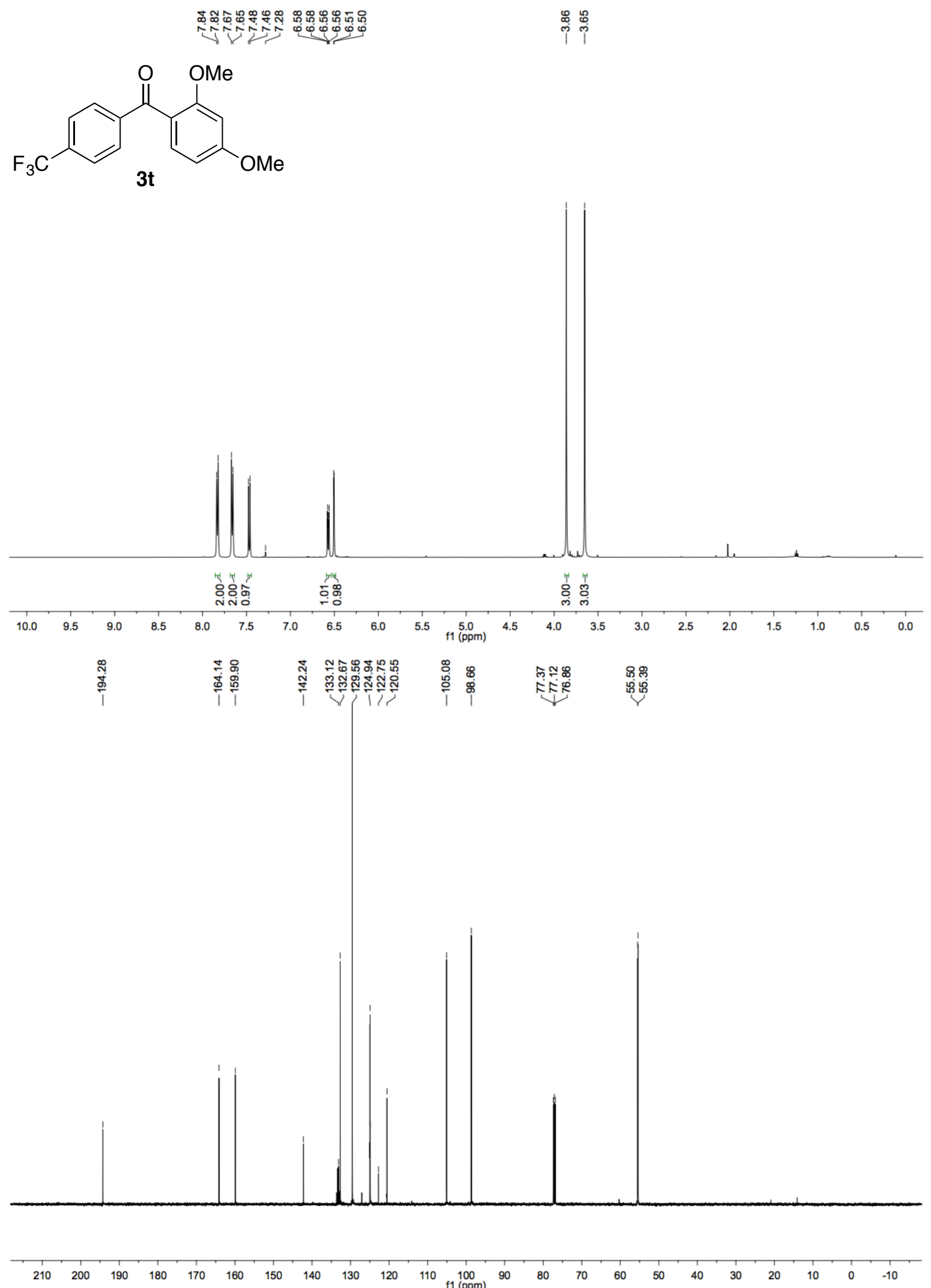


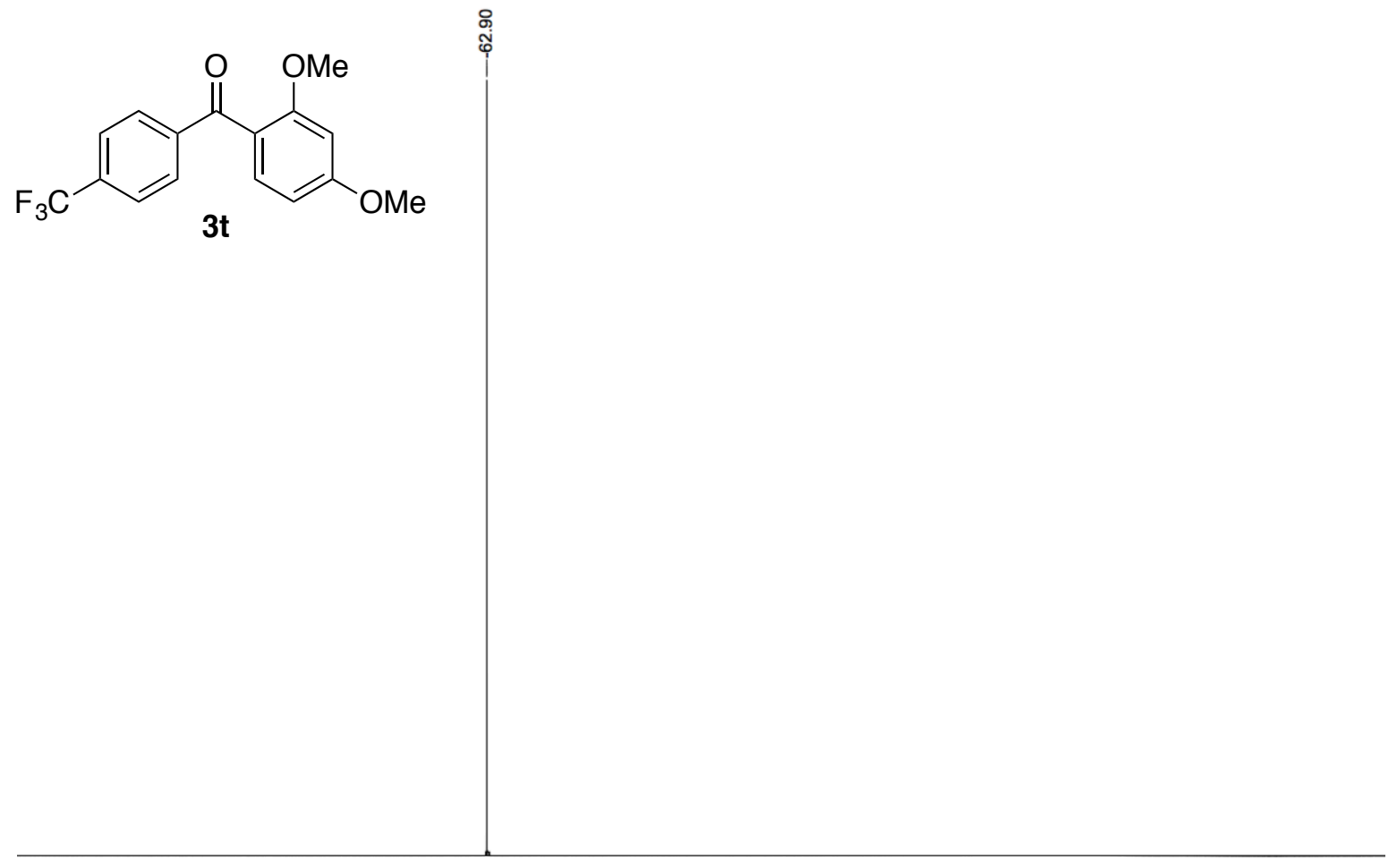

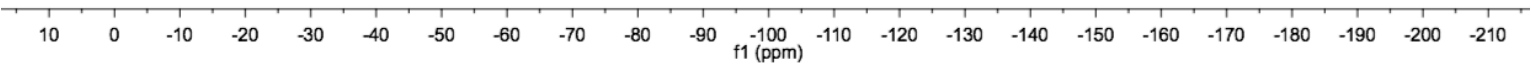



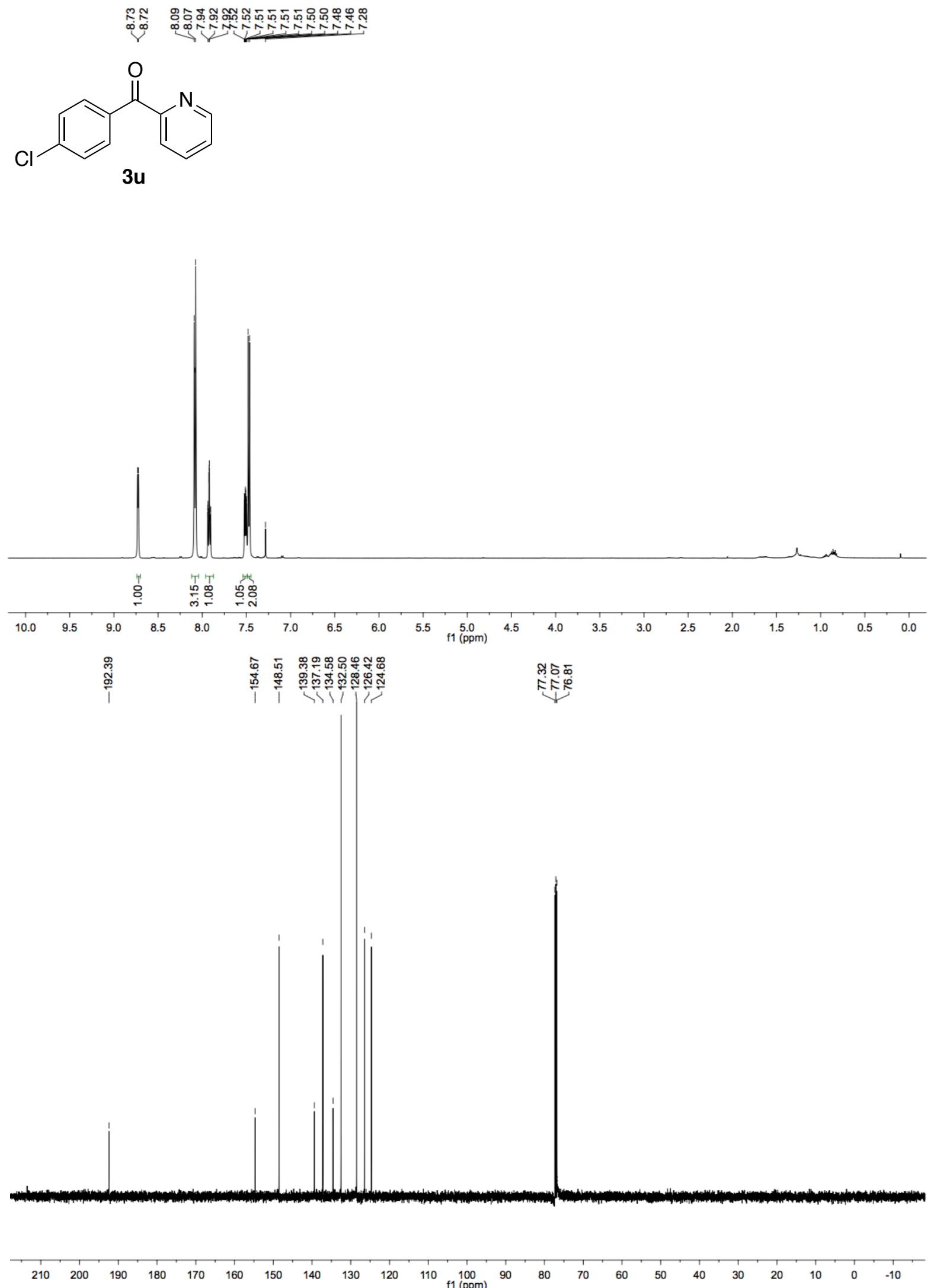
<smiles>Cn1ccc2cc(C(=O)c3ccc(F)cc3)ccc21</smiles>
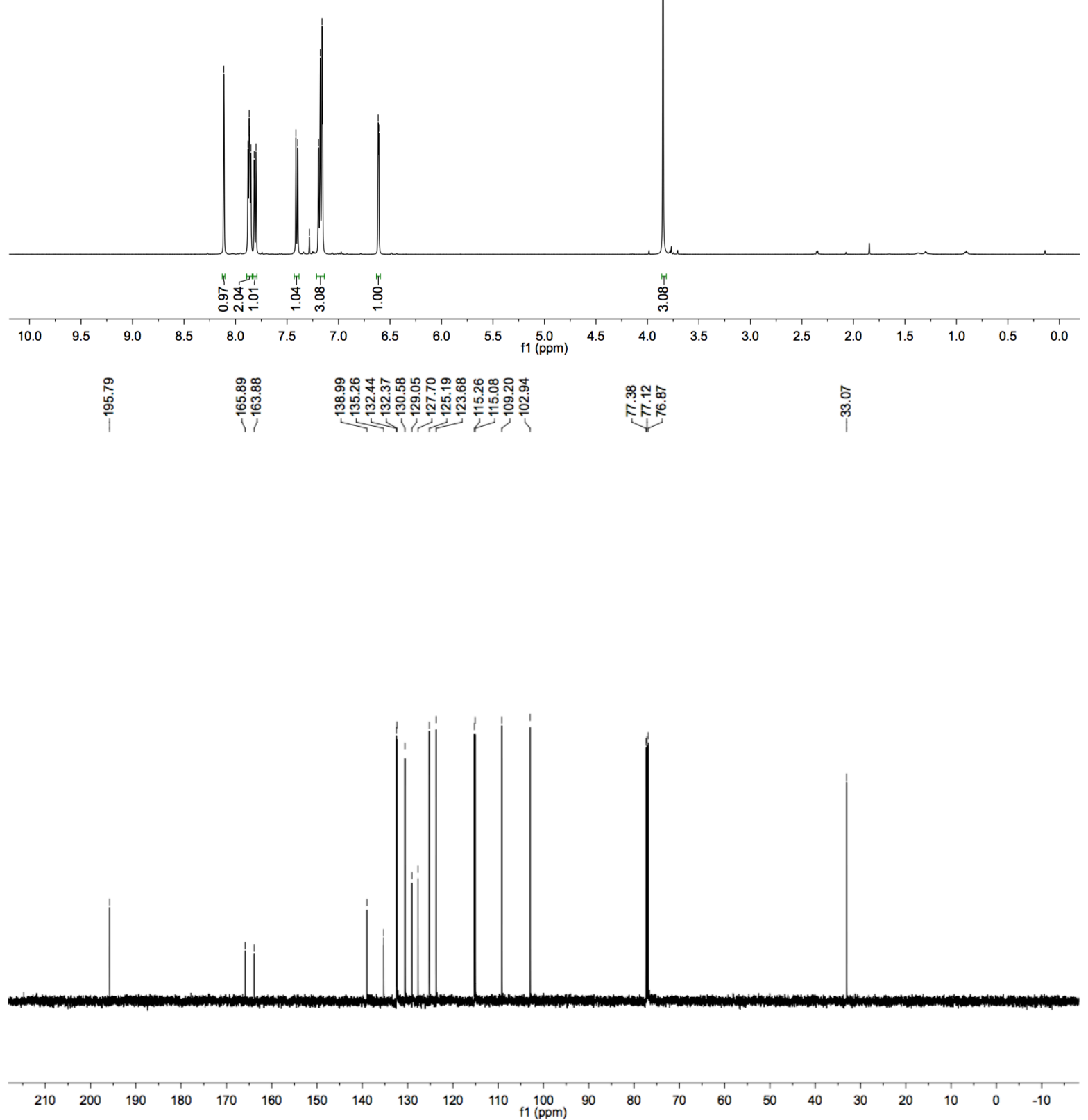
<smiles>Cn1ccc2cc(C(=O)c3ccc(F)cc3)ccc21</smiles>

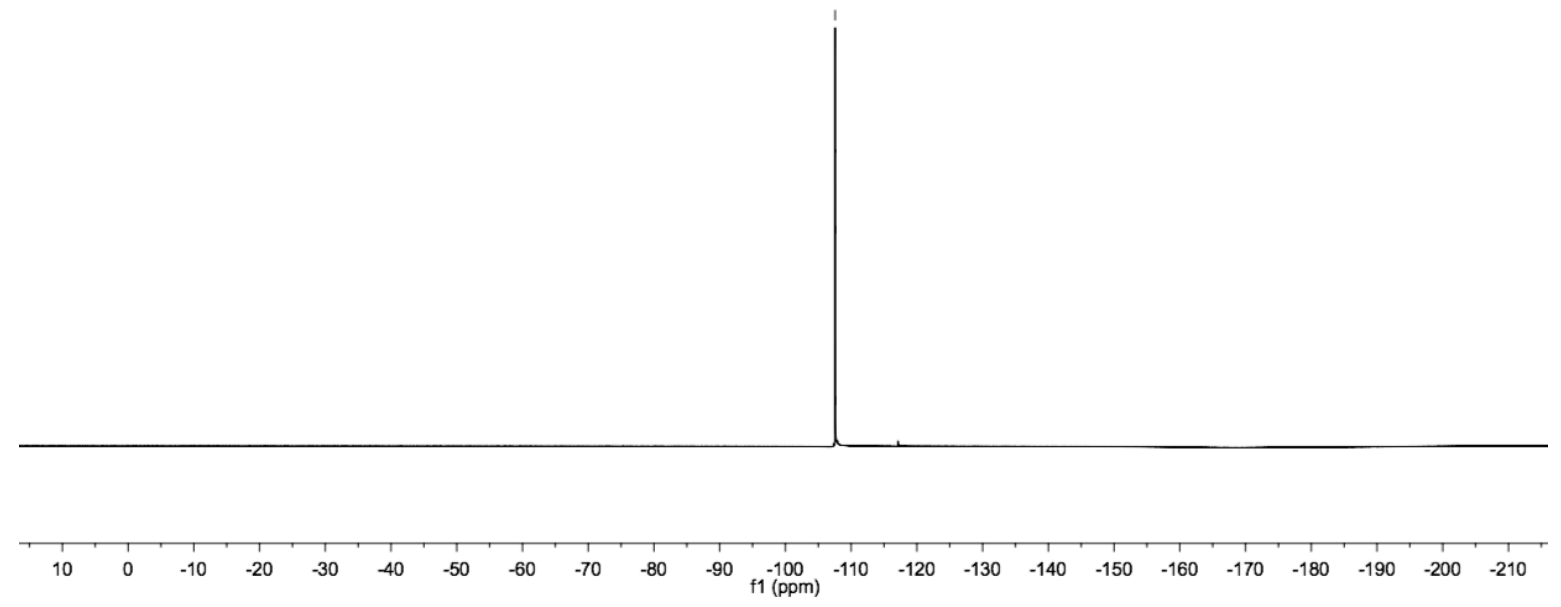



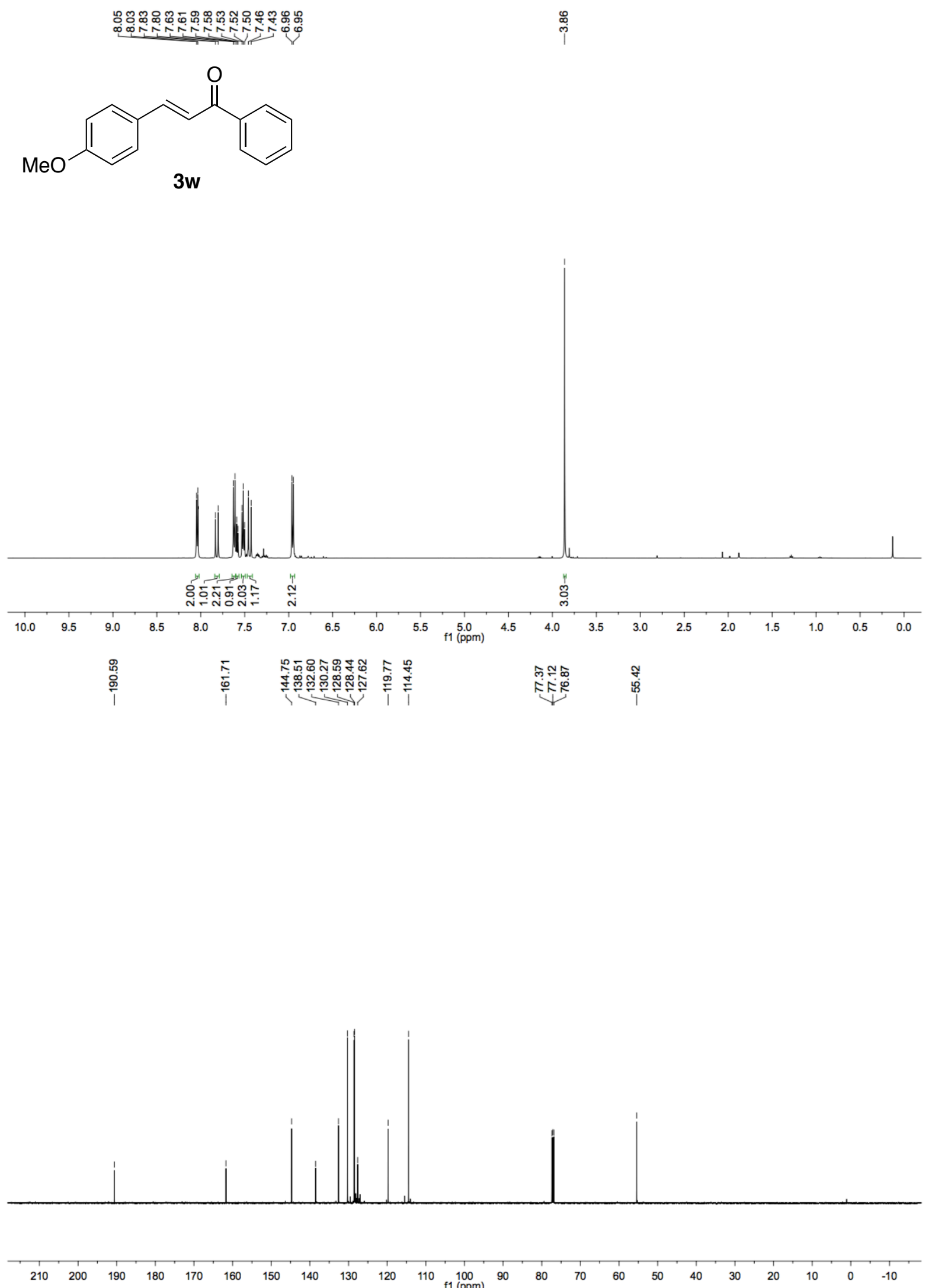